\title{
Evolved health psychology
}

Citation for published version (APA):

Gruijters, S. L. K. (2018). Evolved health psychology: exploring the added value of an evolutionary perspective on health behavior. [Doctoral Thesis, Maastricht University]. Datawyse / Universitaire Pers Maastricht. https://doi.org/10.26481/dis.20180321sg

\section{Document status and date:}

Published: 01/01/2018

DOI:

10.26481/dis.20180321sg

Document Version:

Publisher's PDF, also known as Version of record

\section{Please check the document version of this publication:}

- A submitted manuscript is the version of the article upon submission and before peer-review. There can be important differences between the submitted version and the official published version of record.

People interested in the research are advised to contact the author for the final version of the publication, or visit the DOI to the publisher's website.

- The final author version and the galley proof are versions of the publication after peer review.

- The final published version features the final layout of the paper including the volume, issue and page numbers.

Link to publication

\footnotetext{
General rights rights.

- You may freely distribute the URL identifying the publication in the public portal. please follow below link for the End User Agreement:

www.umlib.nl/taverne-license

Take down policy

If you believe that this document breaches copyright please contact us at:

repository@maastrichtuniversity.nl

providing details and we will investigate your claim.
}

Copyright and moral rights for the publications made accessible in the public portal are retained by the authors and/or other copyright owners and it is a condition of accessing publications that users recognise and abide by the legal requirements associated with these

- Users may download and print one copy of any publication from the public portal for the purpose of private study or research.

- You may not further distribute the material or use it for any profit-making activity or commercial gain

If the publication is distributed under the terms of Article $25 \mathrm{fa}$ of the Dutch Copyright Act, indicated by the "Taverne" license above, 


\section{Evolved health psychology}

Exploring the added value of an evolutionary perspective on health behavior

Stefan L.K. Gruijters 
Colophon

(C) 2017, Stefan L.K. Gruijters, Maastricht, the Netherlands

Cover design by Rachel M. Brown

Printed by Datawyse

ISBN: 9789461598028

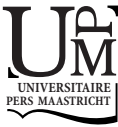

All rights reserved. No parts of this book may be reproduced or transmitted in any form or by any means, without permission from the author or, when appropriate, the publisher of the article. 


\title{
Evolved Health Psychology Exploring the added value of an evolutionary perspective on health behavior
}

\author{
DISSERTATION
}

to obtain the degree of Doctor at Maastricht University, on the authority of the Rector Magnificus, Prof. dr. Rianne M. Letschert, in accordance with the decision of the Board of Deans, to be defended in public

on Wednesday 21 March 2018, at 12:00 hours

by

Stefan L.K. Gruijters 


\section{Promotor:}

Prof. dr. Robert A.C. Ruiter

\section{Copromotors:}

Dr. Karlijn Massar

Dr. Joshua M. Tybur (Vrije Universiteit Amsterdam)

\section{Assessment committee:}

Prof. dr. Gerjo Kok (Chair)

Prof. dr. Abraham P. Buunk (Rijksuniversiteit Groningen)

Prof. dr. Angela D. Bryan (University of Colorado Boulder, USA)

Dr. Ree M. Meertens

Dr. Philippe V.N. Verduyn 


\section{TABLE OF CONTENTS}

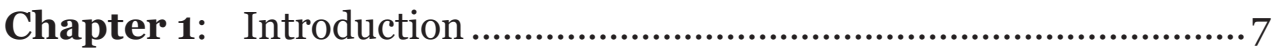

Chapter 2: Function and fitness ...................................................23

Chapter 3: Sex, germs, and health .....................................................43

Chapter 4: Attitude in a motivational context...................................61 61

Chapter 5: What is negative about testing for sexually-transmitted infections? ......................................................................... 77

Chapter 6: General discussion .................................................... 101

References...............................................................................115

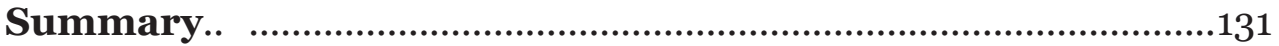

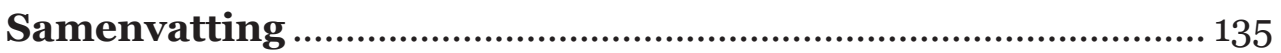

Valorisation ................................................................................... 139

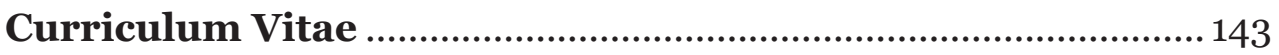

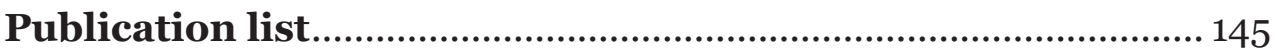

Acknowledgements ................................................................... 149 

Chapter 1: Introduction 

In his book The Selfish Gene, Dawkins (1976) proposed that the abovecited question is likely the first to be posed by a superior species, were they ever to visit our planet and want to assess the level of our civilization. I suggest that a second question would likely concern self-destructive aspects of human behavior. In particular, why does this species - despite their acquired knowledge and rationality - consistently and universally engage in health compromising behaviors?

Examples abound in the general population. Chemicals and toxins are deliberately injected and ingested, and people engage in unsafe sexual practices that lead to infection. For example, a substantial number of people continue to smoke tobacco - shortening average life expectancy by 10 years compared to non-smokers (Jha et al., 2013). Alcohol abuse is still prevalent, and globally a leading cause of morbidity and mortality (Lim et al., 2012). Physical activity levels are not optimal and obesity rates are increasingly high in both children and adults (Lim et al., 2012; see also ten Hoor, Plasqui, Schols, \& Kok, 2014). Globally and annually, 357 million new infections occur through (mostly) unsafe sexual contact (World Health Organization, 2016). More than half of these infections involve bacterial chlamydia (131 million), gonorrhea (78 million) and Syphilis (5.6 million) - all of which, when untreated, can have severe health consequences and impact on quality of life.

For some health damaging behaviors (e.g., substance abuse), understanding the difficulties for people in changing or ceasing these behaviors is perhaps relatively straightforward - the mechanisms of addiction are not part of the 'reasonable brain' but often involve uncontrolled and automatized processes (Houben, Nederkoorn, Wiers, \& Jansen, 2011; Wiers, Rinck, Kordts, Houben, \& Strack, 2010). But for those aspects of health behaviors we have more control over, myriad questions remain. Why do people risk addiction in the first place? And critically, why do people often deliberately and knowingly expose themselves to health risks more broadly?

This question has also occupied health psychologists aiming to understand, predict, and change unhealthy behaviors through research and intervention (Bartholomew Eldregde et al., 2016). A plethora of health behavior theories (HBT) has been developed (e.g., Glanz \& Bishop, 2010) theories that forward an array of variables proposed to causally relate health behaviors. Most HBT are strongly rooted in social-cognitive approaches and focus particularly on cognition, emotion and their interaction with the (social) environment to make predictions about health behavior. Examples of often used theories include the Health Belief Model (e.g., Rosenstock, 1974; Strecher \& Rosenstock, 1997) and the related Protection Motivation Theory (Rogers \& Prentice-Dunn, 1997), Self Determination Theory (Ryan \& Deci, 2000), the Theory of Reasoned Action (Fishbein \& Ajzen, 1975), the Theory of Planned Behavior (Ajzen, 1985), and its recently updated version the Reasoned Action Approach (Fishbein, 2008; Fishbein \& Ajzen, 2010). These 
are only few often used theories amongst the dozens of other theories which are less frequently used in research (cf. Davis, Campbell, Hildon, Hobbs, \& Michie, 2015). Despite the abundant availability of these HBT describing processes through which sociocognitive variables affect behavior, significant challenges and gaps remain.

Some have argued that: 'our attempts at increasing health behavior and decreasing risk behavior may have reached something of a glass ceiling of effectiveness' (Tybur, Bryan, \& Hooper, 2012, p. 862). Indeed, even when behavioral interventions are found to be effective, their effects are usually modest, and persistence of behavior change is variable over time (Davis, Campbell, Hildon, Hobbs, \& Michie, 2015; Michie, Johnston, Francis, Hardeman, \& Eccles, 2008). Meta-analyses (Sheeran et al., 2016; T. L. Webb $\&$ Sheeran, 2006) indicate that key sociocognitive variables, when manipulated, change health behaviors with effect size magnitudes in the small to moderate range (i.e. attitudes $d=.38$, perceived norms $d=.36$, selfefficacy $d=.47$, and intention $d=.38$ ). Put less abstractly, were we to craft an intervention to increase people's position on the strongest of these predictors (self-efficacy), we would (in a best case scenario) see desired behavior change in one individual for roughly every six exposed to this intervention (see Gruijters \& Peters, 2017). This leaves room and need for continued efforts to broaden the underlying theory-base (Michie \& Johnston, 2012) on which health interventions are ideally built to optimize their effectiveness (Bartholomew Eldregde et al., 2016; Davis et al., 2015; Glanz \& Bishop, 2010).

Expansion of the theoretical foundations of HBT may take one of three forms:

1) Integrate existing theories into wider all-encompassing frameworks;

2) Develop and test novel health behavior theory;

3) Shift perspective to a novel level of behavioral analysis.

The first approach is to attempt unification of HBT in integrative frameworks (see Anderson, 1998; Hagger, 2009; Peters \& Crutzen, 2017). Attempts to integrate HBT are likely rooted in the idea that theories capture a small variable set (or part of reality) to predict and explain health behavior (cf. Peters \& Kok, 2016). The argument for integration is that if only all relevant variables were to be charted and their conceptual overlap removed, then HBT could be unified into more effective, efficient, and encompassing theories of health behavior (Hagger, 2009). While there are many examples in the literature of such integrative models (e.g., de Vries, Mesters, Steeg, \& Honing, 2005; Hagger \& Chatzisarantis, 2009), the merits of such theoretical integration is subject of debate (e.g., de Vries, 2017; Gruijters, 2017; Peters \& Crutzen, 2017; Peters \& Kok, 2016; Trafimow, 2017).

A second alternative is to develop and test novel theories of health behavior. Recently it has been remarked that HBT are usually drawn from and restricted to specific disciplines, foremost social psychology, which narrows the possibility to gain advantages of theory from the broader social 
and behavioral sciences (Davis et al., 2015). One approach, these authors suggested, is to widen the scope to other fields and disciplines, including anthropology, sociology, and economics. While such interdisciplinary exploration may benefit development of novel HBT, and may also add to our potential to explain human behavior, yet another tactic may also provide a valuable angle towards this end - a shift of perspective on behavior.

A perspective change may appear practically similar to the development of novel theory. While proposing different relevant variables, HBT can be seen to be unified in the perspective they take on health behaviors - one which we label a process perspective. That is, by describing variables proposed to have causal influence on behaviors (either in additive, interactive, or mediating ways), these theories aim to describe psychological processes generating behavior - and hence are able to make predictions about health behaviors. These variables, then, can be specified and studied at cognitive, (neuro)biological and social-psychological levels (cf. Suls \& Rothman, 2004). Rather than studying 'new' processes, a perspective change may provide a novel layer to theory in health psychology. Evolutionary perspectives, which have recently been gaining momentum in the behavioral sciences, but have thus far seen limited application to questions about health behavior (Tybur et al., 2012), can serve as an alternative. This dissertation explores such an evolutionary perspective on health behavior. Throughout the following chapters, I discuss, illustrate, and test how theory and models rooted in evolutionary science can illuminate what motivates health behaviors. Before proceeding to an overall outline of this dissertation, the sections that follow will first describe the perspective provided by evolutionary theory, how it differs from standard HBT, and how it can complement the field of health psychology.

\section{Defining an 'evolutionary perspective' on behavior}

An evolutionary perspectives can been seen as a meta-perspective (cf. Ronay \& Tybur, 2017). In this sense, 'evolutionary' has been added as an adjective to many existing disciplines, including (evolutionary) biology, (evolutionary) anthropology, and (evolutionary) psychology. Within psychology, the adjective 'evolutionary' has further been added to its subdisciplines, including social psychology (Kenrick, Neuberg, Griskevicius, Becker, \& Schaller, 2010; Neuberg, Kenrick, \& Schaller, 2010), developmental psychology (e.g., Ellis et al., 2012; Frankenhuis \& de Weerth, 2013; Maestripieri \& Roney, 2006), personality psychology (e.g., Buss, 2009; Nettle, 2006; Tybur \& de Vries, 2013), cognitive psychology (e.g., Cosmides \& Tooby, 1996, 2013; Haselton et al., 2009), but recently also health psychology (Tybur et al., 2012). Thus, an evolutionary perspective does not provide alternative, competing, or opposing explanations, but rather aims to complement existing disciplines and sub disciplines in shaping theory and generating novel hypotheses (Mayr, 1961; see also Scott-Phillips, Dickins, \& West, 2011). 
Evolutionary psychology is a modern application of Darwin's evolution theory to the study of human behavior (Cosmides \& Tooby, 2013; Tooby \& Cosmides, 1990). Central is the idea that the human brain and mind, just like other aspects of our biology (e.g. cells, tissues, organs), have gradually and incrementally evolved over vast stretches of time. Evolution is the end result of a simple formula whose elements were partly described by Darwin (1859). The formula requires: 1) variation in traits between individuals, 2) such variation is partly inherited from one generation to the next, and 3) not all variation is equally beneficial to fitness - which broadly refers to variation in individuals' ability to reproduce (Nettle, 2009a; Ridley, 2004). The latter component describes the action of evolutionary selection processes.

In the modern version of evolution theory, the theory is grounded in the field of genetics (e.g., Dawkins, 1976). In this light, evolution is the result of specific gene variants being correlated with fitness enhancing traits, and in case of positive associations the relative frequencies of these variants tend to increase in populations over time (Nettle, 2009a; Ridley, 2004). While important concepts in evolution theory have themselves evolved the last decades (Hamilton, 1964; Kurzban, Burton-Chellew, \& West, 2015), a critical implication of Darwin's original formulation remained steadfast many of our traits and behaviors are not random in evolutionary design, but can be expected to exhibit a functional organization.

In the early 1960s, ethologists and biologists proposed frameworks to capture this implication, and demarcate evolutionary perspectives from the process perspective common in the broader biological, behavioral, and social sciences. Initially a distinction between ultimate and proximate causation was made by Mayr (1961), but the currently dominant framework is based on further subdivisions by Nico Tinbergen (1963; see also, Bateson \& Laland, 2013; Laland, Sterelny, Odling-Smee, Hoppitt, \& Uller, 2011). This framework distinguishes four questions that can be asked about any behavioral trait (see Figure 1).

Two are so-called proximate questions:

1) What are the mechanisms that are responsible for the behavior? (process perspective)

2) What are the developmental processes leading up to these mechanisms? (developmental perspective)

And the two others are ultimate questions:

3) What is the adaptive value of the behavior? (functional perspective)

4) What is the evolutionary history of these mechanisms? (phylogenetic perspective)

Proximate questions (development and process perspectives) are common in HBT; they involve an analysis of individual, group, or social/environmental variables that can help understand the processes underlying behavior. The latter two ultimate explanations can only be meaningfully provided when answers are couched in Darwin's theory of evolution. Evolutionary (phylogenetic) explanations, however, are usually 
not central to the work of evolutionary psychologists, who instead focus on adaptive value of behavior, and test functional hypotheses about behavior. Box 1 illustrates the various explanatory levels using obesity as an example.

Box 1. An illustration of the ultimate-proximate distinction.

\section{Proximate / mechanism (what are the mechanisms causing the behavior?)}

Consider the example of a common health problem - obesity - which has been associated with dysregulated eating patterns (e.g., eating when not hungry). Addressing the first proximate question involves testing hypotheses about potential mechanisms leading to obesity, and one such implicated mechanism is impulsivity (e.g., Houben, Nederkoorn, \& Jansen, 2014; Nederkoorn, Smulders, Havermans, Roefs, \& Jansen, 2006) - a mechanism that may partially explain dysregulated eating patterns.

\section{Proximate / development (how do the mechanisms develop in an individual?)}

Another type of proximate explanation for impulsive eating can be given from a developmental perspective, either by examining genetic aspects of individual differences in impulsivity directly (e.g., Anokhin, Grant, Mulligan, \& Heath, 2015), or by looking at how such genetic variation unfolds during development in interaction with environments (see Champagne \& Mashoodh, 2009). To illustrate this, recent research has shown that growing up in stressful, unpredictable environments, is related to dysregulated eating patterns because children who are exposed to such circumstances tend to have higher levels of impulsivity (Maner et al., 2017). Early environments' calibration of impulsivity mechanisms, in interaction with genetic correlates of such a mechanism, is an example of a behavioral explanation provided from a developmental level of analysis.

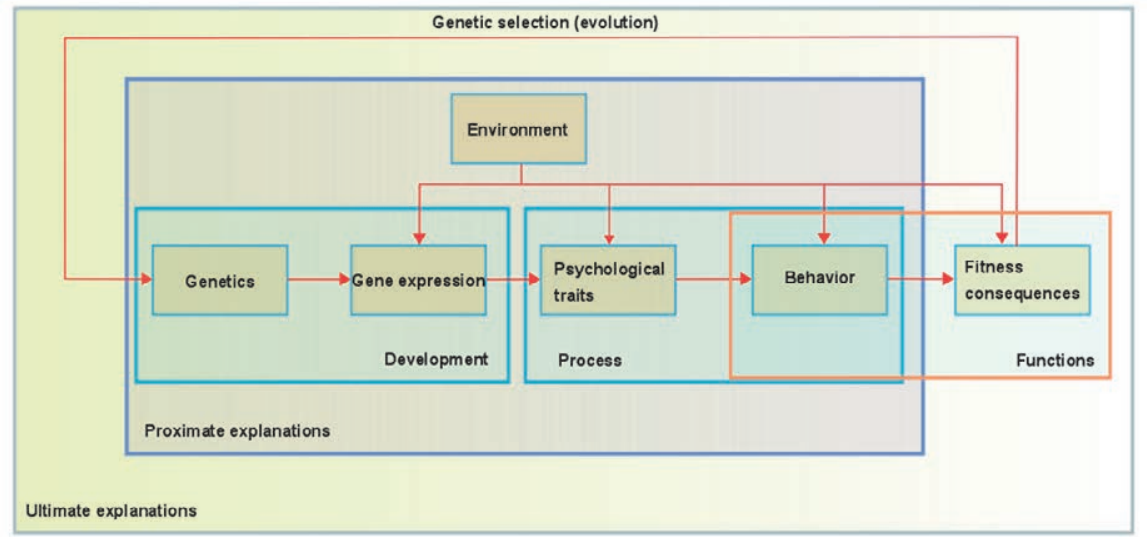

Figure 1. Ultimate and Proximate perspectives on behavior

Note. The illustration is based on Mayr (1959), Tinbergen (1961), and is partly adapted from Laland et al. (2011). 


\section{Box 1. (Continued...)}

\section{Ultimate / function (what is the adaptive value of the behavior?)}

Functions of psychological mechanisms can be given without reference to reproductive fitness (e.g., how behavior contributes to achieving some defined goal). Evolutionary scientists however define functions in terms of how behavior affects reproductive fitness - the adaptive value of behavior. One notion about the adaptive value of impulsive eating involves the function of fat storage as a buffer against future shortage (see also Ahlstrom, Dinh, Haselton, \& Tomiyama, 2017). A recently posited hypothesis - the Insurance Hypothesis (Nettle, Andrews, \& Bateson, 2016) predicts that if such insurance is indeed the adaptive value of overeating, then we might expect individuals to do so especially when they receive cues that food access may be insecure; a functional hypothesis that can be empirically examined (Nettle et al., 2016).

\section{Ultimate / phylogeny (what is the evolutionary origin of the behavior?)}

Finally, applying the fourth explanation for behavior involves unravelling how the mechanisms evolved from ancestral forms- in human's Paleolithic ancestors, and before - to the present (see Tooby \& Cosmides, 1990). It answers the question what historical selection events led to the evolution of species-typical mechanisms (e.g. impulsivity/reward systems). Phylogenetic questions on the origins of psychological mechanisms are pursued by for instance comparative psychologists and evolutionary anthropologists (see Nunn \& Barton, 2001) and can also test hypotheses about evolved functions of traits - but are not central to the work of evolutionary psychologists.

When applying the evolutionary question of adaptive value to various health damaging behaviors, we are faced with an apparent paradox. What is adaptive about self-damaging behaviors such as smoking? Or health risk taking behaviors such as engaging in unprotected sex? The discussion of the Mayr-Tinbergen framework helps to elucidate that concepts such as adaptive versus maladaptive behaviors take on a very different meaning once examined from an evolutionary perspective (see also, Ellis et al., 2012). From a non-evolutionary perspective, definitions of what kind of behavior is adaptive or maladaptive are usually given in terms of their costs on physical safety, well-being and health, for both the individual as the (social) environment. In this sense, it is tempting to view most risky health behaviors (e.g., drinking, smoking, unsafe sex) as maladaptive, costly behaviors.

An evolutionary perspective applies a different analysis of the costs and benefits of behaviors - one that trades in reproductive fitness as its main currency. Evolutionary theory, then, predicts that human psychology evolved mechanisms that optimize reproductive fitness, but that such optimization involves tradeoffs in the costs and benefits of behaviors. Seen 
from this perspective, many risk-taking behaviors may a have certain probability of causing health issues later in time (delayed fitness costs), but may more directly promote reproductive fitness in the present (direct fitness benefits). For example, the various risk taking behaviors performed by adolescents and young adults may ultimately have fitness benefits - if, for instance, such behaviors increase individuals' status and reputation in social groups (see Ellis et al., 2012). An evolutionary perspective, then, predicts that human psychology evolved computational mechanisms that optimize such tradeoffs in generating behaviors (see also Tybur et al., 2012).

Once concepts such as adaptive versus maladaptive, or costs versus benefits, are redefined in reproductive fitness currency, we get a different picture on how costly risky behaviors are to individuals. Viewed from an evolutionary perspective, and evaluated in terms of fitness effects, many health compromising behaviors are not necessarily fitness compromising. Accordingly, given that evolutionary theory predicts traits and behavior to optimize fitness, it thus also predicts that health may not necessarily be a prioritized goal people pursue. Chapter 2 pursues this idea, and reviews theory and empirical work relating to the discrepancy between the concepts fitness and health. The question, then, is what can be expected to motivate health-related behavior - if not health as an ultimate goal per se? The next section discusses some tenets of an evolutionary perspective on healthprotective and health promoting motivations.

\section{Problems of the past explain the motives of the present}

Several evolutionary psychological frameworks of motivation propose that specific motives likely evolved as part of human's species-typical psychology (Aunger \& Curtis, 2013; Bernard, Mills, Swenson, \& Walsh, 2005; Kenrick, Griskevicius, Neuberg, \& Schaller, 2010; Mark Schaller, Kenrick, Neel, \& Neuberg, 2017). Individual motives can be understood in these frameworks as 'psychological mechanisms designed by evolution to cause animals to seek to meet a need through behavior' (Aunger \& Curtis, 2013, p. 51).

At the core of this perspective is the principle of functional modularity (e.g., Barrett, 2012; Barrett \& Kurzban, 2006): the view that the human mind likely evolved domain-specific computational mechanisms, or 'contentrich adaptive problem-solving systems' (Cosmides \& Tooby, 2013, p. 202). These evolved over time because they helped human ancestors solve specific (e.g., avoiding infection), rather than general problems (e.g., avoiding death). For instance, the motivational systems involved in self-protection are likely distinct from those dealing with disease threat (Neuberg, Kenrick, \& Schaller, 2011): These systems respond to different cues (e.g., angry faces versus rashes), utilize different emotions (e.g., fear versus disgust), and result in different action patterns (e.g., escaping versus cleansing).

The notion of domain-specificity can be contrasted with assumptions underlying belief-centered HBT - such as the Health Belief Model (Janz \& 
Becker, 1984), Protection Motivation Theory (Rogers \& Prentice-Dunn, 1997), and the Reasoned Action Approach. That is, belief-centered models carry an assumption of domain-generality - as long as a potential threat is sufficiently recognized as such, and given people's ability to neutralize the threat, then these models predict health-protective motivation regardless of the nature of the threat. In this sense, these models do not make theoretical distinctions between the sorts of threat that is appraised. Rather, neutralizing the psychological consequences (e.g., anxiety) of any threat is seen as the ultimate motive for behavior (cf. Holbrook, 2016).

For example, in the Reasoned Action Approach, expectancy-value theory is central to understand the formation of attitudes (e.g., Ajzen \& Fishbein, 2008). Expectancy-value theory proposes that motivation is partly the result from an evaluation of the consequences (how likely a behavior is to have a specific consequence) and how desirable such an outcome of behavior would be. Protection Motivation Theory (Rogers \& Prentice-Dunn, 1997), as a second example, targets more specific beliefs about the consequences of behavior - grouped under threat appraisals and coping appraisals. Threat appraisal is a function of individuals' beliefs about their susceptibility and the seriousness of the health danger. Coping appraisal is determined by beliefs about how effective a response to the danger would be, and whether an individual has the ability to effectively respond to the threat (see also Floyd, Prentice-Dunn, \& Rogers, 2000; Peters, Ruiter, \& Kok, 2013). Overall, these models do not make theoretical distinctions between different sorts of threats, and the motivation to engage in health-protective behavior is considered to be a function of the various beliefs relating to a threat and the protective action.

By contrast, evolutionary models of motivation predict instead that different sorts of threats have resulted in domain-specific motivational systems - and thus these models are, in a way, threat-centered. Shared among evolutionary models of motivation is that each model begins with analyzing the sorts of recurrent problems our ancestors faced in the past to then make predictions about solutions (in the form of psychological mechanisms) likely to be favored by selection processes. The fundamental motives framework (Kenrick, Griskevicius, et al., 2010; Kenrick, Neuberg, et al., 2010; Mark Schaller et al., 2017) proposes a flexible hierarchy of functionally distinct motivational systems likely to have evolved in human psychology - including systems involved in regulating parenting, mate retention, mate acquisition, status/esteem, affiliation, self-protection, and immediate physiological needs. Each of these are thought to have distinct motivational systems (including various emotions) managing the threats and affordances within these domains.

For instance, threats to mate retention result in the emotion jealousy (e.g., Massar \& Buunk, 2009), and activation of a mate guarding goal-state shifts attention to domain-relevant aspects of the environment (e.g., rivals; see also Maner, Gailliot, Rouby, \& Miller, 2007). Alternatively, threats to the 
maintenance of group integrity (e.g., cheaters, non-cooperators) involve feelings such as moral disgust and contempt (Aunger \& Curtis, 2013; Tybur, Lieberman, Kurzban, \& DeScioli, 2013). Additionally, work suggests that threat-cues pertaining to non-cooperative, untrustworthy people involve attention biases (Vanneste, Verplaetse, Verhiel, \& Braeckman, 2007), boost source memory performance (Buchner, Bell, Mehl, \& Musch, 2009), and improve conditional reasoning (Cosmides, 1989; Van Lier, Revlin, \& De Neys, 2013).

In sum, evolutionary models of motivation assume that not all threats are equal. Instead, our evolved psychology has been shaped to prioritize cues to specific threats that have had consequences on reproductive fitness in ancestral environments. Thus, such a view implies that a focus on these domain-specific, evolved, motivations may effectively increase our understanding of health behavior. One such hypothesized domain-specific motivational system has been proposed to be particularly relevant to health behavior questions (Tybur et al., 2012), and is central to this dissertation: Pathogen-avoidance motivation.

\section{The evolved psychology of pathogen-avoidance motivation}

Throughout evolution, pathogens (e.g., viruses, bacteria, parasites, fungi) have posed selective pressures on ancestral populations (W. D. Hamilton, Axelrod, \& Tanese, 1990). The consequences of pathogens have also led to the evolution of a complex system of defenses - such as the innate and adaptive immune systems (e.g., Cooper \& Alder, 2006). In addition to such physiological adaptations, scholars have proposed that humans evolved another line of defense against pathogens which is managed behaviorally - some have labeled this a 'behavioral immune system' (Mark Schaller \& Duncan, 2007; Mark Schaller \& Park, 2011), but I will use pathogen-avoidance motivation to refer to this system.

Arousal of pathogen-avoidance motivation has specifically been linked to the emotion disgust in the literature (Curtis, de Barra, \& Aunger, 2011; Oaten, Stevenson, \& Case, 2009; Tybur \& Lieberman, 2016; Tybur, Lieberman, \& Griskevicius, 2009; Tybur et al., 2013). This idea is bolstered by investigations showing that many disgust elicitors objectively correlate with increased probability of pathogen infection. Consider disgust elicitors such as 'vomit', 'feces', 'blood', 'urine', 'saliva', 'rotten foods', which share that all of these are objectively linked to a high risk of containing pathogens (Curtis, 2013). This link suggests a clear adaptive function for disgust to regulate avoidance of pathogens. For this reason, disgust responses elicited by pathogen cues have specifically been labeled as pathogen disgust (Tybur et al., 2013). These pathogen-avoidance motivational systems are perhaps one specialized - but foundational - correlate of our evolved health motivation.

Since pathogenic microbes are too small to detect directly (unlike other larger sized threats), attentional and perceptual mechanisms are predicted 
to be attuned to various cues that connote pathogen presence. Such cues can be gustatory, olfactory, or visual (e.g., specific tastes, smells, and colorings which cue pathogen presence in foods), and they trigger pathogen disgust and precautionary action (e.g. casting food from the mouth or distancing from the source). The psychology of pathogen-avoidance thus involves a set of diagnostic tools to detect pathogens via perceptual cues of their presence. The relationship between cues and actual presence (or perceived state and actual state), however, is statistical as opposed to logical (see also Mark Schaller \& Park, 2011; Tybur \& Lieberman, 2016; Tybur et al., 2013). For example, some rashes may reliably indicate the presence of fungi or viruses, whereas others may result from autoimmunity and thus do not cue pathogen presence. Additionally, some tastes (e.g. fermentation related odors) may trigger disgust but the pathogens may not necessarily be harmful. Thus, similar to any other perceptual detection task under conditions of noise (e.g. diagnostic scans and screening for diseases), there are issues of sensitivity and specificity at stake. While natural selection is expected to have shaped a pathogen-avoidance motivation with - on average - high sensitivity rather than specificity (cf. Nesse, 2005), the systems are likely fine-tuned differently between individuals (Tybur \& Lieberman, 2016).

Individual differences in pathogen-avoidance (often measured with trait measures of pathogen-disgust sensitivity (see Tybur, Frankenhuis, \& Pollet, 2014)) have been linked to various outcomes, including sexual behavior (Duncan, Schaller, \& Park, 2009), political attitudes and ideology (Tybur et al., 2016; Tybur, Inbar, Güler, \& Molho, 2015; Tybur, Merriman, Hooper, McDonald, \& Navarrete, 2010), stigma towards obese individuals (Lieberman, Tybur, \& Latner, 2012; Park, Schaller, \& Crandall, 2007), and ethnocentrism (Navarrete \& Fessler, 2006). At first blush, this appears to be a disconnected array of empirical findings without a clear organizing principle to be found. Yet, these various findings have been proposed to relate to the functioning of our evolved pathogen-avoidance mechanisms. The hypothesized organizing principle in these findings is a better safe than sorry' set-up of the pathogen detection systems - signs of abnormality/unfamiliarity are more likely to be perceived as a pathogenthreat for individuals positioned high in such motivation. While pathogenavoidance motivation has been linked to various interpersonal behaviors and attitudes, limited work has related this motivation to individual differences in health-protective behaviors. Chapter $\mathbf{3}$ discusses and examines the role of individual differences in this motivation and its relation with various screening attitudes and intentions.

Individual differences can be understood as stable (trait level) variation in the psychological mechanisms involved in either pathogen-detection systems or in the subsequent emotional and cognitive processing of pathogen cues (Tybur \& Lieberman, 2016). But such motivations have also been shown to be state and context dependent. For example, Tybur, Bryan, Magnan, and Hooper (Tybur, Bryan, Magnan, \& Hooper, 2011) sprayed a lab 
with an odor similar to those associated with pathogen sources. These researchers hypothesized that this olfactory cue to pathogens would motivate health-protective action - that is, the cue was expected to increase participants' intention to use, buy, and discuss the use of condoms in future sexual encounters. Results supported their hypothesis; participants in the pathogen cue condition reported greater condom use intentions than control group participants. Chapter 4 proceeds to examine such state-activation of pathogen-avoidance motivation in relation to screening attitudes and how such an induced motivation influences the relevance of specific beliefs held about screening.

Finally, as described previously, pathogen-avoidance motivation has also been important to understand interpersonal attitudes and behaviors (e.g., Kurzban \& Leary, 2001; Tybur et al., 2016). Other people are a primary source of pathogens, with potentially detrimental consequences on fitness. As a solution to such an adaptive problem, avoidance of any form of interpersonal interaction would be an adequate precautionary strategy - but avoiding the threats of sociality would also mean losing the benefits of maintaining close social ties (Neuberg et al., 2011). Thus, there is a tradeoff involved in the costs and benefits of sociality and interpersonal interactions. Sexual behavior can provide a concrete illustration of such a tradeoff - while engaging in casual sex can be beneficial to individuals' reproductive fitness, it also simultaneously increases the risk of pathogen infection. Such threats and affordances of social interaction are expected to be navigated differently by people. Thus, pathogen-avoidance motivation - in a sense - may provide insights into the psychological distance people put between themselves and others. More generally, it has been proposed that pathogen-avoidance may be a critical motivational factor underlying avoidance of others that are perceived to be of high infection risk - and this function has been suggested to illuminate stigmatization (Kurzban \& Leary, 2001; Neuberg, Smith, \& Asher, 2000). Chapter 5 extends this idea in relation to perceived barriers (such as anticipated negative responses) by people who get screened for infectious disease - and aims to find out whether pathogen-avoidance motivation has a role in understanding negative attitudes towards the 'potentially' infected - as yet another possible health-protective function of pathogen-avoidance motivation.

\section{Overview of this dissertation}

The main focus of this dissertation is to explore how an evolutionary perspective on motivation can increase our understanding of health behavior. I do so by applying this perspective foremost to a specific health behavior - screening for sexually-transmitted infections (STI). Infectious disease has posed severe challenges to health and well-being in the past, and continues to do so in the present. Despite the many efforts to promote safe sexual practices (see Scott-Sheldon, Huedo-Medina, Warren, Johnson, $\&$ Carey, 2011), STI are still prevalent and a source of health problems 
(World Health Organization, 2016). Available treatments for STI (either curative or palliative) can have no effect when people remain undiagnosed therefore, actively seeking health care services and getting screened are critical steps needed to be taken by individuals. This raises a need to further understand the motivations that drive people towards health protective behaviors.

Chapter 2 first reviews the evolutionary psychological literature, and discusses what an evolutionary perspective can offer health psychology. The chapter sets-off with further discussion and examples of the ultimateproximate distinction. Additionally, this chapter discusses how evolutionary theory may especially be well-suited to inform researchers about the a priori feasibility of proximate hypotheses, and how it can serve as a meta-theory for HBT. Reviewing empirical literature on risk-taking, we examine how likely it is that evolution equipped human psychology with a motivation - or 'drive' or 'instinct'- to be healthy. The chapter further reviews how evolution can help make sense of variation in (and clusters of) unhealthy behaviors resulting from circumstances in early child development. Literature is reviewed showing that such phenomena are likely the result of adaptive calibration to early environments - as opposed to maladaptive developmental outcomes. The reviewed evolutionary literature in this chapter, therefore, predicts that targeting functionally relevant motivations (potentially through environmental intervention) may effectively engender behavior change.

Chapter 3 describes the results of two cross-sectional empirical studies. The studies examine how trait pathogen-avoidance motivation part of what has been referred to as the behavioral immune system - relate to health-protective behaviors. The studies used self-reported measures of attitude and intention to test for STI, and history of casual sex encounters, as indicators of health-protective behavior. It was predicted that individual differences in pathogen-avoidance motivation, assessed using pathogen disgust and germ aversion measures, would positively relate to people's inclination to screen for infectious disease. Further, it was expected that motivations to avoid pathogens would also predict people's tendency to engage in casual sex - as a secondary health-protective effect of pathogenavoidance mechanisms.

An additional implication of a dedicated motivational for pathogenavoidance is the expectation that such motivations may be state- and context dependent. The idea that environments are critical to understand the arousal of specific goal-states is central to the broader evolutionary motivational frameworks as well (e.g., Aunger \& Curtis, 2013; Mark Schaller et al., 2017). Cues to pathogens (activating a pathogen-avoidance motivational state) have, for example, been shown to relate to safe-sex motivations and practices (Meertens et al., 2013; Tybur et al., 2011).

Chapter 4 reports work that addresses how state-activation of pathogen-avoidance relates to health-protective motivation. We hypothesized 
that temporarily activating pathogen-avoidance motivation, through eliciting pathogen-disgust, would influence participants' attitude towards STI testing. Given that attitudes are seen to arise from specific behavioral beliefs (the consequences of a behavior), it was also examined whether beliefs relating to pathogen-avoidance would more strongly predict attitudes - as compared to motivationally irrelevant beliefs.

Chapter 5 contrasts with the other chapters, as opposed to testing health-promoting motivations, here is it was sought whether similar pathogen-avoidance motivations may underlie negativity towards people who get tested for STI. It was hypothesized and contrasted the relevance of two such potential threats: avoidance of infectious and promiscuous individuals. By crafting vignettes describing various different individuals, this chapter explores whether merely getting tested for STI already increases negativity towards those getting tested, and - if so - which motivation relates to negative attitudes and attributed shame most clearly.

Chapter 6, lastly, provides an overview of the main findings in this dissertation. The theoretical and practical implications of the work are subsequently reviewed, as are the studies' limitations and directions for future research. The chapter also discusses the question 'what evolutionary perspectives add to health psychology?', and ends with a conclusion. 

Chapter 2: Function and fitness: The added Value OF AN EVOLUTIONARY PSYCHOLOGICAL PERSPECTIVE IN EXPLAINING HEALTH BEHAVIOR

This chapter is based on: Gruijters, S.L.K., Tybur, J.M., Massar. K., \& Ruiter, R.A.C. (2018). Function and fitness: the added value of an evolutionary psychological perspective in explaining health behavior. Manuscript in preparation. 


\begin{abstract}
The health psychology literature contains a plethora of theories aimed at increasing the field's understanding of health behavior. Even so, an additional perspective may lead to a deeper understanding of health behavior: evolutionary psychological theory. The central premise of this paper suggests that evolutionary theory offers a unique approach for understanding health-protective and health-risk behaviors by analyzing behavior using a level of analysis distinct from those used by sociocognitive theories. It does so by examining the evolved functions of the psychological mechanisms underlying behavior. Increases in our understanding of health behavior gained via such a functional analysis may lead to the generation and testing of novel hypotheses. The current paper provides an introduction to this evolutionary perspective on health behavior. We further illustrate, review, and discuss the relevance of considering evolutionary perspectives in health psychology research.
\end{abstract}

Keywords: Evolutionary psychology, ultimate-proximate explanations, pathogen-avoidance, life history theory, risk taking, early-life conditions 
An intelligent couple can read their Darwin and know that the ultimate reason for their sexual urges is procreation. They know that the woman cannot conceive because she is on the pill. Yet they find that their sexual desire is in no way diminished by the knowledge. Sexual desire is sexual desire and its force, in an individual's psychology, is independent of the ultimate Darwinian pressure that drove it. It is a strong urge which exists independently of its ultimate rationale (Dawkins, 2009, p. 253).

\section{Introduction}

In a recent paper, Burke (2014) asks why researchers in psychology have not - en masse - adopted evolutionary theory to guide their research. This question is especially pertinent for health psychology, a field that has scarcely considered evolutionary theory as a meta-theory of health behavior in its research (Tybur et al., 2012). But, even if evolutionary theory is supported by an impressive array of empirical findings across disciplines (Dawkins, 2009), does it have anything to offer health psychology? The current paper aims to review this question by examining whether - and if so, how - health psychology can benefit from utilizing evolutionary approaches.

Current health psychology theory recognizes the importance of multiple perspectives on behavior, such as the biological, psychological and social levels included in the biopsychosocial model, for instance (Suls \& Rothman, 2004). Multiple and novel perspectives can lead to a deeper understanding of behavior, and we propose that evolutionary theory can supply a new layer to health behavior theory. Throughout this paper, we suggest that evolutionary theory can inform research in health psychology by both answering existing questions concerning the mechanisms underlying behavior and posing novel questions. More specifically, we argue that considering two important concepts used by evolutionary researchers function and fitness - allows health psychologists to gain deeper insight into health behavior. Optimally, such considerations can aid in shaping novel interventions through more precise predictions about the psychological mechanisms underlying health behavior.

The sections below are organized as follows: First, we discuss the core premises of an evolutionary approach to behavior, and how this perspective could contribute to research in health psychology. Second, we elaborate on some general lessons from modern evolution theory about risk taking and health behavior. Third, we describe how an evolutionary perspective has increased our understanding of individual differences and clustering in health behaviors. Finally, we end by considering applications and implications of this framework for research in health psychology and intervention design.

\section{What does it mean to take an evolutionary perspective?}

Evolutionary approaches have provided a perspective on human behavior complementary to that provided by standard social science approaches. Indeed, Mayr (1961), and later Tinbergen (1963), described this complementarity by noting a distinction between proximate explanations, which were examined by biologists interested in physiology, versus ultimate explanations sought by evolutionary biologists (see also Bateson \& Laland, 
2013; Laland, Sterelny, Odling-Smee, Hoppitt, \& Uller, 2011). Evolutionary psychologists, too, make this explanatory distinction in their understanding of human behaviors, as do researchers in related fields such as behavioral ecology and comparative psychology. Simply put, the ultimate-proximate distinction acknowledges that explanations for behavior can be given in terms of both the (ultimate) evolutionary function of a behavior, and in terms of the (proximate) mechanisms (physiological or psychological) that produce the behavior (e.g., Alessi, 1992; Haig, 2013; Scott-Phillips, Dickins, \& West, 2011).

To illustrate the ultimate-proximate distinction using animal behavior, consider the example of Couch's spadefoot toad - a toad native to the Sonoran desert in the southwest U.S and Mexico (R. H. Webb \& Wilshire, 1983). During fall and winter, this species resides underground, only to emerge to surface in summer after the first heavy thunder storms have taken place. The manner in which the toad's nervous system processes the sound waves produced by thunder and lightning strikes proximately explains the toads' emergence. However, the behavior can also be examined via a functional lens - that is, by testing hypotheses why the toad would respond in this way to the thunder. Researchers have observed that the spadefoot requires shallow puddles or pools to fertilize eggs. Because thunder has usually been accompanied by rainfall over the toad's evolutionary history, the toad has evolved the proximate mechanisms described above. Hence, the correlation between the thunder storms and the formation of puddles and pools (needed for egg fertilization) provides an ultimate explanation - and helps understand the why of the behavior. In Mayr's ultimate perspective, the reason why thunder storms cause behavior change in the toad can be understood by specific reproductive fitness benefits of this causal mechanism - here the formation of shallow puddles required for reproduction. Ultimate explanations - as opposed to proximate ones - explain the function of behavior by considering the effects behaviors have (or have had in the past) on reproductive fitness, a term that can be defined as the number of gene copies successfully passed on to subsequent generations (see eg., Ridley, 2004)1,2. Such an analysis can also be applied to human behavior. Health behavior, specifically, can also be illuminated using both ultimate and proximate levels of explanation.

Consider the increase in risk taking that occurs at the onset of puberty (see e.g. Casey, Jones, \& Hare, 2008), including health, physical and financial risk taking. Findings suggest that these increases are mediated by structural and functional changes in the brain, such as changes in the dopaminergic systems mediating reward and sensation seeking - especially in the presence of peers (Casey, Jones, \& Hare, 2008; Steinberg, 2008). Adolescents might take more risks, relative to adults and children, by overestimating the perceived benefits of specific outcomes (Parsons, Siegel, \& Cousins, 1997), discount the importance of future effects (Green, Fry, \& Myerson, 1994; E. M. Hill, Jenkins, \& Farmer, 2008), and being more 
impulsive (Romer, 2010). These accounts are proximate explanations of adolescent behaviors, answering the 'how' and 'what' questions. Even so, identifying proximate causes of behavior does not address the deeper why explanation for adolescents' discounting of the future, impulsivity, and sensation seeking. Such ultimate accounts of risky behavior can provide additional understanding of the behavior, in turn potentially opening up new avenues for research and intervention practice (see Ellis et al., 2012). But what are potential ultimate accounts of risk taking?

A central premise of evolutionary approaches is that understanding how behaviors affect reproductive fitness may contribute to understanding why and when particular behaviors are performed. For evolutionary scientists, these "why" questions are always answered by considering function and fitness rather than internally generated sensations and cognition, such as anxiety, self-esteem, beliefs, or desires. Functional explanations for increased risk taking after puberty onset have been proposed and empirically tested by evolutionary scientists. One functional account of risk taking hypothesizes a role in female mate choice - risk taking may serve a cue to the mate quality of a potential (sexual) partner. A proposed hypothesis is that risk taking enables displays of fortitude - that is, a signal of having 'more to offer' potential partners, which may bolster the risk-taker's attractiveness (Kelly \& Dunbar, 2001). Empirically testing this functional hypothesis, researchers reported that both social risk taking (e.g., defending an unpopular issue that he/she believes in at a social occasion) and physical risk taking (e.g., trying out bungee jumping) were rated as attractive by participants - although not all risky activities were seen as attractive (Wilke, Hutchinson, Todd, \& Kruger, 2006).

Thus, whereas mechanisms such as sensation-seeking and reward processing provide proximate explanations, risk taking may in some domains functionally serve as a mating cue (e.g. physical prowess and status) bolstering attractiveness - an ultimate account of the behavior. This ultimate perspective then also elucidates why increases in risk taking, through alterations in previously described reward-seeking mechanisms, coincide with reproductive maturity (see Casey et al., 2008; Steinberg, 2008) - the period is key a inflection point in mating potential and interest (see also Ellis et al., 2012).

\section{Can ultimate perspectives help health psychologists?}

We can make inroads into understanding "why" questions about behavior by proposing and testing functional hypotheses, as illustrated with the risk taking example. As we will further argue, understanding functions of behavior could lead to novel predictions and more accurate descriptions of the psychological mechanisms underlying health behaviors. Additionally, evolutionary theory might especially be well-suited to inform researchers about the a priori feasibility of proximate hypotheses for psychological 
phenomena (Burke, 2014) and can serve as a meta-theory capable of gauging the plausibility of hypotheses in health psychology.

To give an example of how evolutionary perspectives can inform the plausibility of working hypotheses and further generate new hypotheses, consider work on perceptions of race (Cosmides, Tooby, \& Kurzban, 2003; Kurzban, Tooby, \& Cosmides, 2001). Decades of research suggested that race, like sex and age, is a feature of individuals that is encoded automatically. These findings have been interpreted as suggesting that race is a primary perceptual dimension on which individuals are automatically categorized (Taylor, Fiske, Etcoff, \& Ruderman, 1978). The proposal of a perceptually engrained 'race' category carries the corollary that such a system has been shaped by natural selection- that is, that being able to distinguish individuals based on 'race' served a specific function in human's past.

Taking an evolutionary perspective raises an additional question - why would humans automatically encode race as a feature of a person? Whereas our ancestors regularly encountered individuals of different sexes and ages and encoding an individual based on these features might give some reliable information regarding their likely behaviors, threats, and affordances humans did not evolve in environments in which they interacted with members of different "races" (Cosmides et al., 2003). Kurzban et al. (2001) hypothesized that the race encoding effect might instead be a byproduct of some other functional mechanism, specifically, one that detects cues to alliances and coalitions within smaller social groups. Based on this hypothesis, these authors gathered data that increased our understanding of the proximate mechanisms underlying race categorization. Findings suggested that, when coalition encoding was fostered by shared appearance cues (such as similar clothing, e.g.), race encoding- measured in memory confusion protocol - dramatically decreased (see replications by Pietraszewski, Cosmides, \& Tooby, 2014; Voorspoels, Bartlema, \& Vanpaemel, 2014). Can similar evolutionary insights shed new light on research in health psychology?

One important insight comes from considering health-damaging behaviors (e.g., smoking, unsafe sex, fast driving, drug use etc.) from an evolutionary perspective. At first glance, these behaviors do not seem to have fitness-enhancing functions. In this sense, one can ponder why evolution did not shape an overall 'health motivation' as part of our evolved psychology - steering individuals away from health damaging behaviors. After all, shouldn't natural selection have favored a health promoting psychology, since good health leads to increased survival chances? In fact, poor health is especially well understood within an evolutionary framework. Central to most of these ideas is the observation that an individual's health only marginally correlates with an individual's reproductive fitness. That is, health (and even survival) are only fitness promoting in as much they increase organisms' reproductive fitness (e.g., Buss, 1997). Parental altruism 
(or sacrificial behavior) towards offspring is one clear instance where costly behaviors are nonetheless beneficial to parents' reproductive fitness (see Kurzban et al., 2015). Notably, the fact that self-preservation and reproductive success are in certain contexts even negatively correlated has the potential to elucidate many risky health behaviors (see Tybur et al., 2012).

One aspect of Darwin's original formulation of his theory of evolution already accounted for some of these risk taking phenomena through the process of sexual selection. Darwin (1872) described the diverse and conspicuous secondary sexual characteristics of birds, many of which initially appeared detrimental to the animals' survival probability, since it aids survival to be inconspicuous. There are various ornaments that Darwin noted the birds use to attract mates, including strong musk odors released during the mating season to attract mates, plumes and lengthened feathers 'springing from all parts of the body' (p.232) - often illustrated by the Peacock's aesthetically pleasing tail. Darwin theorized that the reproductive benefits of these ornaments must have outweighed the negative consequences to the individual's immediate health, well-being, and even survival (e.g., alerting predators to the birds' locations). In other words, the reproductive benefits these traits have are 'worth the risk'. Authors have hypothesized that behaviors related to the process of sexual selection may illuminate risk behaviors in our species as well. The central hypothesis is that various risky behaviors (especially in adolescence and young adults) may be functional and behavioral analogues of the peacock's tail.

For example, one study used sexual selection theory to better understand so-called 'drinking games' (Hone, Carter, \& McCullough, 2013); a phenomenon in which young adults drink excessive (and health detrimental) amounts of alcohol in a limited time-frame. The functional hypothesis tested in Hone et al.'s (2013) study was that participation in drinking games may provide a venue for male intrasexual competition, and an opportunity to display physical prowess, fortitude, and willingness to take risks, and at the same time, it may be driven by female intersexual choice (see also Hone \& McCullough, 2015). In line with this hypothesis, mating effort - operationalized as participants' number of past casual sex encounters and their preference to engage in uncommitted sex - predicted alcohol game participation frequency and the amount of alcohol consumed during a drinking game. Thus, these findings give some support for the hypothesis that such risky activities involve mating motivations.

Further evidence for the functional hypothesis that risk taking serves to display physical prowess was found in a field study on skateboarders (Ronay \& Hippel, 2010). In this study, researchers asked skateboarders to perform a number of tricks in front of either a male experimenter or an attractive young female experimenter. Consistent with hypotheses, risk taking (e.g., the number of crash landings) was higher in the female-experimenter condition. The researchers also suggested that increases in testosterone 
might be a proximate mechanism underlying this effect. Confirming this hypothesis, post-experiment testosterone levels were higher for skateboarders in the female experimenter condition.

As another example, researchers investigated how priming female participants with a mating motive would influence risky health behavior (as measured by the frequency to go tanning and to take dangerous diet pills) (S. E. Hill \& Durante, 2011). Women primed with mating related motives, using attractive men and women as prime (respectively triggering intersexual and intrasexual competition), increased their willingness to go tanning or take diet pills. The authors suggested that such results can be explained from a health-belief perspective, if the activation of such a motive influences the actual health beliefs themselves. Indeed, their results showed that women in the experimental condition, where mating motives were primed, indicated feeling less susceptible to the negative consequences of tanning and dieting pills.

A similar paradigm was used to examine the effect of viewing photos of attractive women on males' self-control over smoking (Chiou, Wu, \& Cheng, 2015). Participants were males that had previous intentions to quit smoking. Those exposed to photos of attractive women were more likely to discount future health concerns, and instead gave in to their impulse to smoke, indicating a loss of self-control.

These studies illustrate that motivations with more immediate (and, perhaps, more certain) fitness consequences can be predicted to be prioritized over knowledge about potential long-term health damaging effects. The behavior examined by Hill and Durante (2011) involves, on the ultimate level, a fitness trade-off between the benefits of achieving a mating goal by increasing attractiveness and thereby increasing the odds of acquiring a romantic partner, and self-protective behavior- avoiding risky health behaviors. Similarly in the study by Ciou, Wu and Cheng (2015), where health concerns were discounted due to the priming of a mating mindset. A trade-off seems to be made between motivations towards mating goals, and willingness to incur health costs to this end. Thus, because healthprotecting behaviors are not always the types of behaviors that our evolved psychologies process as increasing fitness, the trade-offs in fitness associated with such behavior provide important and better insight into what motivates risky health behavior.

\section{Evolutionary psychology provides a meta-theory of health behavior theories}

As argued above, evolutionary perspectives challenge the intuition that humans have any adaptations for general 'self-preservation,' or, indeed, general health promotion ${ }^{3}$ (Tybur et al., 2012). Such a perspective would amount to assuming that behavior is consistently guided by health outcome expectations - or risk perceptions - so that every behavior for which a negative health outcome is anticipated is avoided. This insight underlines an 
additional strength of applying an evolutionary perspective in the field of health psychology: it informs the plausibility of other theoretical models. Indeed, this is why one might refer to evolutionary psychology as a "metatheory" (cf. Ronay \& Tybur, 2017) - as an overarching theory of motivation and cognition, it can organize the localized sub-theories often used in health psychology. If a health behavior theory conflicts with evolutionary theory, this raises doubts about the validity of the sub-theory.

To illustrate this meta-theoretical application of evolutionary psychology, consider the terror management approach to health motivation (Arndt \& Goldenberg, 2017; Goldenberg \& Arndt, 2008). This model proposes that many health behaviors are motivated by existential anxieties, an assumption that is critically tied to a consideration of evolution theory. Specifically, the model maintains that the fear of death itself ultimately serves the instinct for self-preservation, [...] a very basic and primitive psychological adaptation that functions to perpetuate the organism's genes' (Pyszczynski, Greenberg, \& Solomon, 1997, p. 5). According to Arndt and Goldenberg (2017) the terror management health model 'offers a foundation for understanding how people manage existential insecurity as well as harnessing the effects of death-related thought to engage productive health behavior change' (Arndt \& Goldenberg, 2017, p. 130).

Despite Terror Management Theory putatively being grounded in evolutionary theory (e.g., Landau, Solomon, Pyszczynski, \& Greenberg, 2007; Pyszczynski, Greenberg, \& Solomon, 1997), its core theoretical assumptions conflict with broader evolutionary psychology. Indeed, as Kirkpatrick and Navarrete (2006) put it, 'in several crucial ways [terror management theory] is entirely out of step with contemporary understandings of evolution by natural selection' ( $p$. 288). For example, one key premise of terror management theory is that 'humans share with other forms of life a basic instinct for self-preservation' (Pyszczynski et al., 1997), which serves as an initial premise for further reasoning and hypotheses about the role of the fear of death as a motivator of health behavior in the terror management health model. As the previous sections have illustrated, the existence of a 'survival instinct' - and general health motivation - is dubious. The various risk taking behaviors, for example drinking games and mate competition, seen through the functional lens of sexual selection provide clear counterexamples of behaviors that promote poor health, but are nevertheless beneficial to fitness (see Buss, 1997).

Additionally, although some of our evolved motivations might appear to encourage self-preserving behavior, they need not be motivated by a domain-general self-preservation motive. Such goals are likely to be attained indirectly, through more functionally specialized, domain-specific motivations. For example, avoiding infectious disease requires a suite of perceptual mechanisms (e.g., detecting the smells and sights indicative of pathogens), emotional motivations (e.g., the emotion disgust), action patterns (e.g., facial expressions that seal the eyes, mouth, and nose from 
pathogens), and behaviors (e.g., withdrawl, hand washing) distinct from those required for avoiding being mauled by a tiger. Other behaviors with health implications (e.g., alcohol consumption versus wearing a seatbelt or avoiding sexually transmitted infections) are further undergirded by distinct motivational systems with distinct functions. This perspective can illuminate many health-compromising behaviors.

\section{Health motivation frameworks from an evolutionary perspective}

If humans have not evolved a general health motivation, then which, if any, parts of our psychology motivate us to engage in health promoting rather than health compromising behaviors? More than being interested in the historical selective pressures that have shaped human psychology, evolutionary psychologists focus on the current results of historical evolutionary processes (e.g., Cosmides \& Tooby, 2013). The historical events that led to so such functional mechanisms are themselves not directly tested by evolutionary psychologists, just as they are not tested by most researchers who study animal behavior from an evolutionary perspective. Instead, thinking in terms of fitness effects serves as a starting point for unravelling a function of a trait in terms of the recurrent ecological or social problems they were suitable to solve for our ancestors (Cosmides \& Tooby, 2013). Evolutionary psychologists often work from a notion that such problems have resulted in the evolution of a collation of a large number of domain-specific computational mechanisms.

Multiple evolutionarily-informed frameworks of motivation have arisen in the literature (e.g., Aunger \& Curtis, 2013; Bernard, Mills, Swenson, \& Walsh, 2005; Kenrick, Neuberg, Griskevicius, Becker, \& Schaller, 2010b). Although these approaches differ in their specific postulates, they share a similar approach to understanding the motivational systems involved in behavior. Each begins with a consideration of problems that posed recurrent challenges to inclusive fitness during human's evolutionary history - the types of considerations that allow us to generate hypotheses of the factors that influence contemporary human behavior, including health behavior. Such problems include those related to acquiring a mate, parenting, affiliating, avoiding infectious disease, and protecting the self from violent attacks.

Recent work on pathogen-avoidance motivation (e.g., Curtis, 2011; Curtis, de Barra, \& Aunger, 2011; Tybur, Lieberman, \& Griskevicius, 2009; Tybur, Lieberman, Kurzban, \& DeScioli, 2013) can serve as an example of a domain-specific problem that led to adaptations regulating health-protective behavior. Pathogens (i.e. viruses, bacteria, parasites, fungi etc.) have posed fitness challenges to humans, and they continue to do so. These challenges include draining of metabolic resources, producing of visual effects that lead to social exclusion, reducing of attractiveness to mates, and being directly or indirectly deadly. Researchers have argued that pathogens have not only shaped the evolution of the innate and adaptive immune system, but also a 
set of psychological defenses. This additional set of defenses dedicated to neutralize the risk of pathogens has been referred to as a 'behavioral immune system' (Mark Schaller \& Duncan, 2007; Mark Schaller \& Park, 2011), an important motivational component of which is the emotion disgust (Curtis, 2011; Curtis, Aunger, \& Rabie, 2004; Curtis et al., 2011; Oaten et al., 2009; Mark Schaller, 2014; Tybur et al., 2013).

Tybur, Bryan, Magnan, \& Hooper (2011) devised an experiment in which they manipulated a pathogen-avoidance motive using an olfactory cue to pathogens. Before participants entered the lab, the experimenter sprayed a single-dose of a spray resembling the smell of feces, which was not explicitly noticeable by the participants. Compared to a non-sprayed control group, these researchers found that this implicit cue to pathogens (i.e. a foul smell) caused participants to indicate an increased willingness to use condoms in future sexual encounters, buy and carry condoms with them, and discuss condom-use with future sex partners. Similar domain-specific input regulating health protective behavior were observed in a study where $\mathrm{t}$ participants were exposed to vignettes describing events after a night out in town (Meertens, Branković, Ruiter, Lohstroh, and Schaalma, 2013). Participants were asked to imagine that they went home with someone after a night out, had unsafe sex, and that the next morning they either woke up in a tidy room (control condition) or a very sloppy, even dirty room. Next, they were asked to rate the likelihood of having contracted a sexuallytransmitted infection, and to indicate the probability of engaging in this behavior again. Results supported the researchers' hypotheses, and indicated that participants in the dirty environment condition felt more susceptible to having contracted a sexually-transmitted disease, and also were less inclined to repeat such behavior in the future.

Note that the results of both studies involve implicit responses to cues to pathogens, influencing health decisions in these contexts. Why would such diverse cues (a dirty room or a bad odor) have such influence on health-protective behavior? From an ultimate perspective, such findings can be elucidated by considering the fitness effects in the responses to pathogen cues: they steer individuals away from pathogens. The changes in risk perceptions and attitudes by such cues can thus be seen to reflect the ultimate motive behind the behavior: avoiding infectious disease. The assumption here is that a narrow band of ecological conditions has reliably correlated with actual pathogen presence, and humans evolved to perceive these conditions as aversive (and, indeed, disgusting). Indeed, typical known causes for infection (dirt, gore, rotten foods, blood, feces) are listed as common causes of infection in medical textbooks - supporting the idea that disgust thus functions to prevent disease contraction (Curtis, 2013). Note that this 'disease-avoidance' system is bound to pathogen risk; the domainspecificity is salient when we consider the sort of inputs that trigger avoidance-behavior. Specific perceptual cues, such as foul odors, bad taste, or visual abnormalities (or the elicited disgust), have been argued to be 
important proximate causes of domain specific pathogen-avoidance (Tybur et al., 2013).

Importantly, conceptualizing the motivational system behind pathogenavoidance as a domain-specific psychological adaptation does not necessarily imply that behavior motivated by pathogen-avoidance only (or always) results from the actual presence of pathogens (Tybur \& Lieberman, 2016). Since pathogens cannot be detected directly, the cognitive systems regulating pathogen-avoidance are faced with a signal detection problem, which leads to false-alarms. This situation is similar to health care providers who test individuals for HIV; any such test involves a probability of false positives and false negatives (depending on sensitivity and specificity). Such cognitive signal-detection problems have been extensively examined in the literature (Haselton et al., 2000; Neuberg et al., 2011), and are of importance for understanding health behavior as well.

For example, Gruijters, Tybur, Ruiter, and Massar (2016), tested how measures of trait pathogen-avoidance motivation and related to attitudes and intentions towards various health-protecting behavior. These healthprotective attitudes and intentions were clustered in two groups. One set of outcomes (e.g., STI testing after unsafe sexual intercourse) related to pathogen-contagion risk, the other cluster of variables (e.g., doctor visit for frequent headaches) did not relate to pathogen-contagion risk. The results revealed that both types of health-protective behaviors were predicted by trait pathogen-avoidance motivation. Proximately, the observation that trait pathogen-avoidance correlated negatively with attitudes and intentions towards both pathogen-related and unrelated health concerns can be explained by an oversensitive detection system which tends to err towards false-positives when faced with ostensible cues of disease, whether it is unsafe sex or frequent headaches. Thus, even non-obvious signs (e.g., noninfectious rashes) of pathogen contraction might cause avoidance by people with high levels of trait pathogen-avoidance; reflecting a biased cognitive solution to a signal-detection problem when faced with ostensible cues to disease.

\section{Making sense of individual differences in health behavior}

Differences in health behavior may be tied to differences in cognition, emotion, and motivation, which can often be understood as the result of functional, fitness promoting mechanisms (e.g. disgust mechanisms and pathogen-avoidance functions). The discussion thus far leaves open an important question - if psychological mechanisms are adaptively shaped by natural selection to fulfill adaptive functions, why do we observe so much variance in health behaviors between individuals? Evolution theory enables us to understand why certain psychological traits converge to particular population mean values (e.g. on average adaptive levels of disgust sensitivity) - this is because natural selection can be expected to shape traits in populations around 'average' adaptive levels. It is also clear, 
however, that most psychological traits show considerable variance. Does this imply that being 'average' on disgust sensitivity means to be better adapted than people scoring very low or high on this continuum?

Recent developments in evolutionary approaches to individual differences suggest that such individual differences may not merely be noise. Instead, some individual differences may reflect different, but equally adaptive, 'behavioral strategies'. Theorists have proposed models that put natural selection central in not only selecting for adaptive traits, but also in explaining heritable variation in traits and shaping capacities that allow plasticity and learning in response to local environments (Nettle, Gibson, Lawson, \& Sear, 2013). The branch of evolutionary theory that captures some of these principles is known as life-history theory (LHT; Buss, 2009; Gruijters \& Fleuren, 2018; Kaplan \& Gangestad, 2005; Stearns, 1992, 1989; Tybur et al., 2012). We first briefly review the model as it used in biology before proceeding to its applications to human behavior.

Central in LHT is the distinction between extrinsic and intrinsic causes of death. Extrinsic causes of death pertain to those uncontrollable factors in the environment (e.g. homicide, accidents), whereas intrinsic causes refer to those causes of death that are under the control of physiology (e.g. repair mechanisms for cell death). One tenet of LHT proposes that, when extrinsic causes of death are high, selection can be expected to 'speed up' the reproductive cycle of a species. Species characterized by such so-called 'fast' life history strategies tend to mature earlier, reproduce more quickly, and allocate less energy to maintenance of the body (i.e. less attempts to reduce intrinsic mortality causes). Those exposed to fewer extrinsic causes of mortality may have protracted development and maturation, reproduce later, and have longer life-spans (so called 'slow' life history strategies). In evolutionary biology, the theory has been applied to explain why some species grow old yet produce fewer offspring (e.g., elephants) than other species which reach senescence at young age yet produce many offspring (e.g., mice).

Important in LHT is the notion that extrinsic mortality puts limits on how much energy organisms invest in their development and maintenance of health. Nettle (2010a) offers a useful metaphor: 'one would not spend too much on repairing a car in an environment where cars are frequently stolen anyway' (p.2). Evolutionary psychologists interested in human behavior extended LHT to also explain 1) heritable individual differences in human psychology, but also 2) the effects of early exposure to harsh and stressful environments on development. Here, we focus on the latter implication of LHT which may carry more direct implications for health psychology research. 


\section{Early-life experiences, socioeconomic status, and variation in health behaviors}

Evolutionary-developmental models have begun to capture how earlylife conditions can reverberate from childhood into psychological adulthood (Belsky, Schlomer, \& Ellis, 2012; Frankenhuis \& de Weerth, 2013; Nettle, Frankenhuis, \& Rickard, 2013). A central prediction of LHT is that when environments provide little security or stability, i.e. when infants experience stress in the first few years of life, development can be "pushed" towards faster paced LHT strategies. Variables typically interpreted as reflecting fast life history strategies include increased risk taking, early reproduction, a higher number of offspring, and increased casual sexual encounters (Figueredo et al., 2005; Simpson, Griskevicius, Kuo, Sung, \& Collins, 2012).

LHT also offers novel interpretations of the causes of differences in health behaviors observed between individuals with a different socioeconomic status (SES). Lower SES is associated with a variety of risky and health damaging behaviors, including poorer diet and less physical exercise, more smoking (e.g., Brennan, Henry, Nicholson, Kotowicz, \& Pasco, 2009), more alcohol consumption and drug use (e.g., Méjean et al., 2013), less compliance with health advice, more violence and various other health damaging behaviors (see Nettle, 2009; Pepper \& Nettle, 2014, 2017, Stringhini et al., 2010, 2017). Empirical research has shown that individuals who grew up in low SES environments tend to eat more in the absence of energy needs (S. E. Hill, Prokosch, DelPriore, Griskevicius, \& Kramer, 2016), which may link to the higher obesity rates amongst low SES individuals (see Ahlstrom, Dinh, Haselton, \& Tomiyama, 2017).

Many proximate explanatory variables have been proposed to explain SES differences in health behaviors, including a sense of lack of control over health outcomes and a decreased interest in long-term consequences of current behaviors (Nettle, 2010b). However, only recently have researchers aimed to account for the ultimate causes of such systematic variance in health behavior. An important insight here is that people grow up in very different environments; in some of these environments (e.g. low SES neighborhoods) extrinsic mortality causes are much more salient (and likely) compared to higher SES environments (see Stringhini et al., 2017). Authors have argued that SES-related differences in health behavior may in fact be adaptive responses to harsh developmental environments, instead of merely maladaptive consequences of deprived social environments (Pepper \& Nettle, 2017).

LHT predicts that it would be beneficial in unstable environments to have mechanisms operating in development allowing fine-tuning of life history strategy- a developmental effect that Pepper and Nettle (2017) label a contextually appropriate response. Early-life conditions, thus, are expected to influence how people make present-future tradeoffs in adulthood. The increased focus on the present that has been tied to SES (Nettle, 2009; Pepper \& Nettle, 2014, 2017, then, may ultimately reflect an adaptive 
response to deprived developmental environments. This contextually appropriate response to deprivation is assumed to be proximately reflected in a short-term time preference - including a focus on immediate rewards and discounting of future rewards (e.g., Griskevicius, Tybur, Delton, \& Robertson, 2011), higher levels of impulsivity (e.g., Frankenhuis, Panchanathan, \& Nettle, 2016), and pessimism about the future (Robb, Simon, \& Wardle, 2009).

Further evidence for early-life influence on the calibration of life history strategy can be gleaned from studies on childbirth timing. Research found that in deprived relative to affluent communities the age of first childbirth is younger (Nettle, 2010a ). In an experimental set-up, it was also found that manipulating mortality cues increased the desire to have children soon rather than later. This effect was however only observed in individuals with a lower SES background (Griskevicius, Delton, Robertson, \& Tybur, 2011).

LHT predictions have been tested to understand variation in health behavior in the general population as well. Individuals exposed to unpredictable conditions during development are more likely to discount the future effects of behavior, predicting in turn risk taking behavior (E. M. Hill et al., 2008), such as risky drinking (E. M. Hill \& Chow, 2002). Abused children are more sensitive to detect and recall information related to potential threats (Frankenhuis \& de Weerth, 2013). Exposure to harsh environments while growing up has also been linked to higher levels of anxiety in later life (Nettle, 2009b). Simpson et al (2012) observed that individuals who had been exposed to unpredictable, changeable environments during infancy exhibit inceased levels of risk taking behavior across several domains - including sexual risk taking, aggression, and criminal behavior - in their twenties. Another proposed variable that may relate to faster life history strategies is subjective life expectancy (i.e. how old someone expects to become). Wang, Kruger and Wilke (2009) found that people's estimates of their life expectancy predicted risk taking across several domains, including health related risk taking (e.g., having unprotected sexual intercourse) - suggesting that this variable also captures in life history strategy. These models thus also suggest that a lot of variance in health behaviors may be explained by variation in life history strategy, and calibration of the strategy in response to early-life conditions (see also, Ellis, Figueredo, Brumbach, \& Schlomer, 2009; Nettle, 2010a, 2009, 2010b; Pepper \& Nettle, 2014).

In sum, LHT merges evolutionary and developmental approaches by viewing developmental plasticity in response to local environments as an evolved adaptation in itself; selected because of the advantageous ability to respond flexibly to environmental circumstances (Nettle \& Bateson, 2015). This is a novel layer to traditional evolutionary thinking which - in a way fuses the evolutionary and developmental approaches to psychology. Instead of assuming that individuals are born with certain dispositional levels of a trait, LHT emphasizes that early life-experiences influence development in 
adaptive ways. Variation in behavior can be explained through adaptive developmental processes that fine-tune individuals' life history strategy. Individuals growing up in impoverished or in unpredictable environments are expected to develop an increased focus on mating which accompanies a decreased focus on health protective behaviors in adulthood - illustrated by the discussion of the relationship between SES and health. LHT not only provides a framework for understanding the ultimate roots of such variation; it also has the ability to elucidate the clustering of health behaviors.

\section{Explaining clustering in health-risk behaviors}

Research has shown that health-risk behaviors (e.g., smoking and alcohol consumption) tend to correlate positively with each other (e.g., Lippke, Nigg, \& Maddock, 2012; McAloney, Graham, Law, \& Platt, 2013; Noble, Paul, Turon, \& Oldmeadow, 2015). Therefore, rather than examining and changing risky health behaviors separately, these suites of correlated behaviors could be considered part of more general behavioral patterns. This carries the implication that teaching individuals to change one behavior, may transfer to change in other behaviors. This suggestion and interpretation bears resemblance to the work of life-history theorists. Factor analyses of the covariance between various health behaviors have found that a constellation of behaviors (e.g. promiscuity, orientation on the present, risk taking behaviors) show strong positive interrelations (Figueredo et al., 2005; Figueredo, Cabeza de Baca, Woodley, de Baca, \& Woodley, 2012; Figueredo, Vasquez, Brumbach, \& Schneider, 2007). LHT may provide a functional account of the observed clustering of health behaviors. LHT explains clustering in terms of a tradeoff resulting from a fast life-history strategy, that is, more investments in direct reproductive goals. In the context of the early-life influence associated with SES, Pepper and Nettle (2017) described such clusters of health behaviors as the 'behavioral constellation of deprivation'.

Overall then, the clustering of health risk behaviors can be viewed as manifestation of a life history strategy - to psychologically implement such a strategy various traits need to be fine-tuned differently within individuals. Extending Nettle's (2010) metaphor, a well-designed race car requires not only the appropriate sort of tires, but also a highly powered engine and limited overall weight. Conversely, the design of car that is not meant for speed but sustainability would require various other choices of characteristics. Just as an eye for design makes predictions regarding correlations among race car features (tires and engines), in a similar manner, the ultimate perspective provided by life history strategy may help understand the correlations among health-risk behaviors.

\section{Discussion}

We have discussed several aspects of evolutionary theory, its premises, and examples of theorizing that can aid our understanding of health behavior. But is such theory of practical importance to health psychologists, 
for example in the design of health interventions? Below we discuss examples of ways in which this knowledge can be transferred to ongoing research in health psychology. Summarizing the previous sections, we discuss how key insights from evolutionary psychology can be used by health psychologists.

Behavior change interventions can be designed to target, and work in synergy with, functional aspects of behavior

Throughout this paper, we have discussed risk taking, a phenomenon which is at the root of many unhealthy behaviors and health outcomes, and therefore of particular importance to understand for health psychologists. Evolutionary psychology's ultimate-proximate distinction allows a functional understanding of risk taking by focusing on the fitness cost-benefits tradeoffs involved in behaviors. One hypothesis about the function of risk taking behavior was drawn from sexual selection theory - different types of risk taking may have benefits in terms of conveying domain-specific (e.g. physical, financial, social) prowess to others, which is assumed to increase mate value (Ronay \& Hippel, 2010; Wang et al., 2009; Wilke et al., 2006). While such behaviors may be deemed functional viewed from an ultimate perspective, we have reviewed literature showing that often such motivations constitute a tradeoff with health costs - such as consequences of tanning and use of diet pills (S. E. Hill \& Durante, 2011), smoking (Chiou et al., 2015), alcohol consumption (Hone et al., 2013; Hone \& McCullough, 2015), or physical risk taking (Ronay \& Hippel, 2010).

Some implications for policy and intervention development of a functional perspective have recently been reviewed with respect to adolescent risk taking interventions (Ellis et al., 2012). In line with our discussion, Ellis et al. argued that to frame adolescent risk taking as maladaptive - dysfunctional - provides an incomplete understanding of why adolescents would engage in health risk behaviors. Instead, some of such risk taking behaviors may be ultimately strategic. Putting the ultimate question central ('what is in it for the kids?', p. 600), these authors offer several insights in risky adolescent behaviors, which have consequences for some tactics to engender behavior change with interventions.

For example, by considering adolescent risk behavior as functional, it may be counterproductive and undesirable to aim to eliminate risk taking in general, if risk taking is an inherent part of some individuals' developmental trajectories. For instance, since adolescence is a transition period in which reproductive and social goals such as status, dominance, and prestige become prioritized (for a discussion, Ellis et al., 2012), attempts to persuade teenagers to simply 'give up' their means of reaching such goals (e.g. through risk taking) may be doomed to fail. Instead, interventionists might aim to reduce the detrimental consequences of risk taking by providing safe and healthy alternative means of attaining these goals. This example illustrates that it is important to understand the goals that people are expected to prioritize, and the available evolutionary psychological 
frameworks in the literature may aid intervention developers to this end (Aunger \& Curtis, 2013, 2016; Kenrick, Griskevicius, et al., 2010; Neuberg et al., 2011; Mark Schaller et al., 2017).

Motivations underlying health behaviors reflect domain-specific motivational systems rather than a domain-general motivation to stay healthy

The assumption that people can reasonably be expected to pursue and invest in health goals, i.e.to expect that all individuals possess a general health motivation, is implicitly and explicitly present across health behavior theories. The Terror Management Health Model (Arndt \& Goldenberg, 2017; Goldenberg \& Arndt, 2008) is one example of a theory making such an assumption explicit; it postulates a general instinct for self-preservation to be a central human motivation. This assumption is also implicit to reasoning in models such as the Health Belief Model (Janz \& Becker, 1984; Stretcher \& Rosenstock, 1997) and Protection Motivation Theory (Floyd et al., 2000; Rogers \& Prentice-Dunn, 1997). Core variables in these models include perceptions of susceptibility and severity of a particular health condition, clustered under threat appraisal in Protection Motivation Theory. Implicit in these models is that any health threat - given high levels of selfefficacy (Peters et al., 2013) - can reasonably be expected to increase protection motivation and health-protective behavior, and thus these models assume a generalized health motivation. In this paper, we have argued against this assumption (see also Tybur et al., 2012). We do not imply that these variables are not important predictors of health behavior; instead we suggest that evolutionary-perspectives on motivation can guide more specific hypotheses on when threats can be expected to meaningfully influence health-protective behaviors. The discussed evolutionary psychological models of motivation instead suggest that behavior is shaped by the interaction of multiple, more tangible, and context and state dependent motivations (Kenrick, Griskevicius, et al., 2010; Kenrick, Neuberg, et al., 2010; Neuberg et al., 2011).

For example, unsafe sexual practices may result from tradeoffs between mating motivations and pathogen-avoidance motivations (see Gruijters et al., 2016). If one does not assume a general health-motivation, there is no clear priority (or hierarchy) of one motivation over the other; instead the weighting of these motives may be contingent on the context (e.g., Meertens et al., 2013) and state-dependent (Tybur et al., 2011) - providing opportunities for the creation of behavior change methods. Threat information may have a limited impact on behavior under circumstances where specific other motivations can be predicted to be prioritized, e.g. when adolescents are in the company of peers. More research on when and where such motivations may be prioritized can benefit the design of effective interventions.

Variation in (and clusters of) unhealthy behaviors can result from functional calibrations to (early) environments.

Much contemporary evolutionary work highlights the role of early environmental influence as being critical to understanding to understanding 
later-life health behaviors and risk taking propensities. As discussed previously, the development of life-history strategies (relating to risk taking behavior) in adulthood is contingent on the stability and predictability of developing children's environment. Fast life history strategies are partly forged in response to harsh or unpredictable early life environmental conditions calibrate cognitive development towards a fast strategy (e.g., Belsky et al., 2012; Frankenhuis et al., 2016; Simpson et al., 2012). This notion is of importance to health promotion practice, as it encourages the design of pre-emptive health behavior interventions during early childhood (see also Ahlstrom et al., 2017).

While the flexibility of life history strategy in early development provides opportunities for interventions, it carries the implication that, once these strategies have taken shape, they may require more elaborate methods to change. Thus, 'Band-Aid interventions' (Ellis et al., 2012, p. 609), such as health education, promoting self-esteem or training coping skills are unlikely to completely counteract such - evolutionary and developmentally engrained - behavioral patterns (Ellis et al., 2012). Instead, evolutionarydevelopmental models suggest that those interventions targeting elements of the early-life environment, may have protracted and lasting beneficial effects on adults' health behavior. Additionally, interventions delivered in this stage may create a shift in clusters of later life health behavior patterns - rather than single behaviors - due to the development of overall slower life history strategies.

Finally, health models of early-life influences and SES (Frankenhuis \& de Weerth, 2013; Pepper \& Nettle, 2014, 2017) emphasize the benefits of a renewed focus on time perspective as key to understanding health risk behaviors (see also Robbins \& Bryan, 2004). This variable, grouping related variables such as temporal discounting, impulsivity, reward sensitivity, and future pessimism (e.g., Pepper \& Nettle, 2014, 2017), may be an important proximate variable underlying the implementation of life history strategy. Research has shown indications that it may be possible to motivate individuals to become more future-oriented through environmental intervention. For example, adolescents' time perspective does not uniformly relate to problem behaviors at school (Chen \& Vazsonyi, 2013); instead, it is moderated by contextual factors, including school's future orientation climate - measured as the average of all students' future orientation scores for a given school.

Overall, the described evolutionary-developmental models emphasize the benefits of increased efforts to target ecological factors to create sustainable behavior change, preferably early-life factors, alternatively through changing behavior contexts in ways that promote healthy behaviors by tapping into functionally relevant motivations (e.g., Biran et al., 2014). The reviewed evolutionary approach to health and health behavior (see also Aunger \& Curtis, 2013, 2016), therefore, predicts that environmental interventions may effectively engender behavior change. In this sense, 
creating health promoting behavioral contexts is a viable addition to - or, a way towards - the creation of health promoting mindsets.

\section{Conclusion}

Evolutionary perspectives on human motivation highlight the tenuous nature of assumptions that people have a general health motivation. Evolutionary psychology could be viewed as yet another perspective on health behavior - one that could be piled on with the myriad other health models. This view would overlook the critical contribution that evolutionary psychology can make to health psychology as a meta-theoretical framework. The application of the ultimate-proximate distinctions clarifies that functions of behavior are independent of the mechanisms that generate behavior, but a convergence of both perspectives leads to a more complete picture of health behavior. By testing hypotheses about the evolved function of behavior, researchers may come to a deeper understanding of the processes driving health behavior.

\section{Footnotes}

1 Formally, theories that consider ultimate causes of behavior explain why genetic correlates of behavior (through their influence on neural, physiological and cognitive mechanisms) were selected for in human ancestors; since the genetic correlates constitute the heritable aspect of behavior. Behavioral geneticists have argued that in principle all aspects of human behavior are to some extend heritable - that is, at least some proportion of trait variance is explained by genetic variance (Turkheimer, 2000). Although a successful evolutionary approach at least to some extent relies on this notion, this does not imply that evolutionary psychologists need to study ultimate hypotheses on a genetic level; rather such hypotheses can be tested as well using neuroscientific, cognitive or behavioral methods. In order to do so viably, evolutionary psychologists require assuming some correlation between heritable genetic variation, and neural/cognitive development.

2 Modern evolutionary theory applies an even broader definition of the term reproductive fitness. This definition not only involves the effect of behavior on an individual's reproductive fitness, but also on one's kin carrying a proportion of identical genetic material. The accrued fitness effects of behavior on an individual and kin is termed inclusive fitness in the literature. In this model, fitness is understood in terms of frequencies of specific gene variants passed on to subsequent generations (whether directly or through relatives), such alternative versions of genes that individuals transmit are referred to as alleles in the literature (Hamilton, 1964; Kurzban, Burton-Chellew, \& West, 2016.) 
Chapter 3: Sex, Germs, and health: PathogenAVOIDANCE MOTIVATION AND HEALTH-PROTECTIVE BEHAVIOR

This chapter is based on: Gruijters, S.L.K., Tybur, J.M., Ruiter, R.A.C., \& Massar, K. (2016). Sex, germs, and health: pathogen-avoidance motives and health-protective behaviour. Psychology \& Health, 31(8), 959-975. 


\begin{abstract}
Recent work suggests that the psychology of pathogen avoidance has wide-reaching effects on how people interact with the world. These processes - part of what has been referred to as the behavioral immune system- are, in a way, our "evolved" health psychology. However, scholars have scarcely investigated how the behavioral immune system relates to health-protective behaviors. The current research attempts to fill this gap. Across two crosssectional studies ( $\mathrm{N}=386$ and 470, respectively), we examined the relationship between pathogen-avoidance motives and health-protective behavior. The studies used self-reported measures of attitude and intention as indicators of health-protective behavior. Data collected in Study 1 revealed that pathogen-avoidance motivation related to participants' attitude and intention towards STI screening. High levels of pathogen-avoidance motivation were also related to having had fewer sexual partners, which partially mediated the effect of pathogen-avoidance variables on testing motivation. Study 2 extended these findings by showing moderate associations between pathogen-avoidance motivation and a broad range of health-protective behaviors, including but not limited to pathogen-related health concerns. We argue that understanding and targeting pathogenavoidance psychology can add novel and important understanding of healthprotective behavior.
\end{abstract}

Keywords: Pathogen-avoidance, pathogen disgust, behavioral immune system, health-protective behavior, STI screening, sexual health 


\section{Introduction}

Viruses and bacteria have infected multicellular organisms for millions of years, and their consequences have led to the evolution of complex defense systems, such as the innate and adaptive immune system. Researchers have recently begun to detail the aspects of our psychology that act as additional, first line of defense against pathogens (e.g., Curtis et al., 2011; Tybur et al., 2013) - these psychological defenses have been described as the "behavioral" immune system (Mark Schaller \& Duncan, 2007; Mark Schaller \& Park, 2011).

Despite the strong recent increase in behavioral immune system research (e.g., Mark Schaller, 2011, 2014; Tybur et al., 2014), empirical research into how to connect the behavioral immune system to health promotion questions remains scarce. Here we aim to fill this gap by reporting two studies that test whether behavioral immune system principles can help explain health-protective behavior. Specifically, we investigate how behavioral immune system principles might be used to understand health-protective behavior, particularly those relevant to sexual health.

Several lines of research have focused on better understanding what leads to behaviors that prevent sexually-transmitted infections (STI), including condom-use (e.g., Albarracin, Johnson, Fishbein, \& Muellerleile, 2001; Bryan, Aiken, \& West, 1996) and post-coital prophylactic behaviors, such as screening for STI (e.g., Lorenc et al., 2011; Mevissen, Ruiter, Meertens, Zimbile, \& Schaalma, 2011). Despite progress in our understanding of the determinants of health-protective behaviors, the consequences of infectious disease remain a significant problem.

Much of the research on STI prevention is guided by social cognitive theories, such as the theory of planned behavior (e.g., Ajzen, 1991; Godin \& Kok, 1996) and the health belief model (Janz \& Becker, 1984; Rosenstock, 1974; Strecher, Champion, \& Rosenstock, 1997). These models propose that personal beliefs (e.g., those related to risk assessment and personal abilities) are the primary determinant of health behavior. Implicit in such models is the assumption that people have conscious access to the most important psychological factors influencing health behavior. Although these approaches to health-protective behavior have been useful in predicting behavior and identifying targets for interventions, a number of additional, lesser examined motivational processes might also underlie health behavior. We propose that pathogen-avoidance motivations are among these.

\section{Mechanisms underlying pathogen-avoidance}

Since infectious microbes are too small to be seen directly, the behavioral immune system has been shaped to monitor for and respond to cues that reliably correlate with the presence of pathogens (Mark Schaller \& Park, 2011). A broad range of perceptual cues - including visual, olfactory, gustatory, and tactile cues - might suggest that pathogens are present, and 
hence evoke a pathogen-avoidance motivational state, such as disgust (see Tybur et al., 2013). Some recent work suggests that these motivations either at a trait or state level - can influence health-promoting behaviors. For example, Tybur, Bryan, Magnan, and Hooper (2011) invited participants into a lab that was sprayed with an odor similar to those associated with pathogen sources. The authors hypothesized that this olfactory cue to pathogens would (implicitly) motivate prophylactic behavior - specifically, that it would increase participants' intention to use, buy, and discuss the use of condoms in future sexual encounters. Results were consistent with this hypothesis - participants in the pathogen prime condition reported greater condom use intentions than participants in a control condition.

Complementing these findings, Meertens, Brankovic, Ruiter, Lohstroh, and Schaalma (Meertens et al., 2013) examined the effect of the physical environment on participants' beliefs about susceptibility to STI. They found that participants who imagined waking up in a dirty room after a one night stand judged their susceptibility to STI to be higher than did participants who imagined waking up in a clean environment after a one night stand. Participants imagining the dirty room also considered the odds of engaging in unsafe casual sex in the future significantly lower.

\section{Sexual behavior and trait aversion to pathogens}

Both of the above examples concerned sexual behavior. This is perhaps not surprising, since sexual behavior with another person reliably put a person at risk of pathogen infections. Intercourse is a major source of risk for bacterial infections such as Chlamydia and Gonorrhea, and viral infections such as HIV and herpes. Further, the close physical contact that co-occurs with sexual intercourse puts individuals at risk for infection via pathogens transmitted through nonsexual fluids (e.g. saliva and sweat), simply because of physical proximity and contact with a partner. These risks might underlie some of the main motivations to not engage in sexual behaviors (Patrick, Maggs, Cooper, \& Lee, 2011). Naturally, there are many benefits to sexual behavior too, such as procreation and experiencing pleasure and intimacy - benefits that engender strong motivations to engage in sexual behavior (e.g., C. A. Hill \& Preston, 1996). Indeed, sexual and pathogen-avoidance motives are likely antagonistically related to each other. That is, relevant goal-states, such as pathogen-avoidance and a desire to experience sexual pleasure may act as opposing psychological forces in motivating behavior.

For example, Borg and De Jong (2012) observed a negative relationship between disgust and feelings of sexual arousal. Such results illustrate the interplay between two competing motivational states; when sexual arousal is high, disgust responses related to pathogen threat may decrease. Additional research has focused on trait variability in pathogen-avoidance and its relationship with sexual behavior (e.g., Duncan et al., 2009; Gangestad \& Grebe, 2014; Tybur et al., 2015, 2009). Some of this work has demonstrated 
that individuals who have higher trait level pathogen avoidance are less inclined to engage in sex with multiple partners, which is a key risk factor for acquiring an infectious disease (Joffe et al., 1992). With regard to sexual health, researchers observed a reliable negative association between trait pathogen-avoidance tendencies and motivations to have sex with multiple partners (e.g., Duncan et al., 2009; Murray, Jones, \& Schaller, 2013; Tybur et al., 2015). This suggests that trait variation in pathogen-avoidance might affect the implicit cost-benefit analysis involved in the decision to have casual sex. Specifically, people with an active behavioral immune system might "judge" the costs of casual sex to be higher than people with a less active behavioral immune system, and thus would have less casual sex and perceive a higher need for health-protective behavior in risky situations (e.g., STI testing after unsafe sex). Overall, we assumed that a basic motivation for pathogen-avoidance might influence attitude and intention towards healthprotective behavior, and we tested these relationships in two cross-sectional survey studies.

\section{Study 1}

In our first study, we examined how individual differences in pathogenavoidance motivations relate to testing for STI1. We examined whether individuals scoring higher on pathogen-avoidance motivation not only have had fewer casual sex partners - thus limiting the chances for pathogen transmission - but whether they are also more inclined to be screened for STI after an act of unprotected sexual intercourse. We expected that history of casual sex is likely a direct predictor of health-protective attitude and intention. Moreover, considering earlier work that directly related pathogenavoidance with sexual behavior (Tybur et al., 2015), we expected history of casual sex to mediate the relationship between pathogen-avoidance and health-protective attitude and intention. Inclinations toward testing for STI were operationalized using measures of attitude and intention, as is commonplace in many behavior prediction models (Ajzen, 1985, 1991). Our hypotheses were as follows:

1. Pathogen-avoidance motivation correlates positively with attitude and intention towards being screened for STI after an act of unsafe sex.

2. Pathogen-avoidance motivation correlates with fewer self-reported past casual sexual contacts.

3. The relationship between pathogen-avoidance and intention towards STI screening is mediated by attitude and history of casual sex.

\section{Method}

\section{Participants and selection procedure}

Using a crowdsourcing platform, Amazon Mechanical Turk (for an evaluation, Buhrmester, Kwang, \& Gosling, 2011) participants from the US 
were invited via an advertisement on Amazon.com to participate in the current study. We limited our recruitment to men and women aged 18-27 years who were not in a romantic relationship. We selected this age group since it presents an at-risk population for STI and because of a larger variance in sexual behavior compared to older populations. A total of 752 complete responses to the survey were recorded. Duplicate responses were determined by IP-matching and exact data matches. Only initial responses from users to the survey were maintained in the dataset.

After exclusion of non-single participants $(n=161)$, duplicate respondents $(n=95)$, exclusion of individuals who did not adequately respond to an integrated attentive reading check2 $(n=79)$, and participants that did not meet our age criterion $(n=31)$, our final sample contained a total of $N=386$ participants, including 228 men (Mage= 23.8) and 158 women $($ Mage $=23.6)$.

\section{Materials and Measures}

Sociosexual Orientation Inventory. We measured participants' history of engaging in casual sex using the Revised Sociosexual Orientation inventory (SOI-R; Penke \& Asendorpf, 2008). The SOI-R includes nine items, measured on 9-point Likert scales, assumed to be indicators of three factors: 1) desire to have casual sex, 2) attitude towards having casual sex, and 3) actual history of having casual sex. Since we are mainly interested in sexual behavior, as this is the actual risk factor for disease contraction, we only included the behavior facet of the SOI-R in our analyses.

Pathogen-avoidance. We measured individual differences in pathogenavoidance using the Perceived Vulnerability to Disease Scale (PVD; Duncan et al., 2009), a 15-item scale with two subscales: Germ aversion and Perceived Infectability. The Germ Aversion facet of the PVD was used as a proxy of pathogen-avoidance motivation in our models ${ }^{3}$. The eight germ aversion items measure a respondent's aversion towards pathogen-related stimuli (e.g. "I prefer to wash my hands pretty soon after shaking someone's hand."). All items were measured on a 7 -point Likert scale (1=absolutely disagree, $7=$ absolutely agree).

Intention and Attitude. Both intentions and attitudes were measured conditionally, that is, participant were asked to rate questions pertaining to these constructs hypothetically, i.e. 'if they would have had unsafe sex in the next week'. We used three items to assess inclination towards testing for STI, each of which were measured on 7-point Likert scales ( 1 =very unlikely, $7=$ very likely). The three intention items were framed over different time periods: "If I had unsafe sex in the next week, I would take an STD-test within a month"; the italicized time-frame replaced in the other items with three months and half a year, respectively. Attitudes were also measured using three indicator items, again with a 7-point Likert scales. Participants indicated how useful, important, and appropriate they considered getting tested for STI should they have unsafe sex during the next week. 


\section{Procedure}

Approval to conduct this study was obtained from the Ethics Committee of Psychology at Maastricht University. After enrolling in the study, participants were directed to the Qualtrics website (http://www.qualtrics.com), where they were asked to provide informed consent. Participants then answered some demographic questions and some additional questions not pertinent to the current investigation. Next, participants completed the Perceived Vulnerability to Disease scale and the Revised Sociosexuality Orientation Inventory. Participants then indicated their attitude toward and intention to be tested for STI. Finally, participants were thanked for their participation, informed about the study hypotheses, and paid.

\section{Statistical Analysis}

Data were analyzed with a structural equation modeling approach using Mplus software (version 7.11). First, we specified a measurement model for the hypothesized constructs. We subsequently modeled the structural relationships between these latent variables. In addition to estimating path coefficients, we report several fit indices for these models: (a) Chi-square test of model fit, (b) the root mean square error of approximation (RMSEA), (c) a standardized root mean square residual (SRMR), and (d) a comparative fit index (CFI). We interpret model fit as adequate or good when RMSEA $\leq .08$ or $\leq .05$, respectively, and SRMR $\leq .08$, and CFI $\geq .90$ or $\geq .95$, respectively (Hu \& Bentler, 1999).

Measurement model. Indicator items were specified to load on their expected latent variables, that is, the items from the previously validated germ aversion facet (Duncan et al., 2009) and the SOI-R (Penke \& Asendorpf, 2008). The indicator items designed to measure testing attitude $(\mathrm{k}=3)$ and intention $(\mathrm{k}=3)$ were specified to load on intention to test, and attitude towards testing latent variables.

Structural models. The models take as starting point earlier theory based findings on the relationship between germ aversion and sociosexuality. Hence, we modeled a relationship between pathogen-avoidance motivation and history of casual sex. We also modeled effects of pathogen-avoidance on intention to test, via attitude towards STI screening. An additional path was specified between past sexual behavior and attitude towards testing. The mediating effect of attitude between a given background variable on intention is common in the theory of planned behavior, and was thus a priori specified in our models.

\section{Results and Discussion}

To determine the appropriate estimation method for our model, we first examined whether our variables satisfied the assumptions of univariate and multivariate normality. These assumptions were not met for our main outcome variables, attitude and intention, so robust maximum likelihood 
estimation (Yuan-Bentler scaled x2) procedure was used in all subsequent models. There were no missing data in our sample.

We thus used Germ Aversion facet items as indicators of a latent variable for pathogen-avoidance motivation. Analysis of a complete measurement model confirmed our expectations about the underlying factor structure, including latent variables for the germ aversion, sexual behavior, attitude and intention items, $\mathrm{x} 2=191.951$, $\mathrm{df}=111, \mathrm{p}<.001$ (CFI=0.967, RMSEA $=0.043$, SRMR $=0.049$ ). The only post-hoc modification to our measurement model was the inclusion of residual covariances between the three indicator items loading on the intention to test construct. Next, we imposed the predicted structural relations on the model (see Figure 1). Here we specified two paths running from pathogen-avoidance scores to STI testing intention, one path from pathogen-avoidance via attitude towards testing intention. The other path ran from pathogen-avoidance to history of casual sex, and again on intention to test via attitude. We then tested the significance of the indirect effect of pathogen-avoidance on attitudes to test through history of casual sex, using bootstrapped $(n=5000)$ standard errors. This indirect effect through history of casual sex was significant, $b=.035, z$ $=2.196, \mathrm{p}=.028 ; 95 \% \mathrm{BCI}[.004 ; .067]$. Additionally, the indirect effects of pathogen-avoidance on intentions to test for STI (via either history of casual sex or testing attitude) were also significant, $b=.024, z=2.149, p=.032$; 95\% BCI [.002; .045].

These results suggest that pathogen-avoidance motivation might influence health-protective behavior by 1) decreasing overall sexual behavior, i.e., having less casual sexual partners, and 2) by leading to increased attitudes and higher intentions to get tested after an act of unsafe sex. Specifically, the results show that the more pathogen avoidant participants were, the fewer sexual partners they report having. Additionally, higher scores on our pathogen-avoidance measure related both directly and indirectly (via history of casual sex) to more positive attitudes and intentions towards getting tested for STI. These results are in line with previous work on pathogen-avoidance and health behavior. Further, similar to work on state induced pathogen avoidance (Meertens et al., 2013; Tybur et al., 2011), results suggest that individual differences in trait pathogen-avoidance tendency appear to be important for understanding sexual behavior itself, and perhaps more relevant, also prophylactic behavior after an act of unsafe sex. 


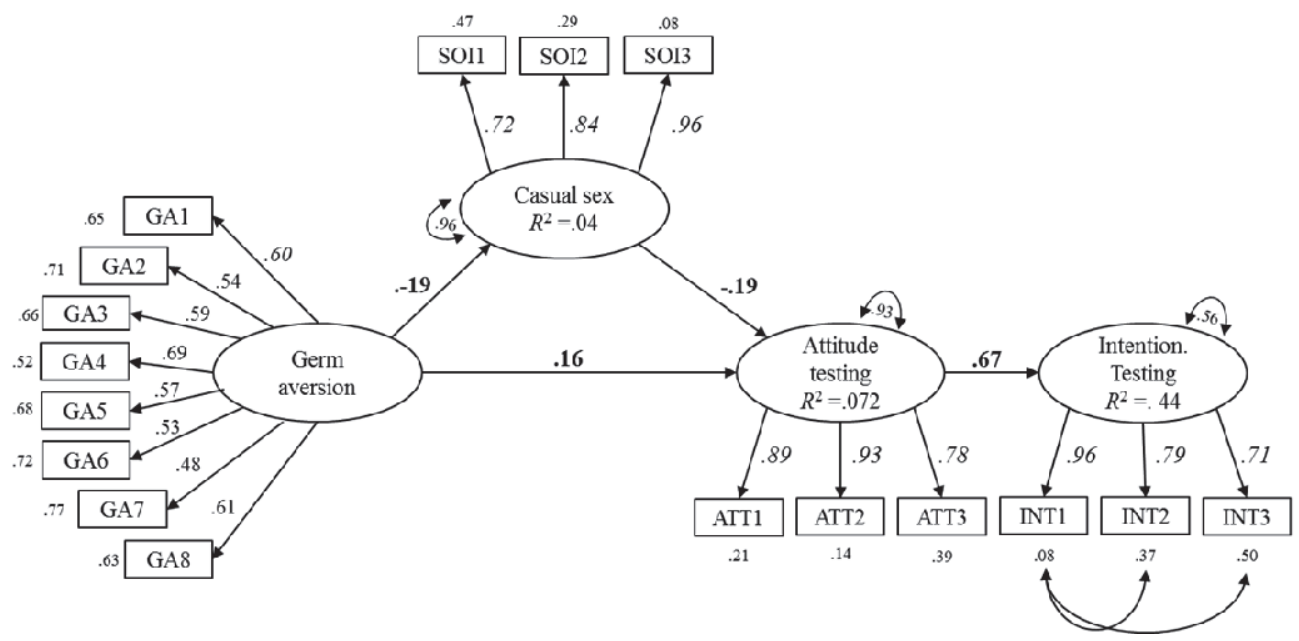

Figure 1. Measurement and structural latent variable model of pathogen-avoidance and STI testing intention. (Study 1). Bold coefficients indicate regression coefficients. $\mathrm{R}^{2}$-values, error terms, and disturbances are provided for the latent variables. All parameter estimates are provided in a fully standardized format (STYX).Italicized coefficients indicate factor loadings. Abbreviations: GA1-GA8 = Germ Aversion indicators; SOI1-3 = Sociosexual behavior indicators; ATT1-3= STI testing attitude indicators; INT1-3 = STI testing intention indicators. Fit indices: $\mathrm{x} 2=192.30, d f=113, \mathrm{p}<.001 ; \mathrm{CFI}=0.968$, RMSEA $=0.043$, SRMR $=0.049$.

\section{Study 2}

Study 1 targeted one specific health-protective behavior: testing for STI. However, it is possible that pathogen-avoidance processes might have broader effects on health-protective behaviors, and not only relate to intention to test for STI. In this study, we therefore examined whether pathogen-avoidance is only important for the regulation of pathogen-related (infectious) disease, or whether it also regulates health-protective behaviors that are not necessarily related to the avoidance of pathogens. Therefore, in Study 2, we aimed to replicate and extend the model from Study 1 by investigating whether pathogen-avoidance relates only to those health behaviors related to infectious disease versus health behaviors for noncontagious diseases.

To this end, in addition to measures of attitude and intention towards getting tested for STI, we included attitudes and intentions toward a variety of testing/screening behaviors related to a broad range of health concerns. We differentiated between pathogen-related screening behavior (e.g., would you get screened for infections after being bitten by a stray dog) and more intention to screen for ostensibly pathogen-unrelated conditions (e.g., would you go to see a doctor if you notice a suspicious mole on your arm). Similar to Study 1, these health behaviors were operationalized with measures of attitude and intention. Moreover, in Study 2, we included an additional, complementary measure of pathogen-avoidance motivation, the pathogen 
disgust facet from the Three Domain Disgust Scale (Tybur et al., 2009). Our hypotheses for Study 2 were as follows:

1) Pathogen-related and pathogen-unrelated screening attitude and intention constructs are distinct, as evidenced by a confirmatory higher-order factor analysis.

2) History of casual sex relates to pathogen-related attitudes and intentions only, but not to pathogen-unrelated screening attitudes and intentions.

3) Pathogen-avoidance motivation directly influences attitudes towards health screening behavior, and especially those behaviors where the health risk relates to pathogens contagion.

4) The relationship between pathogen-avoidance and healthprotective attitudes and intentions is mediated by history of casual sex.

\section{Method}

\section{Participants and selection procedure}

As in Study 1, we used Amazon Mechanical Turk to recruit participants. Selection criteria were identical to Study 1. A total of 627 complete responses to the survey were recorded. After exclusion of non-single participants $(n=119)$, and participants that did not meet our age criterion $(n=38)$, our sample contained a total of $N=470$ participants, including $n=236$ men $($ Mage $=23.4)$ and $n=234$ women $($ Mage= 22.6).

\section{Materials and Measures}

Attitudes and Intentions. Participants' attitudes and intentions were assessed in a manner similar to that employed in Study 1, with three items used as indicators for each health issue. Participants were asked to rate their attitudes towards the following pathogen-related health concerns: 1) "If I had unsafe sex, I think testing for STI would be..." 2) "If I had been bitten by a stray dog, I think testing for rabies would be...", 3) "If I heard on the local news that the flu was going around, I think getting a flu shot would be...". Each measure used three (7-point) semantic differentials (useful, important, appropriate) to assess these attitudes.

Participants also rated attitudes towards testing for the following ostensibly pathogen-unrelated medical conditions: 1) "If I had noticed a new, unusual looking mole, taking a test for skin cancer would be...", 2) "If I felt constantly tired for over a month, getting tested for blood disorders (e.g. an iron-deficiency) would be...", and 3) "If I had repeated, painful headaches for over a week, going to see a doctor for it would be...". The intention questions of all six behaviors were phrased similarly to Study 1 as well, but with timeframes 'within the next week', 'within the next month', and 'within the next three months'.

Pathogen-avoidance. Identical to Study 1, we included the PVD scale. We also included the pathogen disgust facet of the TDDS (Tybur et al., 2009). 
For this instrument, participants were asked to indicate how disgusted they find seven items (e.g., Stepping in dog poop) on a 0 (not at all disgusting) to 6 (extremely disgusting) scale. In this study, we operationalized pathogenavoidance motivation using both the germ aversion scale and the pathogen disgust facet.

\section{Procedure}

The design was largely similar to that of Study 1, except that we included six distinctive health-protective behaviors, three of which related to pathogenic diseases, and three of which related to non-pathogenic health issues. The question groups pertaining to each of these behaviors were presented in a random order. Moreover, to prevent unwanted priming effects of our pathogen-avoidance measures on the dependent variables, participants first filled out questions pertaining to attitude and intention, followed by the pathogen-avoidance motivation measures. At the end of the survey, we asked participants whether they were familiar with the health problems we asked about (rabies, iron-deficiency, e.g.), and scores were replaced with missing values if participants indicated not being familiar with the meaning of a health issue. Missing data for the variables were all under ten percent.

\section{Statistical Analysis}

Our structural equation modelling approach was the same as previously reported. First, we conducted CFA's to determine the validity of the scales used in this study. Given that we had missing data (maximum of $10 \%$, and our variables were skewed, we opted to use a robust maximumlikelihood estimation (Yuan-Bentler scaled chi-square). Because we had two convergent measures of pathogen-avoidance motivations in this study, we specified a higher-order factor ('pathogen-avoidance') and tested whether both measured a similar high-order construct. Given that we included six distinctive behaviors (pathogen-related versus pathogen-unrelated) we also specified another two higher-order factors in our measurement model for attitudes, 1) a pathogen-related attitudes factor, using the STI, dog bite, and flu shot constructs as indicators, and 2) a pathogen-unrelated factor, using the mole, iron-deficiency, and headache constructs. The same hierarchical structure in the factors was specified for intentions to test. First, we compared a discriminant model (first-order factors arranged under two higher-order factors: pathogen-related versus pathogen-unrelated variables) with a congeneric model (all behaviors loading on one 2nd order construct). We then proceeded in two steps, first by replicating our previously reported model, and second by extending the structural model with the additional health-protective behaviors. Finally, we tested the indirect effects in our structural model using bootstrapped $(n=5000)$ standard errors to construct 95\% confidence intervals. 
Table 1. Model fit statistics for the higher-order confirmatory factor analyses (Study 2)

\begin{tabular}{llllllllll}
\hline Measure & Model & ${ }^{a}$ Obs & YB- X $^{2}$ & df & CFI & RMSEA & 90\% CI & SRMR & Cov \\
\hline Attitudes & congeneric & 18 & $259.21^{*}$ & 126 & 0.963 & 0.047 & {$[0.039 ; 0.056]$} & 0.039 & 3 \\
& discriminant & 18 & $258.35^{*}$ & 125 & 0.963 & 0.048 & {$[0.039 ; 0.056]$} & 0.038 & 3 \\
Intentions & congeneric & 18 & $367.94^{*}$ & 119 & 0.933 & 0.067 & {$[0.059 ; 0.075]$} & 0.068 & 10 \\
& discriminant & 18 & $361.79^{*}$ & 118 & 0.934 & 0.066 & {$[0.059 ; 0.074]$} & 0.066 & 10 \\
Casual sex & SOI-B & 3 & $16.45^{*}$ & 2 & 0.948 & 0.124 & {$[0.073 ; 0.182]$} & 0.049 & 0 \\
PA & GA, PD & 15 & $207.93^{*}$ & 86 & 0.900 & 0.055 & {$[0.045 ; 0.064]$} & 0.057 & 3 \\
All & complete model 54 & $2497.46^{*}$ & 1328 & 0.918 & 0.043 & {$[0.041 ; 0.046]$} & 0.071 & 29 \\
\hline
\end{tabular}

Note. $\mathrm{PA}=$ pathogen-avoidance; GA= Germ aversion scale; $\mathrm{PD}=$ =Pathogen disgust scale; SOI-B = Sociosexual orientation behavior facet. a observed variable count. Yuan-Bentler rescaled $\mathrm{X}^{2}$; $\mathrm{df}=$ model degrees of freedom; $\mathrm{CFI}=$ comparative fit index; RMSEA= root-mean squared error of approximation; $\mathrm{SRMR}=$ standardized root mean squared residual; $\mathrm{COV}=$ residual covariances in model. ${ }^{*} \mathrm{p}<.01$

\section{Results and Discussion}

\section{Measurement model}

The results of our CFA analyses, including a comparison of congeneric versus discriminant structures for attitudes and intentions are reported in Table 1. Although both congeneric as discriminant factor structures fit equally well, we opted to proceed to test structural relationships by maintaining a distinction between pathogen-related and pathogen-unrelated conditions. The germ aversion and pathogen disgust scales loaded strongly on a common pathogen-avoidance factor, suggested by high factor loadings on the 2nd-order factor. The overall measurement model fit was acceptable (see Table 2 for correlations between the constructs).

\section{Structural model}

We specified an initial model to replicate the relationship between pathogen-avoidance and attitude/intention towards getting screened for STI after an act of unsafe sex. This model was conceptually identical to the one reported in Study 1. Results indicate that our previously reported model replicates well in this sample.

Table 2. Observed correlation matrix for the full-SEM model (Study 2)

\begin{tabular}{lllllllll}
\hline Measure & 1 & 2 & 3 & 4 & 5 & 6 & 7 & 8 \\
\hline 1. Germ aversion & --- & & & & & & & \\
2. Pathogen Disgust & .56 & --- & & & & & & \\
3. Sexual behavior & -.14 & -.16 & --- & & & & & \\
4. Intention PR & .26 & .29 & -.22 & --- & & & & \\
5. Intention PU & .19 & .21 & -.12 & .92 & --- & & & \\
6. Attitudes PR & .35 & .38 & -.29 & .76 & .66 & --- & & \\
7. Attitudes PU & .28 & .31 & -.17 & .74 & .68 & .97 & --- & \\
8. PA & .72 & .79 & -.20 & .36 & .26 & .48 & .39 & --- \\
\hline
\end{tabular}

Note. All measures except PA represent first-order constructs. For the attitudes and intention constructs only 2nd-order factors are correlated. Abbreviations: $\mathrm{PA}=$ pathogen-avoidance; $\mathrm{PR}=$ Pathogen-related; $\mathrm{PU}=$ Pathogen-unrelated . 
Table 3. Bootstrapped mediation effects on intentions (Study 2)

\begin{tabular}{llllll}
\hline Outcome & Indirect Effect via & $\mathrm{b}$ & $\mathrm{Z}$ & $\mathrm{p}$ & $95 \% \mathrm{CI}$ \\
\hline Intention PR & Attitudes PR & .33 & 4.31 & .00 & {$[.18 ; .48]$} \\
& Attitudes PR - SOI & .03 & 1.79 & .07 & {$[-.00 ; .06]$} \\
& Total effect PR & .36 & 4.59 & .00 & {$[.21 ; .52]$} \\
Intention PU & Attitudes PU & .25 & 3.82 & .00 & {$[.12 ; .38]$} \\
& Attitudes PU - SOI & .01 & 1.26 & .21 & {$[.01 ; .03]$} \\
& Total effect & .26 & 4.00 & .00 & {$[.13 ; .39]$} \\
\hline
\end{tabular}

Note. Abbeviations: $\mathrm{PR}=$ pathogen related testing, $\mathrm{PU}=$ pathogen unrelated, $\mathrm{SOI}=$ Sociosexuality index, CI= Bootstrapped $(n=5000) 95 \%$ confidence interval.

Next, we tested our model including all health-protective behaviors. We specified a model in which pathogen-avoidance predicted sexual behavior and both the pathogen-related and pathogen-unrelated health behaviors (see Figure 3). Attitudes were specified to be the direct predictor of intentions, as described in Study 1. Results indicate that, in accordance with expectations, pathogen-avoidance predicted intention to test for pathogen-related health issues via attitude and also via history of casual sex. Contrary to expectation, however, pathogen-unrelated intentions were predicted equally strong by pathogen-avoidance, again via attitudes and sexual behavior. Notably, though, the indirect effects from pathogenavoidance on intentions through sexual behavior were descriptively stronger for pathogen-related testing intentions. The indirect effects of pathogenavoidance motivations on intentions to test, both pathogen-related as unrelated, were also tested for significance. There were indirect effects through attitudes on both the higher-order intention constructs (see Table 3 ). However, the indirect effects through both sexual behavior and attitudes were not statistically significant.

\section{General Discussion}

The aim of the current research was to investigate how motivations to avoid pathogens relate to health-protective behaviors. Results from Study 1 revealed that participants' intentions to be tested for STI were predicted by pathogen-avoidance variables. Moreover, attitudes toward testing and number of sexual partners mediated the effect of pathogen-avoidance on testing motivations. Study 2 replicated and extended these findings by showing moderate associations between pathogen-avoidance and a broad range of health-protective behaviors, including but not limited to pathogenrelated diseases. In addition, a comparison of the explained variance in pathogen-related versus pathogen-unrelated health-protective intentions (Study 2) indicated that $58 \%$ of the variance in pathogen-related intentions were explained by the variables in our model, versus $46 \%$ of the variance in pathogen-unrelated intentions. Our models also suggest that pathogenavoidance motivations explain unique variance in testing intentions, although a significant amount of the variance in testing intentions was explained through attitudes. 


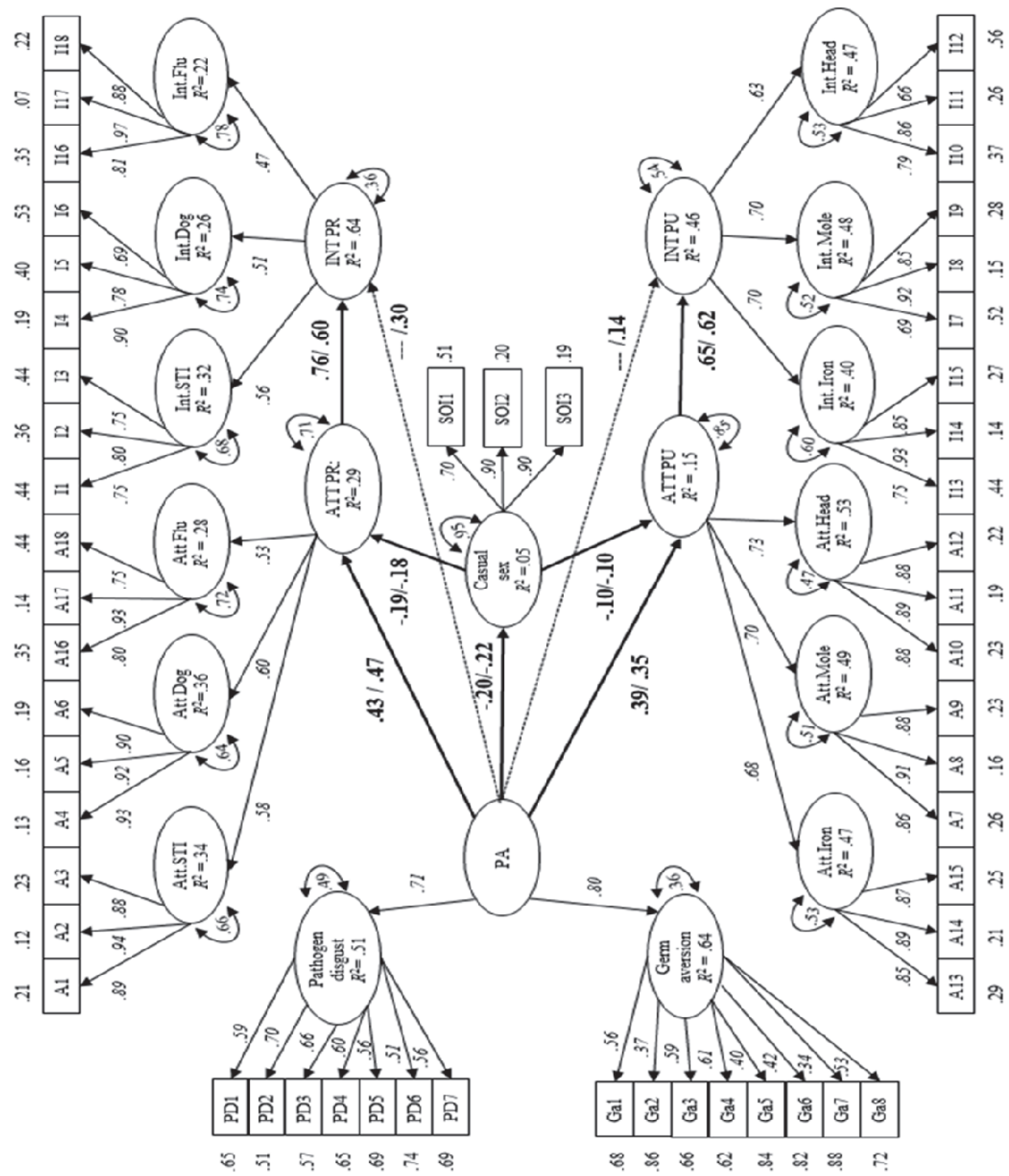

Figure 3. Measurement and structural latent variable model (Study 2). The diagram represents two structural models, the first includes freely estimated direct effects of pathogen avoidance on intention (coefficients right of the slash) and the second in which direct effects are constrained to zero (weights left of the slash). Italicized coefficients indicate factor loadings. Abbreviations: PA $=$ Pathogen-avoidance motivation; Attitudes PR =

Pathogen related testing attitudes; Attitudes PU = Pathogen unrelated attitudes; GA1-GA8 = Germ Aversion Scale; PD1-PD7 = Pathogen disgust scale; SOI1-3 = Sociosexuality behavior scale. Fit indices full model: $\mathrm{x} 2=2502.83^{*}, d f=1332, p<.001 ; \mathrm{CFI}=0.918$, RMSEA $=$ $0.043, \mathrm{SRMR}=0.071$. Fit indices restricted model: $x 2=2515.63^{*}, d f=1334, p<.001$; CFI $=0.917, \mathrm{RMSEA}=0.043, \mathrm{SRMR}=0.073 . \Delta \times 2(2, N=470)=12.80, p=.002$ (Satorra-Bentler scaled). 
The first main finding we reported across two studies, a negative association between pathogen-avoidance and sexual behavior, is in line with previous work. To understand variance in pathogen-avoidance tendencies, researchers (Murray et al., 2013; Mark Schaller \& Park, 2011; Tybur et al., 2013) have considered trade-offs involved in the regulation of sexual behaviors. On the one hand, individuals are motivated to have sex, often in the absence of a monogamous relationship. On the other hand, sexual intercourse is a major risk factor for pathogen transmission, and some of the implicit sexual avoidance motivations might have roots in pathogenavoidance motives. This interplay between competing motives is illustrated in our studies: participants who are more pathogen-avoidant had a history of fewer different sex-partners. Thus, the results suggest that differences in sexual behavior might be partly linked to pathogen-avoidance tendencies.

Our results further suggest that pathogen-avoidance motivations have two complementary health-protective effects: first, by reducing the inclination towards casual sex, and second, by increasing attitudes and intentions towards health screening behavior. Interestingly, higher levels of pathogen-avoidance tendencies were strongly linked to increased attitudes and intentions towards testing for STI, as well as to a range of both pathogen-related and unrelated screening behaviors. This implies that individuals with stronger motivations to avoid pathogens are more protective of their health in general.

Initially, it is not straightforward to assume that high levels of pathogen-avoidance should be associated with more positive evaluations toward getting screened for concerns such as a suspicious mole or a constant headache. Yet, our results speak for a generalized relationship between pathogen-avoidance and health-protective behaviors - although a substantially smaller percentage of variance was explained in pathogenunrelated testing intentions. Although these results were not completely in line with our hypotheses, they do not necessarily argue against the view that the behavioral immune system is specialized for managing the threat of pathogen transmission. Rather, the generalized tendency to get screened whether the health concern relates to pathogens or not - might indicate that pathogen-avoidance motives guide decisions about health even for conditions not directly associable with pathogens (Mark Schaller \& Park, 2011). Thus, results here may suggest that individuals with higher pathogen avoidance motivation are biased to treat symptoms potentially indicative of contagion - whether this be a frequent headache, a mole, a dog bite, or unsafe sexual behavior - as valid and worthy of treatment. In short, variation in pathogen-avoidance seems to relate to a generalized tendency to act more health-protectively, suggesting that pathogen-avoidant individuals might worry more about their health in general.

Our analysis of mediated effects of pathogen-avoidance on testing intentions also indicated an indirect effect through past sexual behavior on attitudes towards testing for pathogen-related disease (this approached 
significance), but the indirect effect towards pathogen-unrelated conditions was descriptively smaller. The negative relationship between history of casual sex and testing attitudes may possibly reflect decreased risk perceptions in these participants, although the current design may not allow further conclusions about the direction of causation in these correlations. The understanding that sexual health behavior might come about by an interplay between competing motives for behavior could be used to optimize health-promotion strategies in the domain of sexual health. The motivational systems related to mating and pathogen-avoidance might antagonistically influence sexual behavior, either temporarily, as illustrated in research by Borg and De Jong (2012) or more chronically, as the observed association between trait pathogen-avoidance and sexual behavior in the current studies seems to suggest (see also Duncan et al., 2009; Murray et al., 2013). Mating motives might 'overrule' the dispositional as well as the temporarily activated behavioral immune system, and hence might reduce health concerns associated with sexual behaviors. Conversely, as shown by our current results, high levels of pathogen-avoidance motives may reduce individuals' interest in casual sex, and increase prophylactic behavior.

How might up-regulation of pathogen-avoidance motivation - and thus increases in health-protective behavior - be achieved by health promoters? One way health promotion research can benefit from knowledge of such evolved motives - including those relating to pathogen-avoidance - is to place emphasis on the context in which the health behavior occurs, and to examine which contextual cues might trigger relevant motivational states that could bolster health-protective behavior (Meertens et al., 2013). Although fear has often been a targeted emotion in health promotion research - with tentative effectiveness (Peters et al., 2013), our current results suggest that the emotion disgust (as an important motivational state driving pathogenavoidance) might be a more effective health promoting emotion.

\section{Limitations and directions for future research}

The study sample in the current research has a restricted age-range (18-27) with a large variance in sexual behavior; it thus remains to be tested whether the observed relationships between the variables are similar in populations with other demographic characteristics. Moreover, the current cross-sectional design does not allow causal tests of the model we postulated, and thus further work is needed to test the model presented here. Given that our pathogen-avoidance measures reflect a temporal stable trait (cf. B. O. Olatunji et al., 2012), we chose this variable as the most antecedent variable in our current models. In our view, both sociosexuality (casual sex history) and future health-protective behavior can partly be understood by investigating pathogen-avoidance motivations. The validity of this model remains to be tested in follow-up research.

A further limitation of the current study is that the outcome variables currently used (intention to perform a behavior) do not perfectly align with 
actual behavior (T. L. Webb \& Sheeran, 2006). Conclusions that pathogenavoidance processes influence actual health-protective behavior are therefore tentative. Follow-up work is needed to bridge a possible intentionbehavior gap, and to examine the relationship between pathogen-avoidance motivation and actual health-protective behavior. Future work could also address how the psychology of pathogen-avoidance relates to the participants' behavioral beliefs about disease contraction.

Overall, our results show how pathogen-avoidance processes may provide a new angle for understanding health-protective behaviors, and might complement existing health psychology approaches in the prediction and changing of health behavior. With an eye on health promotion, understanding when and how pathogen-avoidance motivation affects healthprotective behavior might contribute to the development of effective interventions. And, more generally, further exploration of fundamental motivational systems, like those pertaining to pathogen-avoidance, may provide novel and promising tactics to increase intervention effectiveness. 

Chapter 4: Attitude in a motivational context: THE EFFECT OF ELICITED PATHOGEN-AVOIDANCE MOTIVATION ON SCREENING ATTITUDE AND BELIEF IMPORTANCE

This chapter is based on: Gruijters, S.L.K., Ruiter, R.A.C., Massar, K., \& Tybur, J.M. (under review). Attitude in a motivational context: The effect of elicited pathogen-avoidance motivation on screening attitude and belief importance. 


\begin{abstract}
Beliefs are central to the formation of attitudes towards testing for sexually-transmitted infections (STI). But is the importance of specific beliefs dependent on state-level motivational factors? This study sought to examine how belief importance varies between individuals depending on their motivational state. Using evolutionary psychological frameworks of motivation, we hypothesized that inducing a motivation to avoid pathogens would alter attitudes towards STI testing, and impact the importance of specific beliefs. In a belief-elicitation pilot study $(n=30)$, eight modally salient beliefs were selected. Four of which directly involved disease-related outcomes of STI-testing, and four others pertained to social outcomes. In a subsequent online randomized controlled trial $(N=270)$, participants were either exposed to an essay designed to trigger pathogen disgust or to an essay with a neutral context. We subsequently made a between-group comparison of a latent variable model of attitude, to test the hypothesis that the predictive strength of specific expectancy-value components would be affected by triggering pathogen-avoidance motivation. Results partially supported our hypothesis, showing significant differences in belief importance between-groups. However, the pathogen-avoidance manipulation failed to induce changes in STI testing attitude. We conclude that state-level motivations may contribute to understanding which beliefs shape individuals' attitude towards STI-testing.
\end{abstract}

Keywords: pathogen-avoidance, attitude, STI-testing, beliefs, expectancy-value, MIMIC model 


\section{Introduction}

For many health psychologists, the primary goal of basic behavioral research is to convert empirical findings and theoretical insights into targeted interventions. The design of successful interventions thus rests on the availability of a wide and substantial theory-base (cf. Michie \& Johnston, 2012). Thus far, the most widely adopted behavior change models used by health psychologists have not incorporated insights from evolutionary perspectives (Gruijters, Tybur, Massar, \& Ruiter, under review), which focus on the ultimate causes of behavior (Scott-Phillips et al., 2011). Such an evolutionary perspective might potentially offer new insights into health behavior and underlying determinants, and the knowledge gained by such an approach might practically extend into the development of novel intervention strategies (Gruijters et al., 2016; Tybur et al., 2012).

One model recently developed by evolutionary social psychologists, specifying the evolved, functional domains of motivated behavior, could be fruitfully applied to understand health behavior. The Fundamental Motives Framework (Kenrick, Griskevicius, et al., 2010; Kenrick, Neuberg, et al., 2010) posits a number of threats and affordances that are expected to steer motivated behavior, and which might be used to inform understanding of health behavior as well. The central idea behind this model is that the recurring problems ancestral humans faced, such as successfully rearing offspring, forming coalitions in groups, and avoiding the contraction of disease can make predictions about the discrete goals and needs underlying motivated behavior.

In the current paper, we aim to mesh the Fundamental Motives framework with approaches often used to predict health behavior: the Theory of Planned Behavior (Ajzen, 1985) and its successor the Reasoned Action Approach (Fishbein \& Ajzen, 2010). By doing so, we examine how considerations of fundamental motivations for behavior can add to the predictive power of belief-based or social cognitive models. Specifically, we focus our examination of these models on young adults' attitude to get tested for sexually-transmitted infections (STI).

According to the TPB (Ajzen, 1985; Fishbein \& Ajzen, 2010) the most proximal predictor of behavior is the intention to perform a behavior, referred to as behavioral intention (see for a meta-analysis; Webb \& Sheeran, 2006). The strength of a given behavioral intention is in turn determined by attitudes, perceived social norm, and perceived behavioral control (see for a metaanalysis; Sheeran et al., 2016). The model further postulates that these determinants are shaped by a set of beliefs, specifically those that are readily available to a person in a specific context. Behavioral beliefs constitute an individual's conceptions about the consequences of a particular behavior, and consist of both negative and positive beliefs about that behavior. For example, a negative outcome expectation related to risky sexual behavior may be that 'sex without a condom puts one at risk for sexually-transmitted diseases', versus a positive outcome that 'sex without a condom is more enjoyable'. 
In the TPB, the manner in which a set of (available) beliefs determines a particular attitude towards a behavior is usually understood using an expectancy-value model, in which the outcome of a behavior is weighted by the subjective probability of the behavioral outcome. For example, STI testing might lead to knowledge of one's infection status. This outcome can be evaluated on desirability (or value), for instance is knowledge of infection status something that is desirable? But also on the likelihood the behavior leads to this outcome (the expectancy component), that is, is getting tested likely to inform about infection status? TPB approaches assume that summation of the desirability and likelihood product ratings of salient behavior outcomes determines an attitude towards a behavior. Similar belief structures are thought to determine the perceived social norm and behavioral control variables in this model. In sum, the TPB provides a framework of the psychological variables important for understanding when a particular behavior is likely to be performed.

However, the TPB does not straightforwardly provide an explanation for a key issue: variance in belief importance, that is, that the importance of specific beliefs varies across individuals. Van der Pligt and De Vries (1998) provided initial evidence that models incorporating belief importance were better able to predict an individual's attitude towards smoking, compared to using only an expectancy-value approach to attitude formation. Their method of tapping into the importance of beliefs is based on the idea of dimensional salience (see Newton, Ewing, Burney, \& Hay, 2012; Newton, Newton, \& Ewing, 2014a; van der Pligt \& de Vries, 1998), which requires participants to nominate the three - or five (e.g., Harreveld, Pligt, Vries, \& Andreas, 2000) - most important behavioral beliefs about the consequences of a specific behavior.

Consider for example two behavioral beliefs - one negative and one positive - about the consequences of smoking: "[smoking] fosters social intercourse" and "[smoking is] bad for one's health". Applying an expectancy-value approach, Van der Pligt and de Vries (1998) showed that the overall outcome evaluation differed between smokers and non-smokers, although both groups evaluated the 'bad health' outcome as a negative one and the 'social intercourse' outcome as positive. However, the groups differed with regard to the importance they assigned to each of these beliefs: the social (desirable) outcome was more frequently ranked as important by smokers than by non-smokers. Moreover, this importance differences was substantially larger than the overall outcome evaluation difference between smokers and nonsmokers. Conversely, the undesirable outcome of smoking (bad for one's health) was ranked as more important by the non-smokers. Additionally, Harreveld, Van der Pligt, De Vries and Andreas (2000) found evidence that important attributes were more readily retrieved and evaluated compared to those considered of lesser importance.

These results illustrate that when applying an expectancy-value approach, the importance of a behavioral belief need not closely align with 
the overall outcome evaluations, may differ between individuals, and add a different dimension to understanding the formation of attitudes towards behavior. However, although the dimensional salience approach may provide a more comprehensive descriptive account of attitude formation, it does not provide an answer to one open question: Why are some beliefs more important to some individuals than they are to others? Here, we attempt to examine this issue using the fundamental motives framework (Kenrick, Griskevicius, et al., 2010; Kenrick, Neuberg, et al., 2010; Neuberg et al., 2011). This framework might further provide insight into differences between individuals, but also across contexts, in the importance of behavioral beliefs as predictors of attitude.

\section{Pathogen-avoidance motivation}

According to the fundamental motives framework, our basic motivational systems evolved over time in response to various recurring problems our ancestors faced, among which are self-protection, pathogen-avoidance, mate acquisition, mate retention, social affiliation, parenting (kin-rearing), status, and resource acquisition (Kenrick, Griskevicius, et al., 2010; Kenrick, Neuberg, et al., 2010; Neuberg et al., 2011). One motivational system evolved to manage specific disease-threats relates to mechanisms regulating pathogen-avoidance (Tybur \& Lieberman, 2016), and this motivational state is closely tied to the emotion disgust (Tybur et al., 2013). A few studies have shown the potential of focusing on such motivations for understanding health behavior. For example, Tybur, Bryan, Magnan and Hooper (2011) devised an experiment in which they aimed to activate a pathogen-avoidance motive by using an olfactory cue to pathogens. Before participants entered the lab, the experimenter sprayed a single-dose of a spray resembling the smell of feces. This implicit cue to pathogens caused participants to indicate an increased willingness to use condoms in future sexual encounters, buy and carry condoms with them, and discuss condom-use with future sex partners. Moreover, such environmental influences on health-protective behavior were also examined in a study by Meertens, Branković, Ruiter, Lohstroh, and Schaalma (2013). Participants were asked to imagine that they went home with someone after a night out, had unsafe sex, and the next morning either woke up in a tidy room (control condition) or a very sloppy, even dirty room. Next, they were asked to rate the likelihood of having contracted a sexuallytransmitted infection, and to indicate the probability of engaging in this behavior again. Results supported the researchers' hypotheses, and indicated that participants in the dirty environment condition - a cue to the possible presence of pathogens - felt more susceptible to having contracted a sexuallytransmitted disease, and were less inclined to repeat such behavior in the future. More recently, Gruijters, et al. (2016) also observed that motivations to avoid disease contraction (measured using trait pathogen disgust indices) predicted various health-protective attitudes and intentions, including those pertaining to getting tested for STI. 
These studies have in common that the conditions of the environment motivated disease-avoidant behaviors. Within the TPB framework these results are interpreted to indicate that the experimental manipulations changed the determinants of behavior - i.e., attitudes, social norm, and perceived behavioral control). Considering the nature of the manipulations (olfactory and visual cues to pathogens) we suggest that it is likely that these manipulations temporarily activated a motive for pathogen-avoidance, which in turn affects behavioral determinants. This process should therefore be reflected in the underlying beliefs - either directly in expectancy-value components or in their relative importance.

In the current study we therefore examined how temporarily activating a pathogen-avoidance motive might influence the relative importance - or temporary salience - attached to specific behavioral beliefs that predict participants' attitude towards STI testing. Thus, we were interested to examine how the relative importance of behavioral beliefs that form the attitude towards STI testing is affected by the activation of a pathogen-avoidance motive. Relative belief importance is operationalized in this study by inspecting the size of the respective regression coefficient of the expectancyvalue product terms on the latent attitude variable. This regression weight comparison approach is an alternative to having participants select the most important beliefs (e.g. select the 5 most important) used in earlier belief importance research (Harreveld et al., 2000; Pligt \& De Vries, 1998; van der Pligt et al., 1998), and allows a sensitive comparison of group differences.

First, we conducted a belief elicitation (pilot) study to be able to select a total of eight beliefs about STI testing behavior. Four of these related directly to disease contraction, and four behavioral outcomes of STI testing did not pertain to disease-related outcomes of STI testing. Both expectancy (E) and value (V) components of these behavioral beliefs were measured and their products were used to predict attitude towards STI testing, in line with expectancy-value theory. We hypothesized that inducing a pathogenavoidance motivation would (1) positively affect the relevance of behavioral outcomes associated with disease, and (2) decrease the importance of those outcomes not related to disease. The full theoretical model and predictions will be presented in the Method section.

\section{Method}

\section{Participants, selection procedure and design}

Using a crowdsourcing platform, Prolific Academic (https://prolific.ac; see Peer, Brandimarte, Samat, \& Acquisti, 2017) participants were invited via an advertisement to participate in the current study. We limited our recruitment to men and women aged 18-27 because this population is expected to show the largest variance in sexual behavior overall. Our sample consisted of $60.4 \%$ British residents and $18.1 \%$ from the United States. The remaining percentage consisted of participants of a variety of mostly European countries. A total of 324 responses to the survey were recorded. 
Participants who responded to our survey were randomly assigned to one of two between-subjects conditions (Condition: Experimental / Control). In the experimental condition $(n=132)$ participants read a text that has been shown to successfully evoke pathogen-avoidance motivation (White, Kenrick, $\&$ Neuberg, 2013). Participants in the control condition $(n=138)$ received a non-priming story about a person that had lost his keys. Three control questions, assessing knowledge of the essay content, were added to the design to ensure that participants had read the text. We used the responses to these control questions as an inclusion criterion for both the experimental and control condition.

After excluding non-complete responses $(n=13)$, participants that failed to correctly answer the integrated control questions $(n=37)$, and those that did not provide informed consent $(n=4)$, the final sample consisted of $N=270$ participants; 146 males $\left(M_{\text {age }}=22.0, S D=3.02\right)$ and 117 females $\left(M_{\text {age }}=22.4\right.$, $S D=2.70)$. A total of 153 participants (56.6\%) indicated to be involved in a romantic relationship.

\section{Materials and Measures}

Belief selection pilot study. A belief elicitation study $(N=30)$ about STI testing was conducted in line with the procedure suggested by Fishbein and Ajzen (2010). While we inquired about behavioral, normative and control beliefs, this study only used the elicited behavioral beliefs listed by participants. The questions were open-ended and asked participants to "Please take a few minutes to tell us what you think about the possibility of getting tested for sexually-transmitted diseases, after having had unsafe sex. There are no right or wrong responses, we are merely interested in your personal opinions. In response to the questions below, please list the thoughts that come immediately to mind. Write each thought on a separate line." Subsequently, the following questions were posed: (1) What do you see as the advantages of getting tested for sexually-transmitted diseases after having had unsafe sex?; (2) What do you see as the advantages of not getting tested for sexually-transmitted diseases after having had unsafe sex?; (3) What do you see as the disadvantages of getting tested for sexually-transmitted diseases after having had unsafe sex?; (4) What do you see as the disadvantages of not getting tested for sexually-transmitted diseases after having had unsafe sex?; and (5) What else comes to mind when you think about getting tested for sexually-transmitted diseases after having had unsafe sex?

Pathogen-avoidance manipulation. The experimental manipulation consisted of a short text (approx. 830 word length). The text asked participants to imagine being a student making a visit to a geriatric ward. The text vividly described events that through eliciting disgust responses has been shown to evoke a pathogen-avoidance motive (White et al., 2013). An example section of the text reads: "A couple weeks ago you had to dissect a pig preserved in a foul-smelling formaldehyde solution. Next week's assignment is volunteer work in the geriatric ward of a local hospital. You 
recall visiting your great-grandmother in the hospital, and remember how the sight and smell of all those elderly patients made you feel a bit queasy." The control text described a neutral situation in which a person had lost her/his keys, and was matched in number of words to the pathogenavoidance text (see White et al., 2013).

Control questions. To increase the power of our statistical analyses (Oppenheimer, Meyvis, \& Davidenko, 2009), we added attention check questions to the survey structure. These questions were provided in multiple-choice format (3-choice) and were not difficult to answer if participants read the text completely. Participants were asked factual questions about the experimental manipulation. For the experimental condition these were: 1) 'In the text, the character is given the advice to take a short break, by whom?', 2) 'The activity in the story was part of a school course, which course was it?' , 3) 'In the story, the character's first task was...'. The control group questions were: 1) 'In the story, some keys had gone missing. Whose keys were they?', 2) 'In the story, the main character has fed some pets, what kind of pets?', 3) 'In the story, the character was doing something to relax, what was it?'.

Expectancy-Value scores as predictors of attitude towards STI testing. Based on the belief elicitation study, we selected four outcomes of STI testing that relate directly to pathogen-avoidance motivation (See Table 1, EV1-EV4), and four outcomes that did not directly relate to pathogenavoidance motivation (See Table 1, EV5-EV8). EV5-EV8 were chosen because they did not pertain to disease related outcomes - these instead related to social motivations and effort involved in getting tested for STI. The behavioral outcomes were assessed on their desirability ( 1 =bad, $7=$ good) and likelihood (1=unlikely, $7=$ likely), and the responses for the desirability ratings of items V4, V5 and V6 were reverse-coded to allow a meaningful computation of the product terms. For both the disease-related as well as the disease-unrelated beliefs, expectancy-value scores were created by computing products of the expectancy scores multiplied by the outcome evaluation $\left(\mathrm{E}^{\mathrm{i} *} \mathrm{~V}\right.$ i). Each product-variable was used as predictor of attitude towards STI testing. This is conceptually equivalent to expectancy-value theory, which states that a behavioral attitude is formed by the additive effects of expectancy-value products of salient beliefs. 
Table 1. Behavioral beliefs measured in the study, both an expectancy and value component were assessed.

\begin{tabular}{|c|c|c|c|}
\hline Group & Item & Mean & $\mathrm{SD}$ \\
\hline \multicolumn{4}{|c|}{ Disease related beliefs } \\
\hline E1 & $\begin{array}{l}\text { [If I had unprotected sex with a stranger], a test for } \\
\text { sexually-transmitted diseases (STIs) would accurately tell } \\
\text { me whether I had contracted an STI. }\end{array}$ & 6.15 & 0.964 \\
\hline V1 & For me, knowing the results of an STI test would be & 6.36 & 1.151 \\
\hline E2 & $\begin{array}{l}{[\ldots] \text {, getting tested for sexually-transmitted diseases (STIs) }} \\
\text { would result in me getting early treatment that could } \\
\text { prevent negative long-term health consequences. }\end{array}$ & 6.59 & 0.770 \\
\hline V2 & $\begin{array}{l}\text { My getting early treatment for sexually-transmitted } \\
\text { diseases (STIs) that can prevent long-term health } \\
\text { consequences would be }\end{array}$ & 6.77 & 0.757 \\
\hline E3 & $\begin{array}{l}{[\ldots] \text {,getting tested for sexually-transmitted diseases (STIs) }} \\
\text { would reduce my feelings of anxiety/fear of having } \\
\text { contracted an STI }\end{array}$ & 5.96 & 1.510 \\
\hline V3 & $\begin{array}{l}\text { My being able to reduce anxiety/fear of having contracted } \\
\text { an STI would be }\end{array}$ & 6.40 & 1.165 \\
\hline E4 & $\begin{array}{l}{[\ldots], \text { getting tested for sexually-transmitted diseases (STIs) }} \\
\text { would cause stress and worry due to the possibility of } \\
\text { testing positive }\end{array}$ & 5.60 & 1.329 \\
\hline V4 & $\begin{array}{l}\text { Experiencing stress and worry over the possibility of a } \\
\text { positive STI test result would be (R) }\end{array}$ & 5.63 & 1.386 \\
\hline \multicolumn{4}{|c|}{ Disease unrelated beliefs } \\
\hline E5 & $\begin{array}{l}{[\ldots] \text {,getting tested for sexually-transmitted diseases (STIs) }} \\
\text { would be embarrassing for me }\end{array}$ & 4.20 & 2.010 \\
\hline V5 & Being embarrassed about getting an STI test would be (R) & 5.46 & 1.334 \\
\hline E6 & $\begin{array}{l}{[\ldots] \text {,getting tested for sexually-transmitted diseases (STIs) }} \\
\text { would require me to invest time and effort to visit a doctor } \\
\text { or clinic. }\end{array}$ & 5.82 & 1.217 \\
\hline V6 & $\begin{array}{l}\text { Having to invest time and effort to visit a doctor/clinic } \\
\text { would be }(R)\end{array}$ & 4.00 & 1.765 \\
\hline E7 & $\begin{array}{l}{[\ldots] \text {,getting tested for sexually-transmitted diseases (STIs) }} \\
\text { would prevent me from passing on a possible infection to } \\
\text { future sexual partners. }\end{array}$ & 6.13 & 1.347 \\
\hline V7 & $\begin{array}{l}\text { Preventing passing on an infection to future sex-partners } \\
\text { would be }\end{array}$ & 6.50 & 1.480 \\
\hline E8 & $\begin{array}{l}{[\ldots] \text {,getting tested for sexually-transmitted diseases (STIs) }} \\
\text { would enable me to inform past sexual partners about a } \\
\text { possible infection. }\end{array}$ & 6.03 & 1.341 \\
\hline V8 & $\begin{array}{l}\text { Being able to inform past sex-partners about a possible } \\
\text { infection would be }\end{array}$ & 5.84 & 1.534 \\
\hline
\end{tabular}

Note. $\mathrm{E}=$ expectancy (likelihood) rating of an outcome, $\mathrm{V}=$ value (desirability) rating of outcome. All expectancy items were rated on a 1 (unlikely) to 7 (likely) scale. $\mathrm{R}=$ reversed coded

Direct measure of attitude towards STI testing. We used five items as a direct measure of attitude towards testing for STIs, each of which measured on 7-point Likert scales. The five items were "If I had unprotected sex with a stranger, getting tested for sexually-transmitted diseases (STIs) would be very... - 1) useful / useless, 2) good / bad, 3) important / unimportant, 4) appropriate / inappropriate, and 5) beneficial / unbeneficial." 


\section{Procedure}

Approval to conduct this study was obtained from the Ethical Review Board Psychology at Maastricht University. The study was advertised on Prolific Academic (http://prolific.ac.uk) as a study into the 'determinants of STI testing'. After enrolling, participants were directed to the survey, where they were asked to provide informed consent and thereafter could accept or decline participation in an option box. Next, participants answered some demographic questions. Participants were then instructed that they would have to read a text, and that some questions would be asked about it later in the survey. This text and the questions about its content were presented under the guise of a tool to assess their current attention state. Participants read the text and were subsequently asked to provide expectancy-value ratings of the 8 beliefs. The order of the presentation of the beliefs relating to disease and those unrelated to disease was randomized for each participant. Next, participants indicated their ratings on the direct measure of attitude and the control questions about the text's content. Lastly, participants were debriefed and were payed through Prolific Academic for their contribution.

\section{Statistical Analysis}

Theoretical model. It was expected that the regression coefficients of disease-related outcomes (EV1 to EV4) would increase in size (that is, deviation from zero) in the experimental group relative to control group. This change in regression coefficients deviation from zero is our operationalization of changes in belief importance. Additionally, diseaseunrelated beliefs (EV5 to EV8) on attitude were expected to decrease in size relative to the control group; indicating a decrease in importance of these beliefs (see Figure 1). However, given the direction of the product scales on EV4, EV5 and E6, we expected negative correlations with attitude, and thus expected that in line with our hypotheses the coefficient of EV4 would be more negative in the experimental group, but V5 and V6 would be less important and thus less negative in the experimental group. These effects were expected to occur regardless of any changes in the direct measure of attitude towards STI testing, because in principle changes in the relative importance of beliefs need not accompany overall changes in attitude, although (3) we expect that the direct measure of attitude towards STI testing is more positive in the pathogen-avoidance group as well.

Analysis. Data were analyzed with Mplus software (version 7.11). An expectancy-value model of attitudes towards STI testing was compared across groups in a multi-group latent variable model. Items designed as a direct measure of attitude were specified to load on a latent variable for attitude, and product scores for the expectancy-value ratings of the behavioral beliefs were used as predictors of the latent variable scores for attitude. That is, a variable was computed for all $\mathrm{E}{ }^{\mathrm{i}} \mathrm{V}$ I combinations and used as predictor of attitude towards STI testing. The model tested here thus does not use a composite score (i.e. $\Sigma\left(\mathrm{E}^{\mathrm{i} * \mathrm{~V}} \mathrm{i}\right)$ ) of expectancy-value rating - 
which assumes that each EV-product is an equally important predictor of an attitude, but allows for an inspection of how specific EV-products vary in their causal effect on attitude. This method of modelling behavioral beliefs as shaping an individual's attitude (also referred to as formative measurement), and a direct measure as reflective indicators of attitude allows assessing differences in belief importance across groups.

First, we ran a confirmatory factor analysis (CFA) and compared the factor structure of attitude on equivalence across groups. Such measurement invariance across groups is of importance because it assesses whether the attitude construct can be validly compared across groups, on both a mean difference as well as differences in how it is predicted by specific behavioral beliefs. Further, this model was then compared between control and experimental group to examine the effect of the pathogenavoidance manipulation on attitude, and most importantly, to assess whether the manipulation caused the hypothesized differences in the predictive strength of specific EV variables underlying an attitude (see Figure 1 for the conceptual model tested). This latter is assessed by a comparison of the regression coefficients across groups in a multi-group Multiple Indicator Multiple Causes (MIMIC) model (Joreskog \& Goldberger, 1975). All analyses used robust maximum-likelihood estimation (MLM), ChiSquare difference tests in model fit were calculated using a Satorra-Bentler scaling correction, which adjusts for deviations from normality.

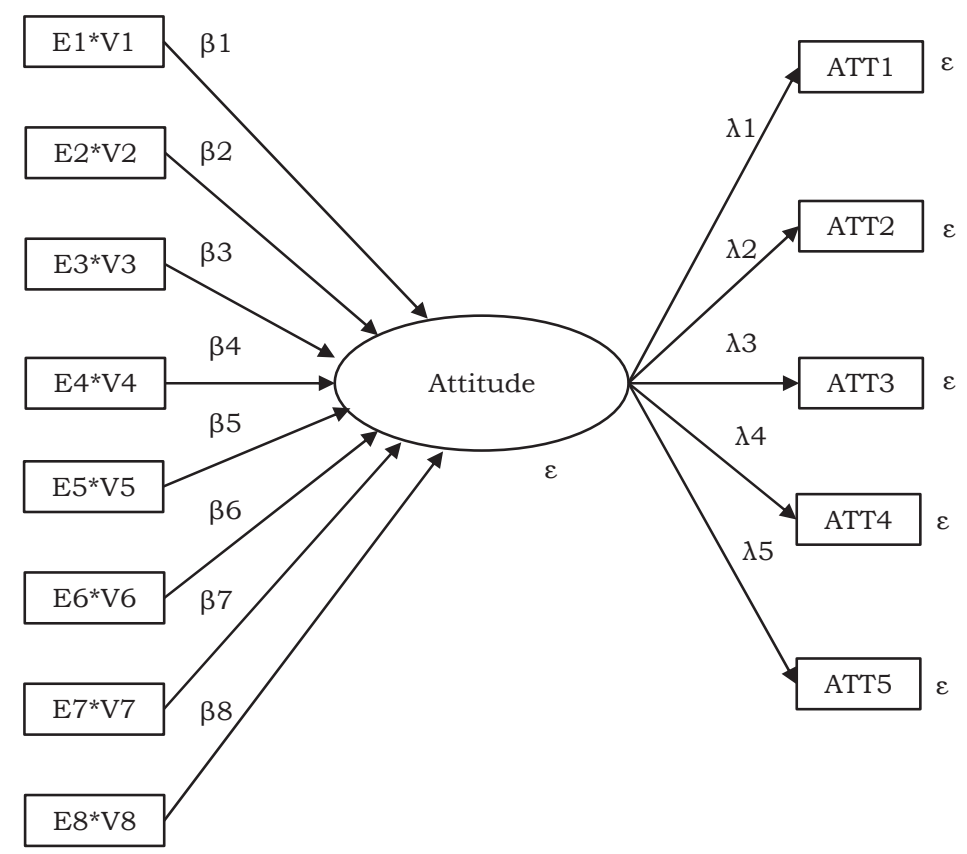

Figure 1. Conceptual model tested using a multi-group MIMIC model against hypothesized group differences. $\lambda=$ factor loading; $\beta=$ regression coefficient; $\varepsilon=$ error variance. ATT $=$ attitude 


\section{Results}

\section{Measurement invariance}

To assess whether our measurement of the attitude latent variable, using five indicators, was equivalent across groups, we first established metric invariance, that is, equivalence of the factor loadings across groups. The metric invariance model, where items were constrained to load equally on the attitude construct across groups, did not show worse fit to the data compared to a model where loadings were free to vary across groups $\left(\Delta x^{2}(4)\right.$ $=1.90, p=$. 754). This suggests that the items load equivalently on the attitude construct across groups. To assess whether the item intercepts were also equivalent across groups we tested a scalar invariance model, in which all factor loadings and intercepts were constrained equal across groups; this model also did not fit worse compared to our configural model where these were freely estimated $\left(\Delta x^{2}(8)=4.53, p=.807\right)$. This suggests that the item intercepts are also similar across groups, thus that similar responses can be expected on the items measuring attitude across conditions (see Table 2 for fit indices of the confirmatory factor analyses).

\section{Experimental manipulation and the direct measure of attitude}

To test whether the activation of a pathogen-avoidance motive influenced attitude scores across groups, we compared the latent mean values across groups. The difference between the latent mean scores of both groups was not significant (latent mean difference $=-0.148, z=-1.131$, $p=.258$ ). In addition to comparing latent mean differences across groups, we also conducted a $t$-test to evaluate a possible difference on the composite score of attitude between conditions. This analysis revealed a nonsignificant mean difference $(t[268]=1.318, p=.189 ; d=-0.16)$ between the control group $(M=6.57, S D=0.71)$ and the experimental group $(M=6.46$, $S D=0.76$ ). Thus, our experimental manipulation did not influence the direct measurement of attitude towards STI testing.

Table 2. Model fit statistics for the multi-group confirmatory factor and structural analyses.

\begin{tabular}{|c|c|c|c|c|c|c|c|c|}
\hline Measure & Model & $X^{2}$ & df & CFI & RMSEA & SRMR & $\Delta \mathrm{x}^{2}$ & $\Delta \mathrm{df}$ \\
\hline Attitudes & Configural (all free) & $4.495^{*}$ & 11 & 1.000 & 0.000 & 0.030 & --- & --- \\
\hline \multirow[t]{2}{*}{ (CFA) } & $\begin{array}{l}\text { Measurement } \\
\text { invariance }\end{array}$ & $6.412^{*}$ & 15 & 1.000 & 0.000 & 0.047 & $1.903 n s$ & 4 \\
\hline & $\begin{array}{l}\text { Scalar invariance } \\
\text { Equality of regression }\end{array}$ & $8.819^{*}$ & 19 & 1.000 & 0.000 & 0.050 & $4.562 n s$ & 8 \\
\hline Beliefs & Configural (all free) & $175.157^{*}$ & 84 & 0.924 & 0.090 & 0.044 & --- & --- \\
\hline \multirow[t]{4}{*}{ (SEM) } & Coefficients equal & 208.936* & 92 & 0.902 & 0.097 & 0.065 & $38.012 *$ & 8 \\
\hline & EV-4 free & 199.790* & 91 & 0.909 & 0.094 & 0.060 & $26.888^{*}$ & 7 \\
\hline & EV4/EV3 free & $189.369^{*}$ & 90 & 0.917 & 0.090 & 0.052 & $14,664 *$ & 6 \\
\hline & EV4/ EV3/EV7 free & $181.403^{*}$ & 89 & 0.923 & 0.088 & 0.047 & $5,268 \mathrm{~ns}$ & 5 \\
\hline
\end{tabular}

Note. The models testing attitudes involve invariance analysis of the attitude factor across groups. Models testing the measure beliefs involve a multi-group comparison of EV-beliefs as predictors of attitude across groups. $x^{2}$ - difference tests are Satorra-Bentler scaled; $d f=$ model degrees of freedom; CFI= comparative fit index; RMSEA= root-mean squared error of approximation; $\mathrm{SRMR}=$ standardized root mean squared residual. ${ }^{*}=$ significant $x^{2}$ at $\mathrm{p}<.05$. 


\section{Expectancy-value scores as a predictor of attitude}

In order to test whether activation of a pathogen-avoidance motive changes the relevance of specific behavioral beliefs underlying one's attitude rather than affects participants' overall attitude, we compared the predictive strength of the measured expectancy-value scores across groups (thus differences in $b$-values across groups). We compared a model in which these regression coefficients of the EV-predictors were constrained to be equal across groups to a model where all were allowed to freely vary. This resulted in significantly poorer fit for the constrained model $\left(\Delta x^{2}(8)=38.01, p\right.$ $<.0001)$ implying that there were differences in how attitude was predicted by specific EV scores.

We then inspected modification indices to examine which EV regression coefficients were different between the experimental and control group. After step-wise releasing constraints for EV3, EV4 and EV7 (see Table 1 for item content), the model fit was not worse anymore compared to a model where all coefficients were freely estimated $\left(\Delta x^{2}(5)=5.268, p=.384\right)$. Thus, the remaining coefficients can therefore be considered to not be statistically different between groups. Inspection of the coefficients shows that EV3 more strongly (see Table 1 for the items), and positively, predicted attitude in the experimental group compared to the control group, whereas EV4 and EV7 became weaker in this group (see Figure 2 for the results and a comparison of the models across groups), providing partial support for hypothesis 1 and 2 (see Table 2 for indices of the structural models).

To further probe how a pathogen-avoidance motivation influences expectancy-value ratings, we compared the control and experimental group on mean values of all eight EV-products. Conducted t-test revealed that there were no differences in mean EV values across groups on the eight EV variables (all p's > .05). Overall, these analyses indicate that 1) a pathogenavoidance motive did not influence attitude towards STI testing in our experimental group, and 2) the mean EV-scores on the behavioral beliefs were not different across groups, but 3) the manipulation did influence the importance of three specific behavioral beliefs underlying an individuals' attitude.

\section{Discussion}

In this study, we examined how priming a pathogen-avoidance motivation influences attitudes, and also the behavioral beliefs relevant to STI testing attitude. Overall, our results indicate that an essay prime intended to activate a pathogen-avoidance motivation did not cause changes in participants' attitudes towards STI testing. Neither were there any differences in mean EV-scores on the behavioral beliefs included in the current study. However, our analyses indicate that regardless of any changes in attitude or expectancy-value ratings, the pathogen-avoidance manipulation did affect the predictive strength of some expectancy-value 
components. We consider such changes in the predictive strength of specific behavior outcomes to reflect changes in the importance of these beliefs in forming an attitude given a certain context. This result suggests that belief importance might have value above and beyond expectancy-value ratings and attitude measures.

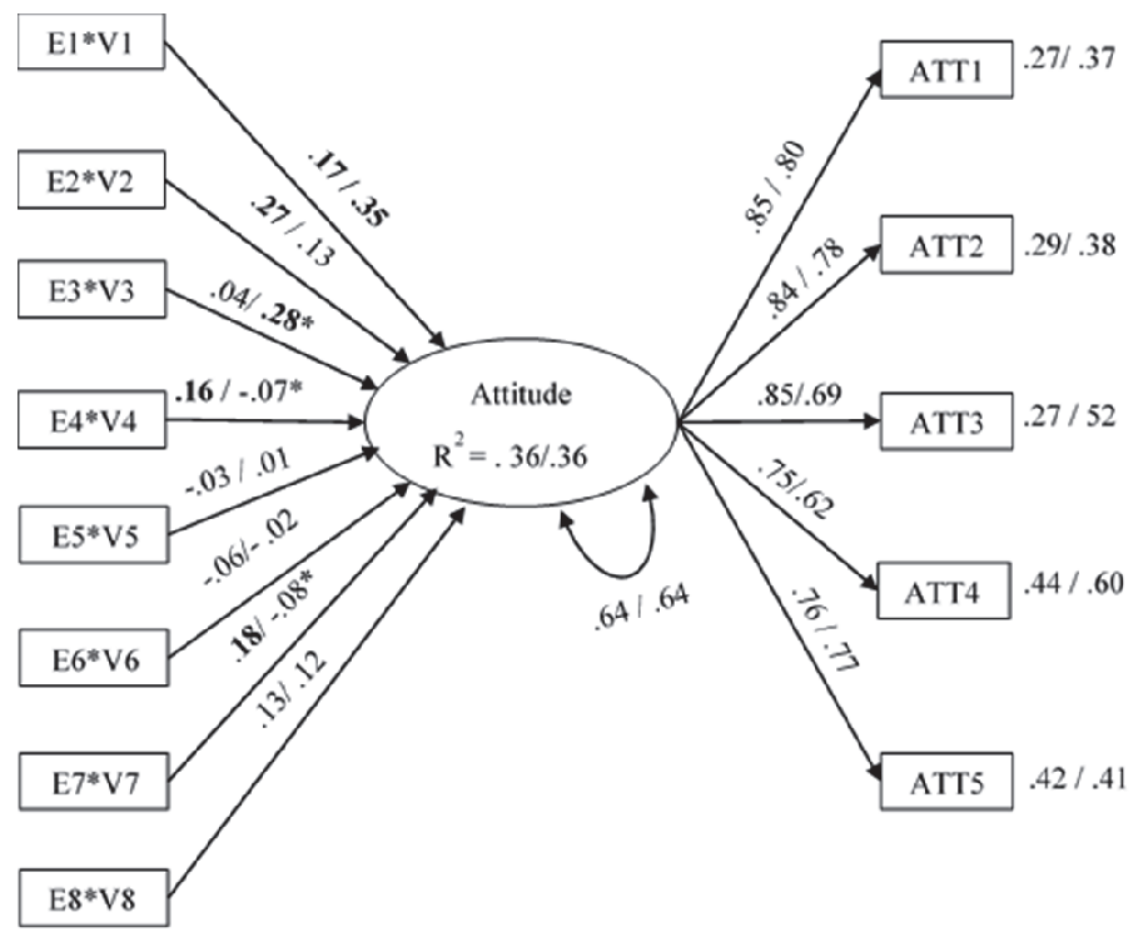

Figure 2. Results of the multi-group MIMIC comparison on STI-testing attitude and belief importance. The diagram represents two models. Coefficients left of the slash indicate estimates for the control group, whereas right of the slash denote the coefficients for the experimental group. Estimates are given for an unconstrained structural model where bcoefficients are freely estimated for both groups. Asterisks denote significant between-group differences in the coefficients. Boldfaced coefficients are significantly different from zero. Fit indices overall model: $\mathrm{x} 2=175.157, \mathrm{df}=84, \mathrm{p}<.001$ (CFI=0.924, RMSEA $=0.09$, SRMR $=$ $0.044)$.

Our results partially support our hypothesis that beliefs relating to disease-related aspects of STI-testing (EV1-EV4) would become stronger predictors of attitude as a result of the manipulation. That is, our results show that EV3 ("getting tested for sexually-transmitted diseases (STIs) would reduce my feelings of anxiety/fear of having contracted an STI") and EV4 ("getting tested for sexually-transmitted diseases (STIs) would cause stress and worry due to the possibility of testing positive") were more important beliefs underlying participants' attitude in the experimental condition than in the control condition. Although we expected a similar result for EV1 and EV2, inspection of the content of the items (see Table 1) does give some suggestion why especially EV3 and EV4 were affected by the 
manipulation, and not EV1 and EV2. As compared to EV1 and EV2, which ask about how important knowledge of infection and early treatment would be, both EV3 and EV4 asked about affective evaluations of STI testing behavior - i.e., preventing anxiety and the probability of experiencing stress and worry as a result of not testing. A possible explanation for why these beliefs became more important could be due to the fact that the pathogenavoidance manipulation explicitly targets affective - disgust related responses. Thus, affective (disease-related) aspects of behavioral outcomes associated with STI testing might have become more important because our manipulation especially influenced participants' affective state. In addition to these differences, the manipulation also decreased the relevance of nondisease related belief (EV7), inquiring about the importance of preventing transmission to other individuals - which is also in line with our theoretical model. Overall, the manipulation succeeded into changing the relevance of behavioral outcomes in the predicted direction, though this effect did not occur for all disease-related beliefs we included. The importance of these findings is underlined by the observation that (in addition to no changes in overall attitude) also the mean values of the EV-variables were not different across groups. Thus, across groups, participants rated these behavioral outcomes as equally desirable and likely. Overall, then, our results illustrate the notion that belief importance functions independently from expectancyvalue ratings and overall attitude towards a behavior.

Our finding that specific beliefs can become more important depending on a motivational state, regardless of any changes in a person's overall attitude towards a behavior, has several important implications for health psychology research. This study shows that measuring attitudes underlying health-protective behaviors, such as STI-testing, does not provide a full understanding of which considerations supposedly encompassed by this attitude are important to an individual. People can have similar attitudes based on different beliefs, but they can have the same overall attitude for different reasons - as we have illustrated here using a state motivation manipulation.

The fundamental motives framework may provide a basis for understanding which behavioral beliefs are important to people. Importantly, the fundamental motives framework emphasizes the fluidity of motivation, and situational and environmental factors are considered important to understand which motives might be cognitively prioritized (Kenrick, Griskevicius, et al., 2010; Kenrick, Neuberg, et al., 2010). Our approach here was to examine how fundamental motivations for behavior can influence attitude formation towards STI testing. Our Results underline the importance of not considering the beliefs and considerations individuals hold as static (cf. Harreveld et al., 2000), but as dynamic and influenced by state-level psychological processes. For health-promotion and intervention development, these findings highlight the need for continued effort into measuring not only the direct attitudinal, normative and control-related 
determinants of health behavior and their underlying beliefs, but also explicitly taking into account situational factors that might influence what people deem to be important.

\section{Limitations and suggestions for future research}

An important limitation of the current work is that the effect of our manipulation did not generalize to all belief-attitude relationships. Additionally, this study attempted to operationalize expectancy-value theory by using a novel MIMIC method with multi-group comparisons of regression coefficients. As specified by expectancy-value theory, products of likelihood and desirability rating of behavioral outcomes are assumed to formatively shape individual's attitude. Indeed, the model suggests that our set of modal salient beliefs accounted for thirty-six percent of the variance in attitude $\left(R^{2}\right.$ $=.36)$. However, the validity of expectancy-value product scores as predictors of attitudes is known to be dependent on the scaling of the component variables. There is disagreement, and long debate, over which method of item scaling and analysis leads to the best interpretable estimates of belief importance (French \& Hankins, 2003; Newton et al., 2012, 2014; O' Sullivan, McGee, \& Keegan, 2008). Given this so-called expectancy-value muddle, it may not be feasible to draw conclusions about the absolute relevance of the behavioral outcomes we examined in this study. However, we argue that many of these interpretational issues are avoided in the current study because we examined relative, between-group differences, and these tests of differences may be less susceptible to choice of item scaling and product computation.

Follow-up work is needed to examine whether the approach used to address questions of belief importance with our current methodology is indeed replicable and reliable. Additionally, given the unclear pattern of changes between groups due to the manipulation, replication of motivational priming effects on belief importance is also warranted. Nevertheless, this study has demonstrated that despite the absence changes in how people think about the outcomes of testing. Despite no changes in overall attitude towards STI testing, the importance of specific behavioral beliefs may depend on motivational states. This is a novel and important finding for research in health psychology, since it may suggest that measurement of people's specific beliefs underlying attitudes toward health behaviors does not give a clear picture on their importance. Thus, to understand how beliefs about the consequences of health behavior affect attitudes, intentions and actual behavior, it may be important - and necessary - to understand how context and state-specific the importance of beliefs is. 
Chapter 5: What is negative about testing for SEXUALLY-TRANSMITTED INFECTIONS?

This chapter is based on: Gruijters, S.L.K., Tybur, J.M., Massar, K., \& Ruiter, R.A.C. (under review). What is negative about testing for sexually-transmitted infections? 


\begin{abstract}
Shame and anticipated stigma are potential barriers to screening for sexually-transmitted diseases (STD). But are these expectations realistic? And - if so - what motivates stigmatization of STD-testers? Here, we test whether potential tradeoffs involved in managing the threats and affordances of sociality underlie such motives. In two studies, we examined whether individuals form more negative attitudes and attribute more shame to targets screened for STD. We also tested whether two threat-management motivations relate to these outcomes: avoidance of infectious and promiscuous individuals. Study $1 \quad(N=245)$ found positive associations between perceptions of a target's infectiousness and promiscuity and negative attitudes and attributed shame toward that target - but contrary to expectation, STD-testing decreased perceived infectiousness and promiscuity. Additionally, STD-testing by the target decreased participants' negative attitudes and attributed shame. Study $2(N=793)$ replicated these effects by experimentally manipulating information regarding target infectiousness and promiscuity. We conclude that STD-testing does not increase perceptions of infectiousness and promiscuity, but that these variables are independent sources of negative attitudes and attributed shame.
\end{abstract}




\section{Introduction}

Despite the many health benefits of early detection of sexuallytransmitted diseases (STD), testing rates are low even after potential exposure (e.g., Fortenberry, 1997; Malek, 2013). In addition to risk perceptions and lack of knowledge about test procedures (Richardson et al., 2010), low STD-testing rates partially stem from negative emotions and expectations - observers may perceive that going for a test connotes negative qualities of individuals. A large body of research has identified several such social barriers underlying limited uptake for STD (Barth, Cook, Downs, Switzer, \& Fischhoff, 2002; Cunningham, Kerrigan, Jennings, \& Ellen, 2009; Fortenberry, 1997; Lindberg, Lewis-Spruill, \& Crownover, 2006; Tilson et al., 2004). Two constructs are central in this literature. The first is stigma, defined as 'an attribute that results in widespread social disapproval' (Bos, Pryor, Reeder, \& Stutterheim, 2013, p. 2). The second is shame, 'a negative emotion elicited when a person experiences failure in relation to personal or social standards' (Fortenberry et al., 2002), which can follow from feeling stigmatized.

Evidence suggests that fear of stigma negatively relates to STDscreening behavior across various populations. For example, one study found that the expectation of STD-related stigma predicted lower Gonorrhea and HIV testing uptake (Fortenberry et al., 2002). Additional work suggests that adolescents who viewed STD-testing as stigmatizing were less likely to have gotten an STD test (Cunningham et al., 2009). Morris et al. (2014) observed that in a high-risk group of African American men, STD-related stigma predicted lower likelihood of screening, and these participants were also more reluctant to notify and deliver medication to partners. The relationship of experienced shame and STD-testing is less clear. Several studies indicate that shame does not retrospectively predict health care seeking (Cunningham et al., 2009; Fortenberry et al., 2002). However, focus groups with young urban adolescents (Lindberg et al., 2006) noted that fear of stigma, shame and embarrassment acted as significant barriers to testing. People further anticipate screening related negativity from others, such as being 'viewed as disgusting promiscuous risk-takers' (Balfe et al., 2010). Interviews by Barth et al. (2002) also honed in on stigma as a central theme when discussing barriers to STD-testing. College students in this sample reported anticipating being viewed as, for instance, loose, dirty, and irresponsible. Additional work also found that STD-infected people are perceived by others as less moral (Young, Nussbaum, \& Monin, 2007). While STD-related stigma and shame may form barriers to testing uptake, research has also shown that these feelings may promote health-protective behaviors by motivating increases in condom use (Sales et al., 2007).

This literature suggests that people anticipate negative responses from others and that such anticipations influence STD-related decision-making. Researchers aiming to develop tactics for addressing testing barriers can benefit from a better understanding of the origin of negative responses - or 
the anticipations of such responses - to STD testing. Here, we report studies that take a sociofunctional perspective on stigma and shame to test hypotheses on the reasons behind STD-related stigma (cf. Cottrell \& Neuberg, 2005; Cottrell, Neuberg, \& Li, 2007).

\section{A sociofunctional perspective on stigma}

From a sociofunctional perspective, human's sociality can be considered a 'double-edged sword'. That is to say, group life poses both affordances and threats to individuals' well-being. While cooperative alliances within groups are a defining feature of our species, and difficulties in maintaining these are posited to have driven human cognitive evolution (Dunbar, 1998; Dunbar, Shultz, Press, York, \& Nw, 2007; van Schaik, Isler, $\&$ Burkart, 2012), the benefits accrued by sociality also pose problems. Consider the following illustrative example of a pitfall of maintaining intense and various social ties: infectious disease. A significant number of viral and bacterial infections are contracted via social contagion. While some of these are relatively benign (e.g., Rhinovirus), many other infections have more serious health consequences. An STD such as Chlamydia can cause infertility when left untreated. Even pathogens perceived as relatively harmless - such as influenza - kill millions annually. Others - such as members of the Filoviridae taxon (one of which causes Ebola) and Human Immunodeficiency Virus (HIV) - have even more alarmingly high fatality rates. Natural selection can be expected to have both selected for 'cooperative' traits as well as traits enabling pathogen avoidance. However, the cost-benefit tradeoff (i.e. the described double -edged sword of sociality) between the two behaviors at least predicts constraints and an adaptive balance between pathogen avoidance and prosocial traits. As Kurzban and Leary (2001) put it: "for sociality to be functional, there must be "brakes" on sociality' (p.192).

Authors have posited that parts of human psychology evolved to navigate tradeoffs inherent to group living, such as the tradeoff between cooperation and pathogen-avoidance (Tooby \& Cosmides, 1990). One such model interpreted stigmatization as a response to potential threats to group efficiency and effectiveness (Neuberg et al., 2000). Phelan, Link, \& Dovidio (2008) argued that three specific functional aspects of stigmatization underlie the general function proposed by Neuberg and colleagues (2000); specifically, exploitation and domination of others, norm enforcement, and disease avoidance. Kurzban and Leary (2001) proposed stigmatization to encompass three functions, including 1) those pertaining to dyadic cooperation: avoiding non-valuable interaction partners, 2) coalitional exploitation: excluding individuals from gaining benefits of group membership, and 3) pathogen-avoidance: minimizing potential exposure to harmful pathogens.

A more recent model proposed by Oaten, Stevenson, and Case (2011) also connects stigmatization directly to a disease-avoidance system. These 
authors propose that an emotional component (disgust) directly reacts to perceptual disease cues (e.g., rashes), resulting in stigmatization. But the proposed model also involves cognitive components whereby learning disease labels (e.g., HIV, STD) are proposed to be an additional (higher-level) route to disease-avoidance responses and anticipatory anxiety. Thus, when individuals learn to associate a label with disease, the mere appreciation of the label content sufficiently triggers anxiety. This theoretical backdrop focusing on the (evolutionary) function of certain emotions and behaviors may also provide new insights into stigma-related social barriers to STD testing.

\section{Proximate mechanisms involved in regulating social threats}

Functional aspects of stigmatization (such as avoiding infection) supervene on the proximate cognitive and affective mechanisms causally involved in shaping behavior (cf. Scott-Phillips, Dickins, \& West, 2011). Emotions offer a good example of mechanisms that direct attention to relevant stimuli, including potential threats and affordances to well-being (see Neuberg, Kenrick, \& Schaller, 2011). One such emotion, disgust, has been implicated to regulate avoidance behavior across several domains. Although primarily seen as a pathogen-avoidance motivator (Curtis, 2011; Curtis et al., 2004, 2011), disgust motivates behaviors that neutralize other threats as well, including those pertaining to social coordination and undesirable sexual contact (for a review, Tybur, Lieberman, Kurzban, \& DeScioli, 2013). Disgust, framed as a multifaceted component of a threatmanagement system (cf. Neuberg et al., 2011), may therefore be a reasonable candidate for a potential underpinning of STD-related stigmatization. Social threats, such as unbeneficial cooperation partners and risk for disease-contraction, which evoke stigmatization (Kurzban \& Leary, 2001; Neuberg et al., 2000) directly link to the functional domains of disgust (see Tybur et al., 2013). This connection has been empirically tested in recent work.

For example, disgust sensitivity - referring to trait level reactivity towards disgust elicitors - predicted negativity towards obese people (Park et al., 2007), and has also been linked to negative attitudes towards the physically disabled (Park, 2003), foreigners (Navarrete \& Fessler, 2006), and homosexuals (Olatunji, 2008). At first blush, the connection between disgust sensitivity and the various attitudes described above may not seem straightforward. Many instances of disgust elicitors, including physical disabilities, obesity, and rashes, have little objective connection to potential infection threats. Why would disgust - understood as a pathogen-avoidance mechanism - relate to avoidance of such outcomes? Consider that functional behavior (e.g., avoiding pathogens) is achieved by proximate mechanisms that must separate signals (e.g., rash caused by microbes) from noise (false cue to infection). To illustrate, a rash on another person's arm may indicate the presence of an infectious agent or merely an allergic 
reaction. Theorists have argued that natural selection has likely biased perceptual decision-making to err towards the less costly side - heuristically, when in doubt whether something cues a threat, assume it to be a threat (Haselton et al., 2000; Haselton \& Buss, 2010; Neuberg \& Schaller, 2016; M. Schaller \& Park, 2011).

There is clear asymmetry in the fitness costs associated with making either Type-I errors (assuming that no pathogen is the cause of the rash, when in fact it is) and Type-II errors (assuming a pathogen is the cause, when in reality it is harmless). This notion has implications for understanding how people deal with the threats and affordances of sociality. It leads to the prediction that people respond emotionally to cues that connote non-valuable interaction partners (see Kurzban \& Leary, 2001). This prediction can roughly be couched in terms of a general functional heuristic: 'avoid deviants from the in-group norm', including physical deviance such as obesity and handicaps (Lieberman et al., 2012; Park, 2003; Park et al., 2007), or unfamiliarity due to outgroup membership or race (e.g., Cosmides, Tooby, \& Kurzban, 2003; Faulkner et al., 2004; Navarrete \& Fessler, 2006).

In the current paper, we suggest that getting tested for STD may also serve to flag 'social deviance' in a functionally specific way. If this is indeed the case, it is important to further understand what aspects of the STD testing 'flags' deviance, as to prevent anticipations of this to negatively affect testing behavior. To understand why people anticipate negative social responses of others when testing for STD, we aimed to further understand the kind of threats testing behavior might convey to others. We predicted that two perceived threats would be associated with STD-related negative perceptions. First, the responses may be associated with a pathogenavoidance motivation, in that STD-testers are perceived as 'infectious' - in line with the discussed functional model of stigma pertaining to pathogenavoidance. Second, the responses may be associated with a perceived threat imposed by the promiscuous behavior of others. It has been argued that it is in the interest of people those pursuing a relatively pursuing more monogamous reproductive strategy to condemn the promiscuous behavior of others (Kurzban, Dukes, \& Weeden, 2010; Tybur et al., 2015). Thus, this leads to the expectation that people may associate testing with promiscuous behavior, in turn causing stigmatization of STD-testers. We further predicted that given the importance of disgust sensitivity in explaining stigmatizing responses of individuals (Kurzban \& Leary, 2001; Lieberman et al., 2012; Oaten et al., 2011), this individual difference variable may play an explanatory role in understanding stigmatization related to STD-testing.

Finally, we take gender into consideration since the literature indicates gender differences in both norms pertaining to sexual behavior - the socalled 'sexual double standard', referring to gender conditional standards of sexual permissiveness (Crawford \& Popp, 2003; see also Kreager, Staff, Gauthier, Lefkowitz, \& Feinberg, 2016). Additionally, gender differences have 
been reported for disgust sensitivity (Lieberman et al., 2012; Tybur et al., 2009), and in the relationships between stigmatization and testing behavior (Cunningham et al., 2009). Therefore, we took both target and observer (participants) gender into account as a factor in the study designs. No specific expectations were set-up for the gender factors; we examined these factors exploratory. In two studies, we measured two manifestations of stigmatization: observers' negative attitudes and attributed shame towards a target (cf. Cunningham et al., 2009; Fortenberry et al., 2002).

\section{Study 1}

In Study 1 we explored the relevance of perceived infectiousness and promiscuity as predictors of negative attitudes and attributed shame in the context of STD testing. To this end, we designed vignettes that described a person either getting tested for STD or deciding not to get tested. Participants will be asked to indicate their attitude towards the described person. We do so to test 1) the influence of getting tested for STDs on negative attitudes and attributed shame, and 2) whether perceived promiscuity and infectiousness influence negative attitudes and attributed shame. We formulated the following hypotheses:

- Hypothesis 1. Getting tested for STD increases negative attitudes towards and shame attributed to the testee, thus we expect main effects of the testing condition on these outcomes.

- Hypothesis 2. Getting tested for STD increases other people's perceptions of a) infectiousness and b) promiscuity of the testee, thus we expect main effects of the testing condition on these outcomes.

- Hypothesis 3. Both perceived infectiousness and promiscuity are positively related to negative attitudes and attributed shame, thus we expect positive correlations between these measures.

- Hypothesis 4. Perceived infectiousness and promiscuity mediate any negative effect of getting tested on attributed shame and negative attitudes, thus we expect a positive mediation regression coefficient.

\section{Method}

\section{Participants and selection procedure}

Using a crowdsourcing platform (Prolific Academic; http:/prolific.ac.uk; see, Peer, Brandimarte, Samat, \& Acquisti, 2017) participants from the UK and the US were invited to participate. Given the nature of our manipulation, we limited our recruitment to students, native English speakers, aged 18-27 years. A total of 260 responses to the survey were recorded. After exclusion of participants that did not complete the survey $(n=4)$ and those who fell outside the pre-determined age criteria $(n=11)$, our final sample contained a total of $N=245$ participants, including 148 males $\left(M_{\text {age }}=21.8, S D=2.42\right)$ and 97 females $\left(M_{\text {age }}=21.9, S D=2.46\right)$. These participants were randomly assigned to a 2 (Testing: yes/no) x 2 (Gender vignette: male/female) between-subjects design. 


\section{Materials and Measures}

Vignettes. Four vignettes were designed to describe hypothetical students engaging in different sexual behaviors, and afterwards either going for an STD test or not. The vignettes for the four experimental conditions read as follows:

Thomas is a 21 year old student from Brighton. He recently moved from Brighton to London for his studies. Thomas is sexually active, but he is not planning to get tested for sexually-transmitted diseases (STD) at the health clinic.

Anna is a 21 year old student from Brighton. She recently moved from Brighton to London for her studies. Anna is sexually active, but she is not planning to get tested for sexually-transmitted diseases (STD) at the health clinic.

Thomas is a 21 year old student from Brighton. He recently moved from Brighton to London for his studies. Thomas is sexually active, and he has planned to get tested for sexually-transmitted diseases (STD) at the health clinic.

Anna is a 21 year old student from Brighton. She recently moved from Brighton to London for her studies. Anna is sexually active, and she has planned to get tested for sexually-transmitted diseases (STD) at the health clinic.

Perceived infectiousness. A measure with three items was designed to test the extent to which participants considered the individuals described in the vignettes as infectious: 1) 'How likely is it that this person is infected with a sexually-transmitted disease?', 2) 'How likely is it that this person will infect someone else with a sexually-transmitted disease in the future?', and 3) 'How likely is it that this person will be infected with a sexuallytransmitted disease in the future?'. These items were measured using 9point Likert scales ( 1 =extremely unlikely, $9=$ extremely likely).

Perceived promiscuity. To measure perceived promiscuity, we used the questions of the sociosexual orientation inventory pertaining to sexual behaviors (SOI; Penke \& Asendorpf, 2008) and rephrased them to reflect the behavior of the individuals in the vignettes. The items were: 1) 'Please estimate how many different partners this person has had sex with over the past 12 months.', 2) 'Please estimate with how many different partners this person had sexual intercourse on one and only one occasion.', and 3) 'Please estimate with how many different partners this person had sexual intercourse without having an interest in a long-term committed relationship'. These items were measured using a 9-point (non-linear scale), anchored on 1 (0 partners) to 9 (20+ partners).

Attributed shame. Based on research that utilized shame measures with items assessing anticipated shame or perceived shame (Cunningham et al., 2009; Fortenberry et al., 2002), we selected three items to assess attributed shame. These items were: 1) 'How ashamed should the described person feel about themself?', 2)'How embarrassed should the described 
person feel about themself?', 3) 'How disappointed should the described person feel about themself?'. Scales were 7-point Likert, ranging from 1 (Not at all) to 7 (Very Much).

Negative attitudes. Similar to attributed shame, we based our measure of negative attitudes on the stigma scales implemented by Cunningham et al. (2009) and Fortenberry et al. (2002). The scale included four items, 1) 'I would avoid the described person', 2) 'I would be uncomfortable around the described person.' 3) 'I would think the described person has bad morals', and 4) 'I would not want to be friends with the described person'.

Three Domain Disgust Scale. As a measure of our planned disgust sensitivity covariates, Study 1 included the Three Domain Disgust Scale (Tybur, Lieberman, \& Griskevicius, 2009; see for further validation Olatunji et al., 2012). This instrument assesses trait level disgust sensitivity across three domains - pathogen, sexual, and moral disgust-, each of which is assessed with seven items scored from 0 (not disgusting at all) to 6 (extremely disgusting). Pathogen disgust items inquire about disgust responses towards pathogen related concepts. For example, how disgusted participants would be after 'accidentally touching a person's bloody cut'. Sexual disgust measures a latent variable related to sexual aversions, for instance 'having an opposite sex stranger touching your thigh in an elevator'. Finally, moral disgust assesses disgust sensitivity towards moral transgressions, including responses to behaviors such as 'intentionally lying during a business transaction'.

\section{Procedure}

Approval for this study was obtained from the Ethics Review Committee of Psychology and Neuroscience at Maastricht University (code: ECP-04-092012). After enrolling in the study, participants were directed to the Qualtrics website (http://www.qualtrics.com), where they were asked to provide informed consent. Next, participants were randomly assigned to one of four conditions and were presented with a vignette description. They were subsequently asked to indicate their perceptions of the infectiousness and promiscuity of the individual in the vignette. Participants then completed the questions pertaining to attributed shame and negative attitudes. Lastly, participants completed the Three Domain Disgust Scale, and provided demographic information. After being debriefed about the study's hypotheses, they received compensation according to Prolific Academic payment standards.

\section{Statistical analyses}

Data processing was done using IBM SPSS (version 24) and $R$ software. Mplus software was used for confirmatory factor analysis. The measurement instruments used in the current study were assessed on unidimensionality and reliability, using confirmatory factor analysis (CFA) and the Omegatotal $\left(\omega^{t}\right)$ index with corresponding confidence intervals for reliability (Dunn, Baguley, \& Brunsden, 2014; Peters, 2014). Analysis of covariance was used 
to test the main study hypotheses relating to negative attitudes, attributed shame, infectiousness, and promiscuity (hypotheses 1-3). The models included only the planned disgust scale covariates with hypothesized effects on the outcome variables (cf. Gruijters, 2016). MANCOVA F-tests are based on Pillai's trace $(P T)$, and we report omega-squared $\left(\omega^{2}\right)$ effect size estimates (e.g., Okada, 2013). Hypotheses 4 and 5 were evaluated using multivariate mediation analyses, combined with estimates of indirect effects interpreted by constructing bootstrapped $(n=5000)$ confidence intervals for the regression estimates (e.g., Hayes \& Scharkow, 2013).

\section{Results}

\section{Descriptive statistics and scale validation}

Descriptive statistics of (and correlations between) the variables included in Study 1 are depicted in Table 1. Results of the CFA and reliability analyses indicated that the scales conformed to the hypothesized factor structure, and all measures had adequate reliability (see Table 2). The factor structure for pathogen disgust showed marginal fit to the data in the current sample (but see validation by Gruijters, Tybur, Ruiter, \& Massar, 2016; Olatunji et al., 2012; Tybur et al., 2009). Because attributed shame and negative attitudes were highly correlated $(r=.80)$ we opted to test the effects on these variables in MANOVA models to limit the number of significance tests. The uncorrelated promiscuity and infectiousness variables were examined in separate ANOVA models.

Table 1. Descriptives and correlations of Study 1.

\begin{tabular}{lllllllllll}
\hline Measure & Mean(SD) & 1. & 2. & 3. & 4. & 5. & 6. & 7. & 8. & 9 \\
\hline 1. Neg. Attitudes & $2.42(1.52)$ & --- & & & & & & & & \\
2. Att. Shame & $2.26(1.55)$ & $.80^{*}$ & --- & & & & & & & \\
3. Infectiousness & $5.22(1.59)$ & $.54^{*}$ & $.52^{*}$ & --- & & & & & & \\
4. Promiscuity & $4.30(1.51)$ & $.43^{*}$ & $.36^{*}$ & -.13 & --- & & & & & \\
5. Pathogen disgust & $4.64(.90)$ & .09 & .02 & .08 & .05 & --- & & & & \\
6. Sexual disgust & $3.27(1.28)$ & $.41^{*}$ & $.35^{*}$ & $.27^{*}$ & .01 & $.36^{*}$ & --- & & & \\
7. Moral disgust & $5.22(1.15)$ & -.07 & -.01 & .01 & -.12 & $.24^{*}$ & $.26^{*}$ & --- & & \\
8. Gender target & {$[.49-.51]$} & -.05 & -.01 & .03 & -.11 & -.04 & .01 & .06 & --- & \\
9. Gender observer & {$[.60-.40]$} & $-.26^{*}$ & $-.14^{*}$ & -.08 & $-.25^{*}$ & $.15^{*}$ & $.30^{*}$ & .04 & .002 & --- \\
\hline
\end{tabular}

Note. Gender is coded as [male-female].

\section{Multivariate analysis of covariance}

Results indicated that pathogen disgust and moral disgust did not correlate with negative attitudes, attributed shame, promiscuity and infectiousness, but sexual disgust did (see Table 1). Moral and pathogen disgust facets were therefore not included as covariates in the MANCOVA model. Before proceeding to test the MANCOVA model, we examined whether sexual disgust moderated any of the factors in the model; no such moderating effects were observed in the data (all $\left.\omega^{2}<.01\right)$. Therefore, we maintained sexual disgust as covariate. We further specified the MANCOVA 
on shame and negative attitudes according to our hypotheses: which included main effects for testing, target gender, observer gender, and sexual disgust as covariate.

\section{Attributed shame and negative attitudes}

There were non- significant interactions; testing condition with observer gender $\left(P T=.002, F(2,236)=.194, p=.823 ; \omega^{2}<.001\right)$, observer gender with target gender $\left(P T=.005, F(2,237)=.552, p=.557 ; \omega^{2}<.001\right)$, and testing with target gender $\left(P T=.02, F(2,238)=2.469, p=.087 ; \omega^{2}=.01\right)$. After removal of these non-significant interactions, the final model included three factors (observer gender, target gender, and testing condition), and sexual disgust as covariate. Testing condition had a moderately sized multivariate effect $\left(P T=.056, F(2,239)=7.103, p=.001 ; \omega^{2}=.05\right)$, indicating that negative attitudes and attributed shame decreased when the person in the vignette decided to get tested for STD. This analysis therefore provides evidence against Hypothesis 1, which predicted that getting tested for STD increases negative attitudes and attributed shame. Additionally, observer gender had a large multivariate effect $(P T=.205, F(2,239)=30.809, p<.001$; $\omega^{2}=.20$ ); female participants had less negative attitudes and attributed less shame to STD-testers than did male participants. The size of the main effect of target gender was near zero and non-significant $(P T=.007, F(2,239)=.901$, $\left.p=.408 ; \omega^{2}<.001\right)$. The sexual disgust covariate had a large effect on the outcome variables as well $(P T=.282, F(2,239)=46.894, p<.001$; see Table 2 for correlations).

Subsequent univariate tests indicated that all multivariate effects were visible on both outcome variables (all $p$ 's $<.05$ ), but the univariate effect of getting tested for STD on negative attitudes was substantially smaller $\left(F(1,240)=3.875, p=.05 ; \omega^{2}=.012\right)$ compared to its effect on attributed shame $\left(F(1,240)=13.022, p<.001 ; \omega^{2}=.047\right)$. Figure 1 provides violin plots of the observed main effects, unadjusted for covariates.

Table 2. Confirmatory factor - and reliability analysis (Study 1).

\begin{tabular}{lllllllll}
\hline Measure & $x^{2}$ & df & CFI & RMSEA & $90 \%$ CI & SRMR & $\omega^{\mathrm{t}}$ & $95 \%$ CI \\
\hline Infectiousness & 3.647 & 2 & 0.996 & 0.060 & {$[0.000 ; 0.156]$} & .026 & .88 & {$[0.85 ; 0.91]$} \\
Promiscuity & 21.305 & 2 & 0.901 & 0.198 & {$[0.128 ; 0.279]$} & .088 & .76 & {$[0.71 ; 0.81]$} \\
Neg. attitudes & 11.255 & 2 & 0.984 & 0.137 & {$[0.067 ; 0.220]$} & .017 & .90 & {$[0.88 ; 0.92]$} \\
Att. shame & 7.355 & 2 & 0.993 & 0.105 & {$[0.031 ; 0.190]$} & .012 & .95 & {$[0.94 ; 0.96]$} \\
Combined & 157.517 & 14 & 0.909 & 0.205 & {$[0.176 ; 0.234]$} & .053 & .94 & {$[0.93 ; 0.95]$} \\
PD & 51.761 & 14 & 0.821 & 0.105 & {$[0.075 ; 0.136]$} & .060 & .67 & {$[0.61 ; 0.73]$} \\
SD & 33.954 & 14 & 0.956 & 0.076 & {$[0.044 ; 0.109]$} & .041 & .81 & {$[0.77 ; 0.84]$} \\
MD & 64.540 & 14 & 0.924 & 0.043 & {$[0.092 ; 0.152]$} & .043 & .86 & {$[0.83 ; 0.89]$} \\
\hline
\end{tabular}

Note. $\omega^{\mathrm{t}}=$ omega-total reliability index. Abbreviations: $\mathrm{PD}=$ pathogen disgust, $\mathrm{SD}=$ Sexual disgust, $\mathrm{MD}=$ Moral disgust, $\mathrm{Att}=$ attributed, $\mathrm{df}=$ degrees of freedom, $\mathrm{CFI}=$ comparative fit index, RMSEA=root mean square error of approximation, $\mathrm{SRMR}=$ standardized root mean residual, $\mathrm{CI}=$ confidence interval for estimate. 


\section{Infectiousness and promiscuity}

We ran ANCOVA to test effects on the infectiousness and promiscuity scores (See Table 2). Like the previous MANOVA, the model included testing condition, observer gender, target gender, and sexual disgust as a covariate (and the exploratory two-way interactions). For infectiousness ratings, results indicated no presence of meaningful interactions (all $\omega^{2}<.01$ ). After removal of these interaction terms, the analysis indicated an effect of testing on infectiousness ratings, $F(2,227)=4.071, p=.045 ; \omega^{2}=.02$. This effect suggests that testing slightly decreases perceived infectiousness, providing evidence against Hypothesis $2 \mathrm{a}$, which predicted that perceptions of infectiousness would increase when people get tested for STD. Further, we observed gender differences on this outcome measure: male participants perceived higher infection probability than females $(F(2,227)=7.341, p$ $\left.=.045 ; \omega^{2}=.007\right)$. There was no effect of target gender on perceived infectiousness $\left(F(2,227)=.424, p=.667 ; \omega^{2}<.01\right)$. Finally, there was a large effect of sexual disgust on perceived infectiousness $(F(2,227)=22.866$, $\left.p<.001 ; \omega^{2}=.16\right)$, indicating that higher sexual disgust scores predicted higher infectiousness ratings.

We further analyzed the univariate effects on perceived promiscuity. First, there were no interactions between the factors (all $\left.\omega^{2}<.01\right)$. There was no main effect of the testing condition on promiscuity $(F(2,240)=.885, p$ $\left.=.348 ; \omega^{2}<.01\right)$, providing evidence against Hypothesis $2 \mathrm{~b}$. However, there was a large effect of observer gender $\left(F(2,240)=16.404, p<.001 ; \omega^{2}=.11\right)$, suggesting that females rated the targets on average as less promiscuous, and a small (non-significant) effect of target gender $(F(2,240)=3.167, p$ $\left.=.076 ; \omega^{2}=.02\right)$. Figure 2 gives means and distributions for the observed main effects of STD testing and observer gender.

\section{Mediation analysis}

To test whether the effects of testing and observer gender on perceived shame and negative attitudes towards people who get tested is explained by perceptions of infectiousness and/or promiscuity, we set up a multivariate regression model (with indirect effect estimates and controlling for sexual disgust). Results indicated that the hypothesized mediation model sufficiently fit the data, $x^{2}(1)=25.725, p<.001$ (CFI= 0.951, RMSEA $=0.318$, SRMR $=0.057)$. There was an indirect effect of the testing condition on negative attitudes, via perceived infectiousness $(b=-.043 ; 95 \% B C I=[-.083$; -.003]). Similarly, the testing effect on attributed shame was also mediated by perceived infectiousness $(b=-.045 ; 95 \% B C I=[-.087 ;-.003])$. The indirect effects from testing through promiscuity scores on negative attitudes $(b=.015 ; 95 \% B C I=[-.018 ; .043])$ and attributed shame $(b=.013$; $95 \% B C I=[-.016 ; .043])$ were both not significant.

Overall, these results suggest that getting tested for STD, through decreasing perceived infectiousness, relates to decreases in negative attitudes and attributed shame. There is less evidence for a mediation effect 
of promiscuity on the relationship between testing and the outcomes. These results support Hypothesis 3, which predicted that promiscuity and infectiousness are both related to negative attitudes and attributed shame. Hypothesis 4, which predicted mediation effects of infectiousness and promiscuity on the outcomes, was only partly confirmed by these analyses: only infectiousness mediated the STD testing effect on the outcomes but not in the expected positive direction. Finally, the effect of participant gender on negative attitudes was partially explained by both perceptions of infectiousness $(b=-.056 ; 95 \% B C I=[-.099 ;-.014])$ and promiscuity $(b$ $=-.070 ; 95 \% B C I=[-.122 ;-.028])$. Perceived infectiousness $(b=-.059 ; 95 \%$ $B C I=[-.105 ;-.013])$ and promiscuity $(b=-.061 ; 95 \% B C I=[-.099 ;-.023])$ also mediated the participant gender effect on the shame outcome variable.

\section{Discussion}

Study 1 examined the effects of STD testing on attributed shame and negative attitudes by manipulating descriptions of a target individual. We found that, contrary to expectations, getting tested for STDs decreases both attributed shame and negative attitudes towards the person getting tested. In line with our hypotheses, we found that perceptions of target infectiousness and promiscuity strongly predicted negative attitudes and attributed shame, such that higher perceived infectiousness and promiscuity were associated with increased negative attitudes and attributed shame. Finally, the effect of testing was mediated by infectiousness, suggesting that lowering this perception may be a potential causal mechanism by which testing decreases STD related stigma. No such evidence was found for perceptions of promiscuity. A limitation of Study 1 was that infectiousness and promiscuity were not manipulated. For this reason, it is possible that the correlation between these variables and negative attitudes and attributed shame reflects a general dislike or 'condemnation', rather than a response to promiscuity and infectiousness of the person. Study 2 was designed to resolve these issues. 
Chapter 5

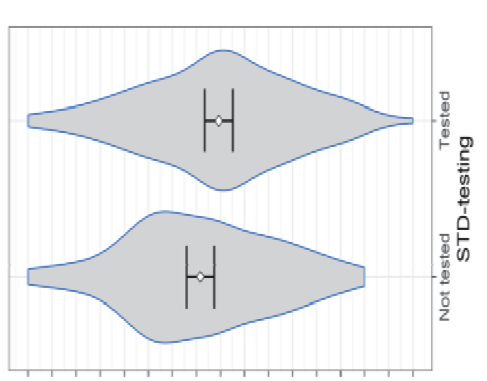

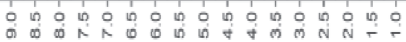
ssausno!̣วeju|

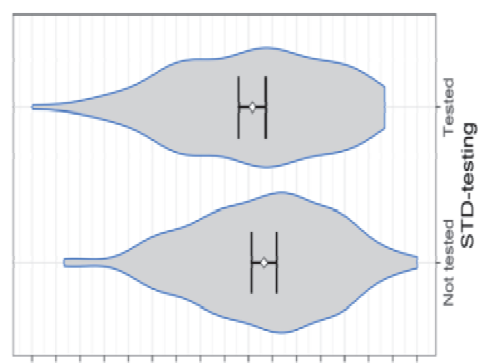

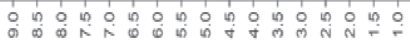

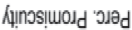
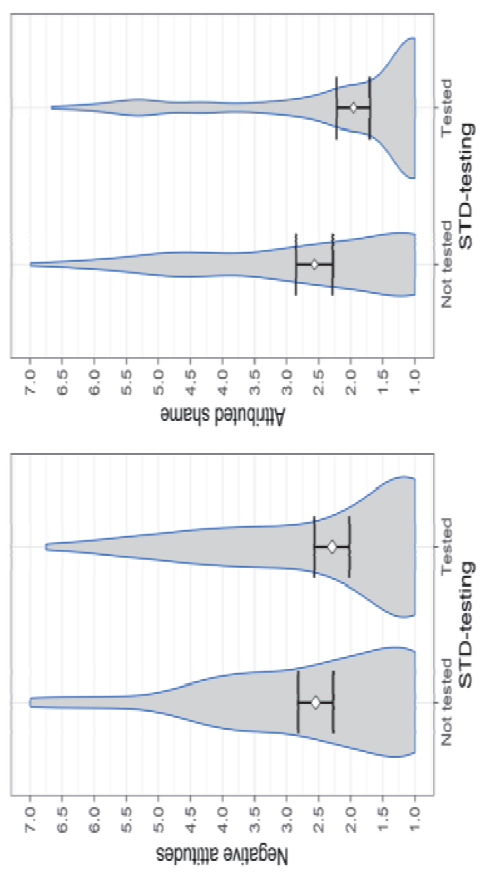

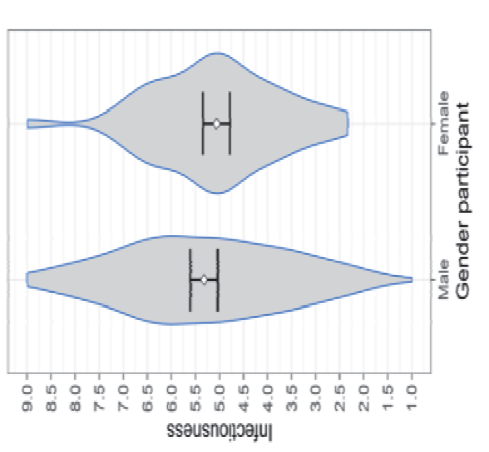

$\Xi$ 넝

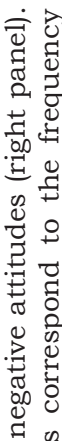

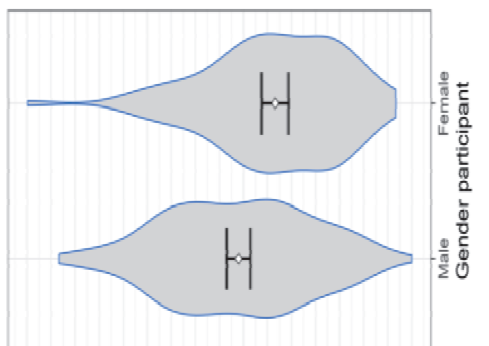

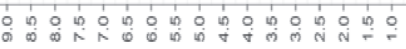

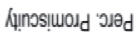
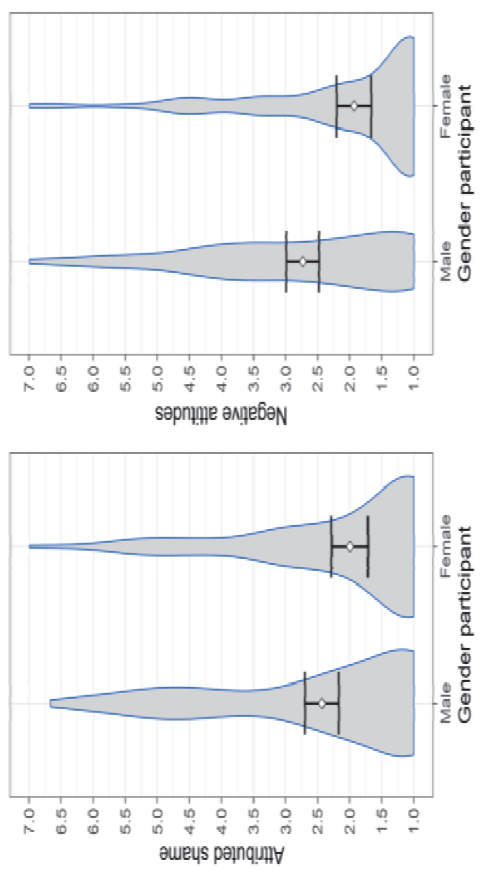

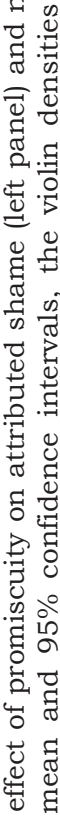

త్ర

兘

茴:

is on $\dot{\overline{7}}$

들. 总

寻总语

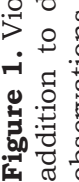




\section{Study 2}

In this study, vignettes were designed to manipulate not only testing status, but also sexual promiscuity and infection likelihood. As opposed to Study 1, the current design would allow causal conclusions about the relationship between perceptions of infectiousness and promiscuity and resulting negative attitudes and attributed shame. Thus, Study 2 provides a replication of the STD-testing effect on negative attitudes and attributed shame, and a experimental follow-up of the relationships between promiscuity and infectiousness on negative attitudes and attributed shame. Furthering on the results of Study 1, we hypothesized the following:

Hypothesis 1: Getting tested for STD decreases negative attitudes and attributed shame of others.

Hypothesis 2: Higher promiscuity levels (number of sex partners) leads to stronger negative attitudes and more attributed shame

Hypothesis 3: Higher infectiousness likelihood (unsafe sex with an infected partner) increases negative attitudes and more attributed shame.

\section{Methods}

\section{Participants and selection procedure}

Similar to Study 1, participants from the UK were invited through Prolific Academic. Inclusion criteria were also being a native English speaker, aged between 18-27. A total of 814 complete responses to the survey were recorded. After exclusion of participants that did not complete the survey $(n=11)$ and those who did not fall within the pre-determined age criteria $(n=10)$, our final analytic sample contained a total of $N=793$ participants $\left(M_{\text {age }}=21.4, S D=2.42\right)$, including 441 males $\left(M_{\text {age }}=21.3, S D=2.48\right), 350$ females $\left(M_{\text {age }}=21.6, S D=2.33\right)$, and two participants who did not report their sex. Participants were randomly assigned to a 2 (testing: no/yes) $\mathrm{x} 2$ (infectiousness: low/high) x 2 (promiscuity: low/high) x 2 (target gender: female/male) between-subjects design.

\section{Materials and Measures}

Vignettes. Furthering on Study 1, we designed vignettes to tap into the relevance of infectiousness, promiscuity and getting tested for STD on stigma related outcomes. The manipulations were tested factorial in a 2 (infectiousness: low/high) x 2 (promiscuity: low/high) x 2 (testing: yes/no) between-subjects design. The vignettes described a person (either male or female) having had a certain number of sex partners. This number was either one partner in the last six months (low promiscuity) or five partners in the last month (high promiscuity). Infectiousness was also manipulated in the vignettes, by describing that the target person either did not always have safe sex and had a previous partner who tested positive for infections (high infectiousness), or by describing the target person as someone who always 
had safe sex and nothing was disclosed about potential infection status by a partner (low infectiousness). This person, after seeing a testing brochure, did or did not decide to get tested for STI. An example vignette (for high infectiousness, high promiscuity, and a female target) is as follows:

Anna is a 23 year old student from Brighton. She recently moved from Brighton to London for her studies. In the last month, Anna has had 5 sexual partners. Anna and her partners did not always use condoms. Just after Anna moved to London, one of these 5 partners informed Anna that he recently tested positive for a sexually-transmitted disease. When Anna walked past a testing brochure, she considered to get tested for sexuallytransmitted diseases, and she decided to go to the sexual health clinic to be tested.

Box 2 contains the full set of vignettes used in this study.

Attributed shame. The measurement of attributed shame was similar to Study 1 , and consisted of three items using 7 -point Likert scales ( $1=$ not at all, $7=$ very much). The items were: 1) 'How ashamed should [Anna] feel about herself?', 2) 'How embarrassed should [Anna] feel about herself?', and 3) 'How disappointed should [Anna] feel about herself?'. In the male versions of the scenario, the name was replaced by 'Thomas' and appropriate changes to the personal pronouns were made.

Negative attitudes. The same four items as in Study 1 measured negative attitudes similarly on 7-point Likert scales, the items included 1) 'I would avoid [Anna]', 2) 'I would be uncomfortable around [Anna]', 3) 'I would think [Anna] has bad morals', and 4) 'I would not want to be friends with [Anna]'.

Disgust sensitivity. We again used the Three Domain Disgust Scale to measure disgust sensitivity, as described in Study 1 materials section. Given the results of this measure in Study 1, here, we opted to only measure the sexual and pathogen disgust facets of this scale. 
Box 2. Vignettes used in Study 2.

Testing factor $(\mathrm{NT}=$ not tested $/ \mathrm{T}=$ tested $)$.

Infectiousness factor ( $\mathrm{HI}=$ high infectiousness, $\mathrm{LI}=$ low infectiousness).

Promiscuity factor ( $\mathrm{HP}=$ high promiscuity, $\mathrm{LP}=$ low promiscuity).

Gender factor ( $\mathrm{F}=$ female, $\mathrm{M}=$ male).

NT-HIHP-F Anna is a 23 year old student from Brighton. She recently moved from Brighton to London for her studies. In the last month, Anna has had 5 sexual partners. During these sexual encounters, Anna and her partners did not always use condoms. Some time ago, one of these 5 partners informed Anna that he recently tested positive for a sexuallytransmitted disease. Anna thought about getting tested for sexuallytransmitted diseases when she moved to London, but she decided not to.

$N T-L I H P-F$ Anna is a 23 year old student from Brighton. She recently moved from Brighton to London for his studies. In the last month, Anna has had 5 sexual partners. During these sexual encounters, Anna and her partners always used condoms. Anna thought about getting tested for sexually-transmitted diseases when she moved to London, but she decided not to.

NT-HILP-F Anna is a 23 year old student from Brighton. She recently moved from Brighton to London for her studies. In the last 6 months, Anna has had 1 sexual partner. Anna and her partner did not always use condoms. Some time ago, this partner informed Anna that he recently tested positive for a sexually-transmitted disease. Anna thought about getting tested for sexually-transmitted diseases when she moved to London, but she decided not to.

NT-LILP-F Anna is a 23 year old student from Brighton. She recently moved from Brighton to London for her studies. In the last 6 months, Anna has had 1 sexual partner. Anna and her partner always used condoms. Anna thought about getting tested for sexually-transmitted diseases when she moved to London, but she decided not to.

T-HIHP-F Anna is a 23 year old student from Brighton. She recently moved from Brighton to London for her studies. In the last month, Anna has had 5 sexual partners. Anna and her partners did not always use condoms. Some time ago, one of these 5 partners informed Anna that he recently tested positive for a sexually-transmitted disease. Anna thought about getting tested for sexually-transmitted diseases when she moved to London, and she decided to go to the sexual health clinic to be tested.

$T-L I H P-F$ Anna is a 23 year old student from Brighton. She recently moved from Brighton to London for her studies. In the last month, Anna has had 5 sexual partners. Anna and her partners always used condoms during these sexual encounters. Anna thought about getting tested for sexuallytransmitted diseases when she moved to London, and she decided to go to the sexual health clinic to be tested. 


\section{Box 2 (continued)}

T-HILP-F Anna is a 23 year old student from Brighton. She recently moved from Brighton to London for her studies. In the last 6 months, Anna has had 1 sexual partner. Anna and her partner did not always use condoms. Some time ago, this partner informed Anna that he recently tested positive for a sexually-transmitted disease. Anna thought about getting tested for sexually-transmitted diseases when she moved to London, and she decided to go to the sexual health clinic to be tested.

$T-L I L P-F$ Anna is a 23 year old student from Brighton. She recently moved from Brighton to London for his studies. In the last 6 months, Anna has had 1 sexual partner. Anna and her partner always used condoms during their sexual encounters. Anna thought about getting tested for sexually-transmitted diseases when she moved to London, and she decided to go to the sexual health clinic to be tested.

Note. This box only displays the female versions. Male vignettes described a student named Thomas.

\section{Procedure}

Participants were asked to carefully read a description of a person and were shown 1 out of 16 vignettes. After having the read the vignette, they were asked to complete the items measuring attributed shame and negative attitudes. Next, they completed the sexual disgust and pathogen disgust questions and ended the survey with providing their demographics.

\section{Results}

\section{Descriptive statistics and scale validation}

The descriptive statistics of (and correlations between) the variables included in Study 2 are depicted in Table 3. Results of the CFA and reliability analyses indicated that all measures had adequate reliability and that the scales conformed to the hypothesized factor structure (See Table 4).

Table 3. Descriptive statistics and correlations between the study variables (Study 2).

\begin{tabular}{llllllll}
\hline Measure & $\mathrm{M}(S D)$ & 1 & 2 & 3 & 4 & 5 & 6 \\
\hline 1. Neg. Attitude & $2.39(1.54)$ & $(-)$ & & & & & \\
2. Att. Shame & $2.51(1.75)$ & $.79^{*}$ & $(-)$ & & & & \\
3. Pathogen disgust & $4.69(1.02)$ & $.10^{*}$ & $.08^{*}$ & $(-)$ & & & \\
4. Sexual disgust & $3.29(1.26)$ & $.25^{*}$ & $.23^{*}$ & $.31^{*}$ & $(-)$ & & \\
5. Gender target & {$[0.49-0.51]$} & $-.13^{*}$ & $-.12^{*}$ & .01 & .02 & $(-)$ & \\
6. Gender observer & {$[0.56-0.44]$} & $-.16^{*}$ & $-.13^{*}$ & $.09^{*}$ & $.28^{*}$ & .05 & $(-)$ \\
\hline
\end{tabular}

Note. Gender is coded as $[0=$ male; $1=$ female $]$ 


\section{Multivariate analysis of covariance}

We specified the MANCOVA according to our hypotheses, which included main effects for promiscuity, infectiousness, testing, observer gender, target gender, and sexual and pathogen disgust as covariate. Similar to Study 1, we examined only multivariate effects on the dependent variables (negative attitudes and attributed shame), given a high correlation between these measures $(r=.80)$. Results indicate that pathogen-disgust did not moderate any of the factors (all interaction term $p$-values $>.05$ ), and after removal of the interactions was not a significant covariate in the model ( $P T$ $\left.=.005, F(2,781)=1.799, p=.166 ; \omega^{2}<.01\right)$. However, the relationship of sexual disgust with the outcome variables was moderated by target gender $\left(P T=.012, F(2,782)=4.620, p=.010 ; \omega^{2}=.010\right)$. Therefore, we maintained an interaction term for sexual disgust and the target gender factor in our model. Sexual disgust was further a significant covariate with a large effect on the outcomes $\left(P T=.123, F(2,782)=54.619, p<.001 ; \omega^{2}=.12\right)$.

Table 4. Confirmatory factor - and reliability analysis (Study 2).

\begin{tabular}{|c|c|c|c|c|c|c|c|c|}
\hline Role & Measure & df & $\mathrm{CFI}$ & RMSEA & $90 \% \mathrm{CI}$ & SRMR & $\omega^{\mathrm{t}}$ & $95 \%$ CI \\
\hline \multirow[t]{3}{*}{ Dv. } & Neg. Attitude & $33.961 * 2$ & 0.985 & 0.142 & {$[0.102 ; 0.186]$} & 0.016 & 0.91 & {$[0.90 ; 0.92]$} \\
\hline & Att. Shame & $10.181 * 2$ & 0.996 & 0.072 & {$[0.033 ; 0.118]$} & 0.008 & 0.95 & [0.94;0.95] \\
\hline & Combined & $567.639 * 14$ & 0.897 & 0.223 & [0.208; 0.239$]$ & 0.054 & 0.95 & {$[0.94 ; 0.95]$} \\
\hline \multirow[t]{2}{*}{ Cov. } & PD & $143.720 * 14$ & 0.882 & 0.108 & {$[0.092 ; 0.124]$} & 0.052 & 0.75 & {$[0.72 ; 0.78]$} \\
\hline & SD & $161.502 * 14$ & 0.908 & 0.115 & {$[0.100 ; 0.132]$} & 0.052 & 0.81 & {$[0.78 ; 0.83]$} \\
\hline
\end{tabular}

Note. $\omega^{\mathrm{t}}=$ omega-total reliability index. Abbreviations: Dv. $=$ dependent variable, Cov. $=$ covariate, $\mathrm{PD}=$ pathogen disgust, $\mathrm{SD}=$ Sexual disgust, $\mathrm{df}=$ degrees of freedom, $\mathrm{CFI}=$ comparative fit index, RMSEA=root mean square error of approximation, $\mathrm{SRMR}=$ standardized root mean residual, $\mathrm{CI}=$ confidence interval for estimate.

* significant $x^{2}$ at $\mathrm{p}<.05$.

Main effect analysis. Results of the multivariate main effect analysis showed a moderately sized main effect of the testing manipulation $(P T=.068$, $\left.F(2,782)=28.737, p<.001 ; \omega^{2}=.07\right)$, a large effect of the infectiousness manipulation $\left(P T=.240, F(2,782)=123.393, p<.001 ; \omega^{2}=.24\right)$, and a moderate effect size for the promiscuity manipulation $(P T=.063$, $\left.F(2,782)=26.090, p<.001 ; \omega^{2}=.06\right)$. These effects suggest that both higher levels promiscuity and infectiousness increased negative attitudes and attributed shame, whereas getting tested for STD decreased scores on these outcomes. Overall, these main effects provide support for Hypothesis 1-3, supporting the findings of Study 1 (see Figure 2). Main effect analysis further indicated that observed gender had a significant multivariate effect $\left(P T=.071, F(2,782)=29.873, p=>.001 ; \omega^{2}=.07\right)$, suggesting that male participants were on average more negative about the vignette descriptions (in line with Study 1).

Interaction analysis. We next analyzed the interaction between sexual disgust and target gender. Simple slopes were computed on -1SD, the mean, and $+1 \mathrm{SD}$ of sexual disgust, and examined in the MANOVA model. The 
multivariate effect of target gender was near zero given low sexual disgust $\left(P T=.003, F(2,782)=1.000, p=.368 ; \omega^{2}<.01\right)$, small when estimated at mean sexual disgust $\left(P T=.027, F(2,782)=10.972, p<.001 ; \omega^{2}=.02\right)$, and larger given high sexual disgust $\left(P T=.036, F(2,782)=14.544, p<.001 ; \omega^{2}\right.$ $=.03$ ). These effects suggest that males and females were perceived equally negatively when sexual disgust is low, but that especially male vignette descriptions evoked negative attitudes and shame for participants scoring high on sexual disgust.

\section{Discussion}

Study 2 provides a replication and experimental follow-up on the relationships between testing for STD, promiscuity, and infectiousness on stigma related outcomes. Replicating and extending Study 1, we found that infectiousness and promiscuity causally relate to negative attitudes and attributed shame, such that higher levels of infectious and promiscuity causes negativity by observers (see Figure 2). Additionally, we found that similar to the findings in Study 1 getting tested for STD decreases negative attitudes and attributed shame. Moreover, this latter effect observed in Study 2 substantiates the causal directions tested in the cross-sectional mediation model of Study 1. That is, from both the combined results of Study 1 and 2 we suggest that testing for STD causally relates to (less) perceived infectiousness, and further that both infectiousness and promiscuity causally relate to (less) negative attitudes and attributed shame. 

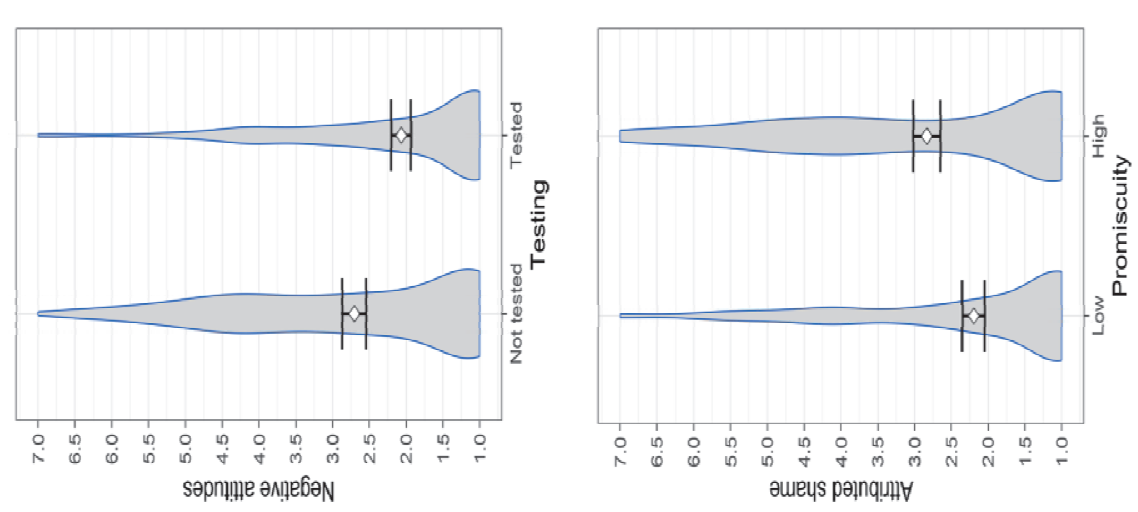

용

sәрпџџе әмпџебә
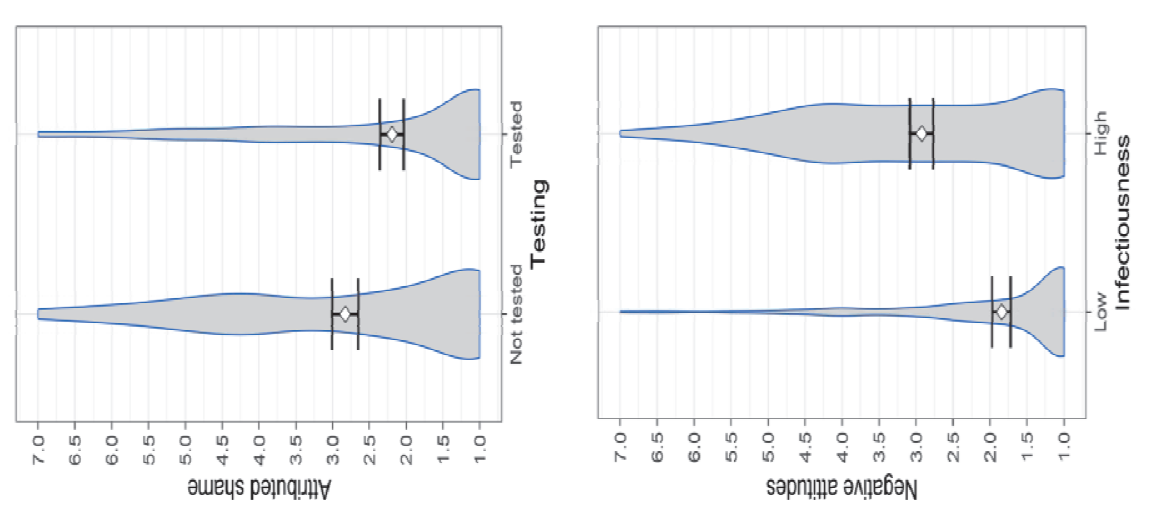

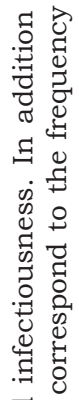

ชี ฮี

密

过

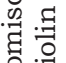

는

ดิ

要
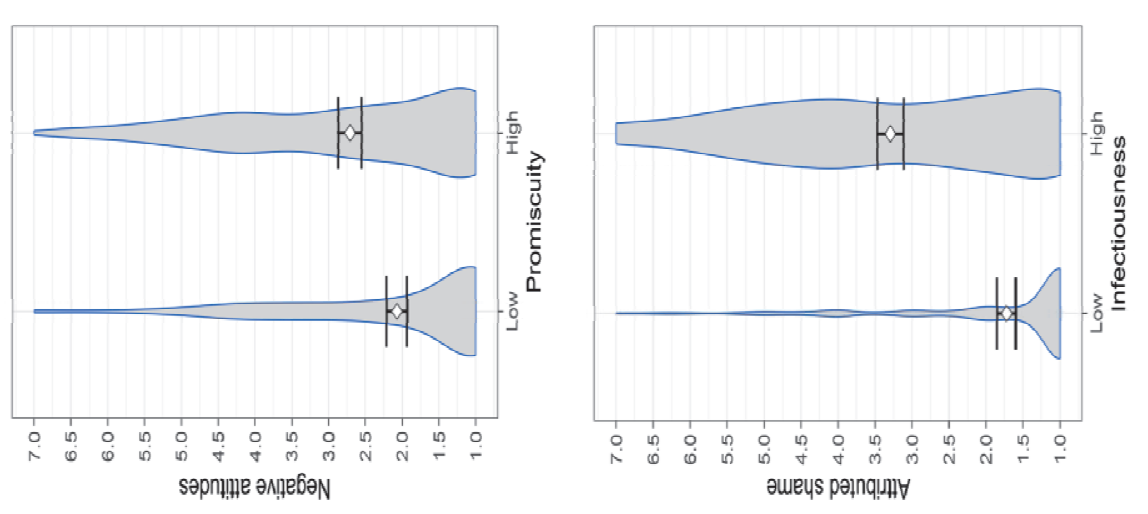

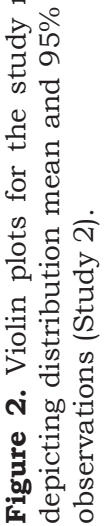




\section{General discussion}

Across two studies, we examined the roots of STD-related stigma by focusing on the information that testing may convey to others. Guided by a functional analysis of stigma, we proposed that two different motivations may underlie negativity towards those who get tested: stigmatization related to the perceived infectiousness, and stigmatization related to perceived promiscuity. Results showed clearly that both infectiousness and promiscuity positively predicted negative attitudes and attributed shame. However, and contrary to our predictions, getting tested for STD did not increase participants' negativity towards the target. Instead, testing for STD decreased perceptions infectiousness leading to more positive attitudes and less attributed shame. These findings suggest that negativity is not directed at STD testing behavior but rather at what going for a test conveys about the sexual behavior of individuals and the possible negative consequences of this behavior (e.g., infectiousness).

The results from Studies 1 and 2 indicated that perceptions of infectiousness are a strong predictor of negative attitudes toward that person, and it also strongly predicted attributed shame. These findings suggest that infection threat is important to understand negativity towards individuals, and is line with earlier theorizing on the functions of stigmatization. Social psychological models have a difficult time explaining why infectiousness would relate to stigmatization (Phelan et al., 2008), but these associations may be elucidated by considering functional models of stigma (e.g., Kurzban $\&$ Leary, 2001; Neuberg et al., 2000). These models predict that people social avoidance - and stigmatization- is an adaptive response to pathogen threats imposed by others. Although we did not find evidence that getting tested for STD serves as a similar (informational) cue, perceived infectiousness of the targets did predict negativity towards the target person. Thus, we suggest that this finding is in line with predictions from the Kurzban and Leary (2001) model, which suggests pathogen-avoidance to be one functional aspect of stigma. Therefore, we suggest that the observed effects of infectiousness (perceived in Study 1 and manipulated in Study 2) on negative attitudes and attributed shame to be manifestations of stigmatization that is functionally rooted in pathogen-avoidance motivation.

A second functional reason for negativity towards people who get tested for STD we proposed to be perceived promiscuity. While perceived promiscuity of the targets (like perceived infectiousness) did not increase as a result of getting tested for STD, perceptions of promiscuity did strongly predict negative attitudes and attributed shame (Study 1). This association was strong, also when promiscuity was manipulated (Study 2). The approach taken to understand stigmatization centers around functional processes to avoid interpersonal threats, and we suggest that the relationship between promiscuity and negative attitudes may indicate that promiscuous behavior is perceived as a threat by people favoring monogamous relationships, and thus negatively appraised (Kurzban et al., 
2010; Tybur et al., 2015). This finding is in line with work showing that promiscuous people are perceived as potentially sexually unfaithful (Bailey, Kirk, Zhu, Dunne, \& Martin, 2000), and as less desirable partners or close friends (Bleske \& Schackelford, 2001). While a large body of evidence suggests that negativity towards promiscuity may be qualified by a sexual 'double standard' (e.g., Sagebin Bordini \& Sperb, 2013; Zaikman, Marks, Young, \& Zeiber, 2016), we did not find such moderating effects of target gender in our sample and on the current measures.

Both the reported studies included individual difference measures of disgust sensitivity. Study 1 and 2 found that only sexual disgust sensitivity was related to perceived infectiousness, negative attitudes, and attributed shame. Individuals high in sexual disgust tend to prefer long-term, monogamous relationships, and they also tend to condemn behaviors and policies perceived as promoting (or, at least, allowing) relatively more promiscuous sexuality (Kurzban et al., 2010; Tybur et al., 2015). The relationship between trait sexual disgust and negative ratings of targets might reflect condemnation of non-monogamous sexual behavior by those who favor more monogamous relationships. Overall, these findings suggest that participants' interpersonal evaluations are related to sexual disgust sensitivity.

\section{Limitations and future directions}

The reported studies have limitations. First, the measures used to operationalize manifestations of stigmatization may be susceptible to social desirable responding by participants. Further, the highly skewed distributions of negative attitudes and attributed shame created a floor effect in the data, which may have attenuated statistical power. We found no evidence that getting tested for STD is related to stigma related outcomes, here operationalized with measures of negative attitudes and attributed shame. Instead, the data suggest that testing leads to more positive evaluations of these vignettes. Before concluding that testing for STD is indeed positively perceived and evaluated by others, we suggest that followup work is needed that uses less explicit methods to measure such evaluations. In addition to conducting several confirmatory tests of hypotheses, the reported studies also include several exploratory analyses. Both the unexpected direction of effects for our hypothesis tests and the exploratory nature of some analyses warrant replication efforts before inferences can be made with more certainty. However, the data reported here suggest that negativity towards STD-testers may be mediated by perceptions of infectiousness, and that testing for STD decreases overall negativity towards testers. Given a large literature proposing that anticipated negativity is a major barrier to testing, we suggest that this study may have important implications for health promotion research. Getting the message across that the fear of being labeled as promiscuous or infectiousness as a result of STD testing is not realistic, but rather perceived as positive, may be one way of decreasing this barrier to STD testing. 

Chapter 6: General discussion 


\section{Introduction}

The aim of this dissertation was to explore how an evolutionary perspective can help understand health behavior. Across four chapters, I theoretically and empirically explored what evolutionary perspectives can offer health psychological research, and tested specific predictions derived from this framework.

Chapter 2 aimed to first clarify what an evolutionary perspective on health behaviors holds, how it differs from standard psychological theory, and how it can potentially illuminate questions about health behaviors. Chapter 3 reported two cross-sectional studies that examined the hypothesis that a domain-specific motivation to avoid pathogens may provide novel insights in understanding health-protective behaviors, operationalized with measures of participants' attitudes and intentions to screen for infectious and non-infectious disease. Chapter 4 reported an experimental study that used an essay - textual - prime, designed to evoke a pathogen-disgust motivational state, to examine whether activation of such a motive would increase positive attitudes towards testing for sexuallytransmitted disease. This study also examined whether a potential effect would alter the relevance or importance of specific beliefs people have about the consequences of STD screening. Chapter 5 reported two studies that examined the flip-side of pathogen-avoidance motivation: rather than examining potentially reinforcing effects of pathogen avoidance on screening motivations, this study tested whether pathogen-avoidance motivation may be involved in generating barriers to testing for STI.

This chapter will discuss the findings reported in this dissertation, their implications and applications, the limitations of our studies, and the prospects for further investigations.

\section{Summary of main findings}

In Chapter 2, we reviewed the literature and examined the theoretical foundations of evolutionary approaches in psychology. We discussed the critical distinction between ultimate and proximate explanations for behavior, and suggested that whereas standard health behavior theory (HBT) considers proximate explanations, an evolutionary perspective can contribute to our understanding of behavior by providing additional, functional accounts of behavior. We provided examples of such ultimate accounts of behavior, and proposed several implications of the evolutionary framework for research in health psychology, and for intervention design. First, we suggested that behavior change interventions can be designed to target, and work in synergy with, functional aspects of behavior. Second, based on our review of the evolutionary psychological literature, we proposed that motivations underlying health behavior likely reflect domainspecific motivational systems - rather than a domain-general motivation to stay healthy. Finally, reviewing the literature centering on the notion of life history strategy, we proposed that this framework can illuminate both 
variation in and covariance between unhealthy behaviors. The reviewed literature also suggests that early-life environments of people (childhood experience) could be a key target for intervention.

Chapter 3 examined one proposed domain-specific motivational system that may help to understand health-protective behaviors. The reported cross-sectional studies in this chapter measured trait-level differences (dispositional variation) in pathogen-avoidance motivation, related to 1) screening behavior, and 2) participants' history of casual sex. More specifically, in Study 1 we used measures of pathogen-avoidance and examined how these related to attitudes and intentions to test for sexuallytransmitted infections (STI) and history of casual sex. Study 2 extended this design - here, we included not only STI screening attitude and intention measures, but tested relationships with the attitude and intention towards various other screening behaviors. We distinguished between screening behaviors for pathogen-related health issues (e.g. STI or rabies screening) and those that involve screening for pathogen-unrelated health concerns (e.g. headaches or getting a mole checked out). Results suggested that pathogenavoidance motivations have two complementary health-protective effects. First, they may reduce inclinations to engage in casual sex. And second, they may improve attitudes and intentions towards health screening behavior thereby potentially bolstering health-protective behaviors. Interestingly, higher levels of pathogen-avoidance tendencies were strongly linked to improved attitudes and intentions towards testing for STI, as well as to a range of both pathogen-related and unrelated screening behaviors. This suggests that individuals with stronger motivations to avoid pathogens are more protective of their health in general - a domain-general effect of a domain-specific motivation.

Chapter 4 examined the role of pathogen-avoidance motivation on STI testing attitudes - and the underlying beliefs shaping this variable- in an experimental set-up. This study used a motivational prime that is designed to trigger a pathogen-avoidance motivational state. In addition to testing the effect of this prime on attitude towards STI testing, we examined how the activation of state-level pathogen-avoidance would affect the beliefs underlying individuals' attitude towards STI testing. Contrary to expectations, the essay prime did not improve overall attitudes toward STI testing, but (in line with predictions) the manipulation did cause some changes in the importance of specific - disease-related - beliefs about the consequences of getting tested for STI. Specifically, we found that in the experimental condition (primed with pathogen-avoidance) the predictive strength of some disease-related beliefs on attitude increased - whereas beliefs with different content (i.e. not related to disease) became less important.

Chapter 5 examined what is so negative about getting tested for STI if indeed it is perceived as such. The studies described in this chapter hypothesized that pathogen-avoidance motivation may - in addition to 
potentially promoting health-protective behavior - also provide insights into an important barrier to STI testing; anticipated negative responses from other people. We examined whether getting tested for STI increases negative attitudes towards vignette descriptions, and whether this negativity is tied to people perceiving individuals who screen for STI as 'infectious' versus 'promiscuous'. Across two studies (cross-sectional and experimental) we found a clear pattern: both perceptions of infectiousness and promiscuity predicted negative attitudes towards and attributed shame to the tester. However, contrary to our expectations, testing for STI did not increase such negativity - but rather decreased negative attitudes and attributed shame.

\section{Theoretical implications}

In chapter 2, the general framework of evolutionary approaches to human behavior was introduced, and how this approach differs from standard theoretical approaches in health psychology was reviewed. An important theoretical aspect discussed in this chapter involved domainspecificity - human psychology evolved gradually, in response to different selective pressures that operated in our past, and therefore human's psychology likely consists of discrete problem-solving mechanisms (Cosmides \& Tooby, 2013). One such recurring problem has been pathogens - infectious microbes - that have posed fitness challenges in our past (and in the present), and therefore are thought to have shaped a domain-specific pathogen-avoidance motivational system (Tybur \& Lieberman, 2016). We found that (in both Studies 1 and 2 of Chapter 3), when measured as an individual differences variable (trait level), pathogen-avoidance motivation positively related to attitude and intention to get tested for STI. Variation in our operationalization of pathogen-avoidance was associated with motivation to test for STI, but also various other screening attitudes and intentions.

What do these associations tell us? First, it is important to note that we used self-report measures of pathogen-avoidance designed to measure proximate aspects of an ultimate motivation to avoid pathogens. The studies reported in Chapter 3 used two such measures, the germ aversion scale (Duncan et al., 2009) and the pathogen disgust facet (Tybur et al., 2009). The germ aversion scale measures participants' self-reported 'aversive affective responses to situations that connote a relatively high likelihood of pathogen transmission' (Duncan et al., 2009, p. 542). For example, the scale uses items such as 'I prefer to wash my hands pretty soon after shaking someone's hand' and 'It really bothers me when people sneeze without covering their mouths'. The pathogen disgust facet was developed to measure trait variation in disgust sensitivity to objects and situations containing cues to pathogen presence. For instance, situations such as 'sitting next to someone with open red sores on their arm' or 'shaking hands with a stranger who has sweaty palms'. We thus captured both affective and cognitive aspects of pathogen-avoidance motivation with these measures. 
While the associations with STI testing attitude and intention were small, larger associations were observed when we predicted a constellation of screening behaviors to pathogen threats. At the same time, negative associations with sociosexuality - individuals' inclination to engage in casual, uncommitted sex - were observed. We suggest that these results are in line with the notion that specific psychological mechanisms regulate avoidance of pathogens - which involves tradeoffs (e.g., Murray et al., 2013; Mark Schaller \& Park, 2011; Tybur et al., 2013). For those individuals high on pathogen-avoidance motivation, one would expect to observe behavioral attitudes and intentions that are in line with this motivation - an orientation towards activities which may alleviate pathogen threats, such as getting screened. Similarly, one would also expect inclinations to avoid activities that increase the probability of contracting pathogens - such as uncommitted sexual behavior. Indeed, our results revealed precisely this: a decreased interest in casual sex and an increased tendency to screen for infectious diseases. Thus, we suggest that this study revealed two healthprotective effects relating to pathogen-avoidance - both decreased interest in casual sex and increased inclinations to screen for infectious disease.

The results of the studies reported in Chapter 3, however, also indicated that a large percentage of the variance in pathogen-unrelated screening attitudes and intentions was explained by pathogen-avoidance motivation. While there was some difference in the proportion explained variance between pathogen-related screening and pathogen-unrelated screening (58\% versus 46\%), we expected that this difference would have been larger. This can be explained by considering a signal detection problem involved in reacting to pathogens. That is, authors have proposed that detection of pathogens - given the impossibility to detect these directly proceeds through detection of pathogen cues (Tybur \& Lieberman, 2016). It has further been argued that such detection of pathogen cues likely exhibits adaptive biases - a tendency to 'over-perceive' cues connoting pathogen presence (Neuberg et al., 2011; Mark Schaller \& Park, 2011; Tybur \& Lieberman, 2016; Tybur et al., 2013). One functional explanation for the association between pathogen-avoidance motivation and pathogen-unrelated screening attitudes and intentions we found in our research, then, may be that a tendency to monitor pathogen cues generates 'false-positives'. Hence, individual differences in pathogen-avoidance motivation may become apparent in responses to symptoms such as headaches, fatigue, or a mole. This suggests that people high on pathogen-avoidance treat any symptoms more readily as indicative of pathogens. Alternatively, it is possible that the relationships exist because people high on pathogen-avoidance motivation have a heightened overall inclination to check out potential health issues. Thus, pathogen-avoidance motivation may be a domain-specific motivation with more generalized health-protective functions. The connection between pathogen-avoidance and health-protective functions is underscored by literature on psychopathology and its relation to disgust. For example, 
disgust (as a key motivational driver of pathogen-avoidance) has been linked to obsessive-compulsive disorder, hypochondriasis and various other extreme health anxiety (Cisler, Olatunji, \& Lohr, 2009; Davey, 2011; Sprengelmeyer et al., 1997). These associations provide further evidence for the importance of pathogen-avoidance motivation to understand health behavior.

In addition to examining individual differences in pathogen-avoidance motivation, the research reported in Chapter 4 attempted to induce change in state-levels of this motivation (i.e. temporarily activated). To this end, we primed pathogen disgust by having participants read an essay that vividly described pathogen-disgust provoking events, the effect of which was compared to a control group who were given a neutral essay to read instead. The effects of this manipulation were unclear yet provoking - while we observed some changes on the belief level (i.e. some disease related behavioral beliefs became more important in the experimental group), there was no overall change in participants' attitude towards STI testing. Two explanations are likely for the absence of an effect on attitude, which could explain why we did observe changes in the underlying belief structure but not the attitude variable itself. First, the overall scores on attitude towards STI were highly skewed towards highly positive scores - this ceiling effect may have limited the potential of our manipulation to further increase these scores. Second, the manipulation used may not have elicited sufficient level of pathogen disgust for our measurement of attitude to be affected by such a motivation.

Although no differences between groups were found for overall attitudes, the finding that specific behavioral beliefs did change in the strength with which they predicted attitude was partially in line with our hypotheses. We found that the pathogen-avoidance manipulation led to changes in how the importance of specific beliefs predicted participants' attitude - specifically, the importance of the belief that 'getting tested for STI would reduce feelings of anxiety/fear of having contracted an STI' became more important due to the pathogen-avoidance manipulation. Additionally, the importance of the belief that 'getting tested for STI would cause stress and worry due to the possibility of testing positive' was attenuated due to the manipulation. Thus, these results provide some suggestion that a pathogen-avoidance motivational state - here, by triggering pathogen disgust - may influence the importance of specific behavioral beliefs.

Our findings that beliefs about specific consequences are influenced by state level motivational states - if correct - have important implications for health psychological research. It raises the question whether beliefs can be considered as static, or stable, across situations, environments, and contexts. A central task in developing an effective behavior change intervention rests on identifying the determinants of behavior (Bartholomew Eldregde et al., 2016), which include the beliefs people have about the consequences of behavior. The relevance of these beliefs is, however, 
usually measured and charted independent of the behavioral context (e.g., through survey research). The results reported in Chapter 4 suggest that motivational context may have consequences on decisions about which beliefs to target with interventions. This notion is line with research that targeted a different fundamental motivation for behavior (Chiou et al., 2015; S. E. Hill \& Durante, 2011). One of these studies (S. E. Hill \& Durante, 2011) found that activating (and thereby prioritizing) a mating motivation decreased women's perceived susceptibility to the negative consequences of tanning and diet pills, whereas a similar paradigm was used to examine the effect of mating motivation on males' self-control over smoking (Chiou et al., 2015). In this latter study, male smokers exposed to photos of attractive women were more likely to discount future health concerns, and instead gave in to their immediate impulse to smoke, indicating a loss of self-control. Our findings are in line with these studies suggesting that momentarily activation of a domain-specific motivation -be it pathogen avoidance or mating - has subsequent effects on cognition and behavior. This is in line with ideas forwarded in - for example - the fundamental motives framework. Thus, we conclude that understanding the very basic, fundamental motivations (see Kenrick, Neuberg, et al., 2010; Mark Schaller et al., 2017) and how and when they can help understand behavior - may contribute to our understanding of health behavior.

Our examination of pathogen-avoidance motivation also took another approach - specifically, how this motivation affects interpersonal behavior, and how it may help understand barriers to STI testing. In the studies reported in Chapter 5 we first investigated whether STI testing is related to negative attitudes and attributed shame, and - if so - whether this negativity relates to a perceived infectiousness or promiscuity threat. We expected that merely getting tested for STI would already evoke negative responses by individuals, in that this behavior would be associated with increased perceptions of infectiousness and promiscuity. We found no evidence for this idea, and across two studies we found that STI testing decreases negativity to the targets described in the vignettes. This is against the expectation that getting screened for STI would trigger diseaseassociations, and would affect contamination beliefs regarding the target which in turn would be expected to lead to increased perceptions of infectiousness and more negative attitudes towards the testee (Oaten et al., 2011). Nevertheless, although negativity towards individuals getting tested for STI was not found, we did find both correlational and experimental evidence that infectiousness and promiscuity both cause negativity towards others. These findings are line with the literature suggesting that stigmatization is related to regulation of 'social threats', including individuals perceived to impose pathogen-infection risks (Kurzban \& Leary, 2001; Neuberg et al., 2011, 2000). Moreover, promiscuous behavior is often negatively evaluated by others (Bleske \& Schackelford, 2001) -one proposed functional account of this relationship is that promiscuity may be 
threatening to those pursuing monogamous relationships (Kurzban et al., 2010; Tybur et al., 2015). This explanation can be tested in future research that include measures of observers' own sociosexuality (relatively monogamous versus promiscuous) to see if the proposed moderation is indeed the case.

The unanticipated finding that testing for STI does not lead to negative responses by other people is important. It suggests that there is a discrepancy between people's own expectations about the negative social consequences of getting tested - often found to be a barrier to testing for STI (Cunningham et al., 2009; Fortenberry et al., 2002) - and other people's perceptions. Thus, in a sense, our findings suggest that anticipated stigma associated with getting tested for STI may be an instance of 'unrealistic pessimism'.

\section{Methodological evaluation}

In this section, we evaluate the studies on their methodological rigor, appropriateness, and we address some limitations of the studies reported in this dissertation. Chapter 3 described cross-sectional research using selfreport methodology to examine links between pathogen-avoidance motivation and health-protective inclinations. One strength of these studies is that we modelled the relationships between these constructs using structural equation modelling. This methodology has several advantages over OLS regression analyses. First, these models included latent variables to test relationships between constructs, as opposed to using observed scores on variables that assume variables are measured with perfect reliability (Bollen, 2002; MacCallum \& Austin, 2000). Since perfect measurement is usually or always not the case, regular methods of regression are prone to arriving at biased - or attenuated - estimates of relationships between constructs; latent variable models take such measurement error into account while estimating relationships.

The studies reported in Chapter 3 also have several limitations, the most important one relating to the concept that correlation does not imply causation expression. That is, because the included variables were all measured - as opposed to manipulated - the directionality of the relationships specified in these models is tentative. While the relationships were specified in line with theory, no conclusions about directionality can be drawn from this design. Nonetheless, based on theoretical considerations, we suggest that the reported associations imply that pathogen-avoidance mechanisms causally influence health-protective behavior: By both shaping preferences towards restricted, committed sexual relationships, and increasing attitudes and intentions towards STI testing. In other words, by reducing promiscuity and infectiousness. Without follow-up work, it is difficult to exclude alternative directionality or the possible influence of unmeasured variables. 
Across all empirical studies reported in this dissertation, self-report measures were often used as primary outcome or predictor variables. A potential methodological problem when using self-report involves common method bias (Lindell \& Whitney, 2001; Podsakoff, MacKenzie, Lee, \& Podsakoff, 2003). That is, when constructs are measured using similar methods, correlation between two constructs may arise due to the method, instead of a relationship between the constructs. Common method bias in self-report can arise from several sources (for a review; Podsakoff et al., 2003), including having the same respondents rating all measures (common rater effects, e.g., social desirability), or characteristics of the items (e.g. similar scale anchors or demand characteristics). While we did control for some of these effects in the design of our self-report measures (e.g., through randomization) the possibility remains that at least some covariance may be attributable to common method variance.

Additionally, the study reported in Chapter 5, which focused on the effect of getting tested for STI on others' negative attitudes and attributed shame, may specifically have suffered from social desirability. In particular, the explicit measurement of these outcomes (negative attitudes and attributed shame) potentially invite responses in the direction of 'what would be normative to answer', i.e. that someone who gets tested for STI should be evaluated positively (cf. Dovidio, Kawakami, Johnson, Johnson, \& Howard, 1997). In the context of research topics that are prone to social desirability (e.g. stigma or sexuality research), to circumvent bias researchers have used methods such as implicit association tasks (IAT) to tap into attitudes, or instead relied on other indirect (behavioral or physiological) indicators. The results reported in Chapter $\mathbf{5}$ which suggest that getting tested does not in itself cause negativity, would benefit greatly from replication and extension, for example by using indirect measures (see Fazio \& Olson, 2003).

In Chapter 4 we aimed to pursue the relationship between pathogenavoidance motivation and STI screening attitude in an experimental set-up. The manipulation we used was an essay describing pathogen disgust provoking events. This manipulation had been shown to successfully induce pathogen-avoidance motivation by eliciting disgust in previous research (White et al., 2013). In the current study, while finding differences in the importance of specific beliefs, no overall differences on testing attitude were observed.

The work is also limited by the use of determinants (attitude and intention) as primary outcome variables. While various meta-analyses show that these variables causally relate to actual behavior (Sheeran et al., 2016; T. L. Webb \& Sheeran, 2006), the relationships between determinants and behavior is attenuated by moderating variables (e.g. people's ability to actually perform a behavior). The use of behavioral outcome measures, therefore, would further strengthen the reported findings. Overall, then, across these studies, while finding some evidence that pathogen-avoidance relates to health-protective attitudes, more research with varying methods is 
needed to firmly establish the health-protective effects of pathogenavoidance motivation. In a later section, I will return to suggestions for future studies.

\section{What do evolutionary perspectives add? (and what don't they add?)}

This dissertation examined the added value of evolutionary perspectives on health behavior, by focusing in particular on one proposed healthrelevant motivation: the motivation to avoid pathogens. While finding some empirical support for the idea that this domain-specific motivation may be important to understand health behavior, a more abstract question remains - can future research in health psychology benefit by insights gained from evolutionary psychology by applying it as a meta-theory? Or can we easily go without it?

Discussion of this question in Chapter 2 led to identifying the added value of evolutionary psychology in terms of an explanatory perspective - the possibility to construct and test functional (ultimate) hypotheses about behavior (Scott-Phillips et al., 2011). For example, Chapter 2 illustrated that risk taking can be functionally understood as a behavioral analogue of the peacock's tail, and we reviewed examples of how such hypotheses have been tested (e.g., Hone et al., 2013; Ronay \& Hippel, 2010). The metatheoretical potential of evolutionary theory was further illustrated in Chapter 2 by using the dubious notion that people, all else being equal, can be expected to pursue health goals - an assumption that evolutionary theory not easily justifies. Further, it has also been discussed that, in principle, such ultimate explanations are independent of proximate explanations aiming to understand the causal mechanisms underlying behavior knowing the ultimate why of behavior is not tantamount to knowing how it occurs (see also the opening-citation at page 28). Therefore, health psychologists may - in principle - learn everything there is to know about the psychological processes underlying behavior, without any reference to ultimate explanations. So, why do we need to consider them?

A concrete example can help answer this question. In several chapters we have referred to two studies (Meertens et al., 2013; Tybur et al., 2011) that provided an empirical backdrop to our hypotheses concerning the health-protective effects of pathogen-avoidance motivation. To recap these studies, Tybur et al. (Tybur et al., 2011) invited participants into a lab that was sprayed with an odor similar to those associated with pathogen sources. It was hypothesized that this olfactory cue to pathogens would motivate prophylactic behavior - specifically, that it would increase participants' intention to use, buy, and discuss the use of condoms in future sexual encounters. Meertens et al. (Meertens et al., 2013) examined the effect of the physical environment on participants' beliefs about susceptibility to STI. They found that participants who imagined waking up in a dirty room after a one night stand judged their susceptibility to STI to be higher than did participants who imagined waking up in a clean environment after a one 
night stand. Participants imagining the dirty room also considered the odds of engaging in unsafe casual sex in the future significantly lower.

While the study by Tybur et al (2011) explicitly generated hypotheses from an evolutionary perspective on pathogen-avoidance, and tested whether a stinky smell (functionally expected to cue pathogen presence) would influence condom use intentions, the study by Meertens et al. (2013) did not use this theoretical perspective. These authors instead tested the effects of a clean versus dirty environment on perceived susceptibility through alterations in mood. These studies capture the distinction made in the introduction of this dissertation between a functional perspective (dirty smell cue pathogen disgust which motivates pathogen-avoidance) versus a process perspective (a clean physical environment leads to optimistic perceptions of risks through inducing a positive mood - and the inverse). Interestingly, the hypotheses tested by Meertens et al. (2013) could have been directly drawn from work on pathogen-avoidance and pathogen disgust (e.g., Curtis et al., 2004, 2011; Oaten et al., 2009; Tybur et al., 2013), but nevertheless - based on a different literature - these authors made similar predictions on how the cleanliness of the environment would influence health-protective intentions. Indeed, a critical health psychologist may ask what the functional perspective taken by Tybur et al (2013) adds to the proximate, process perspective taken by Meertens et al (2011).

We suggest that consideration of the ultimate, functional aspects of behavior may not necessarily generate unique study hypotheses - similar hypotheses can be arrived at from many different levels of analyses. However, the manner in which researchers arrive at hypotheses may have consequences for the interpretation of the findings, and for the direction of follow-up hypotheses. Consider the difference in interpretation of the effect of a dirty environment. Within the framework proposed by Meertens et al, negative affect was one psychological variable potentially responsible for the observed effect on perceived susceptibility to STI (although 'magical contamination' was discussed as an alternative). In the Tybur et al study, the functional explanation for the observed effect focused on the proposal that specific perceptual cues which are reliably associated with pathogen presence motivate preventative actions. From a negative affect caused by environmental cues perspective, one could hypothesize that any means of environmentally induced negative affect after engaging in unsafe sexual practices would increase perceived susceptibility and intentions to engage in unsafe sex again. For example, this would arguably also generate the prediction that participants who imagine waking up in a room stocked with weaponry, after having unsafe sex, would report higher perceived susceptibility to STI and decreased intentions to repeat the behavior. Considered from the functional perspective of pathogen-avoidance, however, this prediction would not follow - since not the negative affect would be central to understanding perceived susceptibility and intentions, but the nature of the threat (here, pathogens) would be critical in influencing 
behavior. Overall, evolutionary psychology's focus on domain-specific motivations, regulating responses to tangible threats and opportunities, may thus help to shape more specific hypotheses about health behavior - and, by doing so, provide added value to current health behavior theory. Thus, while in principle it is possible to identify all the factors that motivate health behavior without any reference to evolutionary theory, consideration of ultimate reasons for behavior may help to shape, refine, and help deduce novel hypotheses.

\section{Future research}

This dissertation has focused on applications of pathogen-avoidance motivation to understand health-protective behaviors. The work reported across the various chapters leave ample room for improvement through replication (see methodological evaluation), follow-up, and extension. For instance, the various evolutionary models of motivation - providing domainspecific predictions of the structure of motivation - specify more potentially health-relevant motivational systems (Aunger \& Curtis, 2013; Bernard et al., 2005; Mark Schaller et al., 2017). Each of the motivational systems specified within these frameworks can be expected to relate to health behavior in specific ways, and provide alternatives to the assumption of a domaingeneral health motivation. Thus, by looking at the kinds of motivations expected to be engrained in our evolved psychology, we may come to a better understanding of the threats and opportunities people are likely to act upon.

To illustrate, the evolutionary psychological literature proposes various other discrete motivational systems likely to have evolved in response to specific fitness-relevant problems. These include, for example, motivations to maintain alliances in groups, raising offspring, acquiring mates, and avoiding physical harm (e.g., Kenrick, Neuberg, et al., 2010). One line of research has, for example, targeted motivations thought to be related to adaptive problems of group living, and the needs to affiliate and maintain alliances. Working from the idea that humans have historically been dependent on group living, evolutionary psychologists hypothesized that people likely exhibit domain-specific mechanisms that are sensitive to threats and opportunities involving status and reputation (Griskevicius, Tybur, \& Van den Bergh, 2010; Hardy \& Van Vugt, 2006; van Vugt \& Hardy, 2009). Some of this work has shown that when concerns about reputation come into play, people tend to make more prosocial decisions that go against self-interest. For instance, Van Vugt and Hardy (2010) showed that people engage in conspicuous contributions (e.g., make unnecessary contributions to a public good) when reputation concerns come into play. Griskevicius, Tybur, and Van den Bergh (2010) showed that concerns about status and reputation also influenced conspicuous conservation behavior - participants primed with a status motive were more inclined to buy a green product over a more luxurious non-green product. Health psychology research may also benefit from utilizing such status and reputation concerns to stimulate 
health-protective behaviors - through tactics that make such specific social consequences of health-protective behaviors salient. For example, one could focus on how screening for disease not only relates to pathogen concerns, but also how others may perceive someone's preventative behavior.

\section{Conclusion}

The findings reported in this dissertation suggest that pathogenavoidance motivation may help us understand health behavior, although more research is needed to firmly establish the relevance of pathogenavoidance processes to understand health behavior. More generally, this dissertation has illustrated how evolutionary perspectives can add to current health behavior theory - by serving a meta-theoretical role enabling the generation of novel hypotheses. This dissertation is just a first and modest step towards a more substantial integration of health psychology and evolutionary approaches. The biologist Dobzhansky remarked that 'nothing in biology makes sense, except in the light of evolution'. In a similar vein, we conclude that nothing about people's health behavior - both the health detrimental and health promoting ones - makes sense, except in the light of evolution. 

REFERENCES 
Ahlstrom, B., Dinh, T., Haselton, M. G., \& Tomiyama, A. J. (2017). Understanding eating interventions through an evolutionary lens. Health Psychology Review, 11(1), 72-88. https://doi.org/10.1080/17437199.2016.1260489

Ajzen, I. (1985). From intentions to actions: A theory of planned behavior. (J. Kuhl \& J. Beckmann, Eds.), Action Control. Springer. https://doi.org/10.1007/978-3-64269746-3_2

Ajzen, I. (1991). The theory of planned behavior. Organizational Behavior and Human Decision Processes, 50(2), 179-211. https://doi.org/10.1016/0749-5978(91)90020-T

Ajzen, I., \& Fishbein, M. (2008). Scaling and testing multiplicative combinations in the expectancy-value model of attitudes. Journal of Applied Social Psychology, 38(9), 2222 2247. https://doi.org/10.1111/j.1559-1816.2008.00389.x

Albarracin, D., Johnson, B. T., Fishbein, M., \& Muellerleile, P. A. (2001). Theories of reasoned action and planned behavior as models of condom use: a meta-analysis. Psychological Bulletin, 127(1), 142.

Alessi, G. (1992). Models of proximate and ultimate causation in psychology. The American Psychologist, 47(11), 1359-1370. https://doi.org/10.1037/0003-066X.47.11.1359

Anderson, N. B. (1998). Levels of Analysis in Health Science: A Framework for Integrating Sociobehavioral and Biomedical Research. Annals of the New York Academy of Sciences, 840(1), 563-576. https://doi.org/10.1111/j.1749-6632.1998.tb09595.x

Anokhin, A. P., Grant, J. D., Mulligan, R. C., \& Heath, A. C. (2015). The Genetics of Impulsivity: Evidence for the Heritability of Delay Discounting. Biological Psychiatry, 77(10), 887-894. https://doi.org/10.1016/j.biopsych.2014.10.022

Arndt, J., \& Goldenberg, J. L. (2017). Where Health and Death Intersect. Current Directions in Psychological Science, 26(2), 126-131. https://doi.org/10.1177/0963721416689563

Aunger, R., \& Curtis, V. (2013). The Anatomy of Motivation: An Evolutionary-Ecological Approach. Biological Theory, 8(1), 49-63. https://doi.org/10.1007/s13752-013-0101-7

Aunger, R., \& Curtis, V. (2016). Behaviour Centred Design: towards an applied science of behaviour change. Health Psychology Review, 10(4), 425-446. https://doi.org/10.1080/17437199.2016.1219673

Bailey, J. M., Kirk, K. M., Zhu, G., Dunne, M. P., \& Martin, N. G. (2000). Do individual differences in sociosexuality represent genetic or environmentally contingent strategies? Evidence from the Australian twin registry. Journal of Personality and Social Psychology, 78(3), 537-545. https://doi.org/10.1037/0022-3514.78.3.537

Balfe, M., Brugha, R., O'Connell, E., McGee, H., O’Donovan, D., \& Vaughan, D. (2010). Why don't young women go for Chlamydia testing? A qualitative study employing Goffman's stigma framework. Health, Risk \& Society, 12(2), 131-148. https://doi.org/10.1080/13698571003632437

Barrett, H. C. (2012). A hierarchical model of the evolution of human brain specializations. Proceedings of the National Academy of Sciences of the United States of America, 2012, 1-8. https://doi.org/10.1073/pnas.1201898109

Barrett, H. C., \& Kurzban, R. (2006). Modularity in cognition: Framing the debate. Psychological Review, 113(3), 628-647. https://doi.org/10.1037/0033295X.113.3.628

Barth, K. R., Cook, R. L., Downs, J. S., Switzer, G. E., \& Fischhoff, B. (2002). Social stigma and negative consequences: factors that influence college students' decisions to seek testing for sexually transmitted infections. Journal of American College Health: $J$ of ACH, 5O(4), 153-159. https://doi.org/10.1080/07448480209596021

Bartholomew Eldregde, L. K., Markham, C. M., Ruiter, R. A. C., Fernàndez, M. E., Kok, G., Parcel, G. S., ... Parcel, G. S. (2016). Planning health promotion programs: An Intervention Mapping approach (4th ed.). Hoboken, NJ: Wiley.

Bateson, P., \& Laland, K. N. (2013). Tinbergen's four questions: An appreciation and an update. Trends in Ecology and Evolution, 28(12), 712-718.

https://doi.org/10.1016/j.tree.2013.09.013 
Belsky, J., Schlomer, G., \& Ellis, B. (2012). Beyond cumulative risk: Distinguishing harshness and unpredictability as determinants of parenting and early life history strategy. Developmental Psychology, 48(3), 662-673. https://doi.org/10.1037/a0025837

Bernard, L. C., Mills, M., Swenson, L., \& Walsh, R. P. (2005). An evolutionary theory of human motivation. Genetic Social and General Psychology Monographs, 131(2), 129184. https://doi.org/Doi 10.3200/Mono.131.2.129-184

Biran, A., Schmidt, W.-P. P., Varadharajan, K. S., Rajaraman, D., Kumar, R., Greenland, K., ... Curtis, V. (2014). Effect of a behaviour-change intervention on handwashing with soap in India (SuperAmma): a cluster-randomised trial. Lancet Global Health, 2(3), E145-E154. https://doi.org/10.1016/S2214-109X(13)70160-8

Bleske, A. L., \& Schackelford, T. K. (2001). Poaching, promiscuity, and deceit: Combatting mating rivalry in same-sex friendships. Personal Relationships, 8(4), 407-424. https://doi.org/10.1111/j.1475-6811.2001.tb00048.x

Bollen, K. A. (2002). Latent variables in psychology and the social sciences. Annual Review of Psychology, 53, 605-34.

https://doi.org/10.1146/annurev.psych.53.100901.135239

Borg, C., \& de Jong, P. J. (2012). Feelings of Disgust and Disgust-Induced Avoidance Weaken following Induced Sexual Arousal in Women. Plos One, 7(9). https://doi.org/ARTN e44111. DOI 10.1371/journal.pone.0044111

Bos, A. E. R., Pryor, J. B., Reeder, G. D., \& Stutterheim, S. E. (2013). Stigma: Advances in Theory and Research. Basic and Applied Social Psychology, 35(1), 1-9. https://doi.org/10.1080/01973533.2012.746147

Brennan, S. L., Henry, M. J., Nicholson, G. C., Kotowicz, M. A., \& Pasco, J. A. (2009). Socioeconomic status and risk factors for obesity and metabolic disorders in a population-based sample of adult females. Preventive Medicine, 49(2-3), 165-171. https://doi.org/10.1016/j.ypmed.2009.06.021

Bryan, A. D., Aiken, L. S., \& West, S. G. (1996). Increasing condom use: evaluation of a theory-based intervention to prevent sexually transmitted diseases in young women. Health Psychology, 15(5), 371-382. https://doi.org/Doi 10.1037/0278-6133.15.5.371

Buchner, A., Bell, R., Mehl, B., \& Musch, J. (2009). No enhanced recognition memory, but better source memory for faces of cheaters. Evolution and Human Behavior, 30(3), 212 224. https://doi.org/10.1016/j.evolhumbehav.2009.01.004

Buhrmester, M., Kwang, T., \& Gosling, S. D. (2011). Amazon's Mechanical Turk a new source of inexpensive, yet high-quality, data? Perspectives on Psychological Science, 6(1), 3-5. https://doi.org/Doi 10.1177/1745691610393980

Burke, D. (2014). Why isn't everyone an evolutionary psychologist? Frontiers in Psychology, 5(AUG), 1-8. https://doi.org/10.3389/fpsyg.2014.00910

Buss, D. M. (1997). Human Social Motivation in Evolutionary Perspective: Grounding Terror Management Theory. Psychological Inquiry, 8(1), 22-26.

https://doi.org/10.1207/s15327965pli0801_3

Buss, D. M. (2009). How Can Evolutionary Psychology Successfully Explain Personality and Individual Differences? Perspectives on Psychological Science, 4(4), 359-366. https://doi.org/10.1111/j.1745-6924.2009.01138.x

Casey, B. J., Jones, R. M., \& Hare, T. A. (2008). The adolescent brain. Annals of the New York Academy of Sciences. https://doi.org/10.1196/annals.1440.010

Champagne, F. A., \& Mashoodh, R. (2009). Genes in Context: Gene-Environment Interplay and the Origins of Individual Differences in Behavior. Current Directions in Psychological Science, 18(3), 127-131. https://doi.org/10.1111/j.14678721.2009.01622.x

Chen, P., \& Vazsonyi, A. T. (2013). Future Orientation, School Contexts, and Problem Behaviors: A Multilevel Study. Journal of Youth and Adolescence, 42(1), 67-81. https://doi.org/10.1007/s10964-012-9785-4 
Chiou, W.-B., Wu, W.-H., \& Cheng, Y.-Y. (2015). Beauty against tobacco control: viewing photos of attractive women may induce a mating mindset, leading to reduced selfcontrol over smoking among male smokers. Evolution and Human Behavior, 36(3), 218 223. https://doi.org/10.1016/j.evolhumbehav.2014.11.006

Cisler, J. M., Olatunji, B. O., \& Lohr, J. M. (2009). Disgust, fear, and the anxiety disorders: A critical review. Clinical Psychology Review, 29(1), 34-46. https://doi.org/10.1016/j.cpr.2008.09.007

Cooper, M. D., \& Alder, M. N. (2006). The evolution of adaptive immune systems. Cell, 124(4), 815-822. https://doi.org/10.1016/j.cell.2006.02.001

Cosmides, L. (1989). The logic of social exchange: Has natural selection shaped how humans reason? Studies with the Wason selection task. Cognition, 31(3), 187. https://doi.org/10.1016/0010-0277(89)90023-1

Cosmides, L., \& Tooby, J. (1996). Are humans good intuitive statisticians after all? Rethinking some conclusions from the literature on judgment under uncertainty. Cognition, 58(1), 1-73. https://doi.org/10.1016/0010-0277(95)00664-8

Cosmides, L., \& Tooby, J. (2013). Evolutionary Psychology: New Perspectives on Cognition and Motivation. Annual Review of Psychology, 64(1), 201-229. https://doi.org/doi:10.1146/annurev.psych.121208.131628

Cosmides, L., Tooby, J., \& Kurzban, R. (2003). Perceptions of race. Trends in Cognitive Sciences, 7(4), 173-179. https://doi.org/10.1016/S1364-6613(03)00057-3

Cottrell, C. A., \& Neuberg, S. L. (2005). Different Emotional Reactions to Different Groups: A Sociofunctional Threat-Based Approach to "Prejudice". Journal of Personality and Social Psychology, 88(5), 770-789. https://doi.org/10.1037/0022-3514.88.5.770

Cottrell, C. A., Neuberg, S. L., \& Li, N. P. (2007). What do people desire in others? A sociofunctional perspective on the importance of different valued characteristics. Journal of Personality and Social Psychology, 92(2), 208-231. https://doi.org/10.1037/0022-3514.92.2.208

Crawford, M., \& Popp, D. (2003). Sexual double standards: A review and methodological critique of two decades of research. Journal of Sex Research, 4O(1), 13-26. https://doi.org/10.1080/00224490309552163

Cunningham, S. D., Kerrigan, D. L., Jennings, J. M., \& Ellen, J. M. (2009). Relationships Between Perceived STD-Related Stigma, STD-Related Shame and STD Screening Among a Household Sample of Adolescents. Perspectives on Sexual and Reproductive Health, 41(4), 225-230. https://doi.org/10.1363/4122509

Curtis, V. (2011). Why disgust matters. Philosophical Transactions of the Royal Society of London. Series B, Biological Sciences, 366(1583), 3478-90. https://doi.org/10.1098/rstb.2011.0165

Curtis, V. (2013). Don't Look, Don't Touch, Don't Eat: The Science Behind Revulsion. Chicago: University of Chicago Press.

Curtis, V., Aunger, R., \& Rabie, T. (2004). Evidence that disgust evolved to protect from risk of disease. Proceedings: Biological Sciences (Biology Letters), 277, 131-133. https://doi.org/10.1098/rsbl.2003.0144

Curtis, V., de Barra, M., \& Aunger, R. (2011). Disgust as an adaptive system for disease avoidance behaviour. Philosophical Transactions of the Royal Society of London. Series B, Biological Sciences, 366(1563), 389-401. https://doi.org/10.1098/rstb.2010.0117

Darwin, C. . (1859). On the origin of species by means of natural selection. New York: Gramercy books.

Davey, G. C. L. (2011). Disgust: the disease-avoidance emotion and its dysfunctions. Philosophical Transactions of the Royal Society of London B: Biological Sciences, 366(1583), 3453-3465. Retrieved from

http://rstb.royalsocietypublishing.org/content/366/1583/3453.abstract

Davis, R., Campbell, R., Hildon, Z., Hobbs, L., \& Michie, S. (2015). Theories of behaviour and behaviour change across the social and behavioural sciences: a scoping review. 
Health Psychology Review, 9(3), 323-344.

https://doi.org/10.1080/17437199.2014.941722

Dawkins, R. (1976). The Selfish Gene. Oxford, U.S.: Oxford University Press.

Dawkins, R. (2009). The Greatest Show on Earth: The evidence for evolution. New York, US.: Free press.

de Vries, H. (2017). Thinking is the best way to travel: towards an ecological interactionist approach; a comment on Peters and Crutzen. Health Psychology Review, O(0), 1-6. https://doi.org/10.1080/17437199.2017.1306719

de Vries, H., Mesters, I., Steeg, H. van de, \& Honing, C. (2005). The general public's information needs and perceptions regarding hereditary cancer: an application of the Integrated Change Model. Patient Education and Counseling, 56(2), 154-165. https://doi.org/10.1016/j.pec.2004.01.002

Dovidio, J. F., Kawakami, K., Johnson, C., Johnson, B., \& Howard, A. (1997). On the Nature of Prejudice: Automatic and Controlled Processes. Journal of Experimental Social Psychology, 33(5), 510-540. https://doi.org/10.1006/jesp.1997.1331

Dunbar, R. I. M. (1998). The Social Brain Hypothesis. Evolutionary Anthropology, 178-190. https://doi.org/10.1002/(SICI) 1520-6505(1998)6:5<178::AID-EVAN5>3.3.CO;2-P

Dunbar, R. I. M., Shultz, S., Press, H., York, N., \& Nw, A. (2007). Evolution in the Social Brain. Science, 317(5843), 1344-1347. https://doi.org/10.1126/science.1145463

Duncan, L. A., Schaller, M., \& Park, J. H. (2009). Perceived vulnerability to disease: Development and validation of a 15-item self-report instrument. Personality and Individual Differences, 47(6), 541-546. https://doi.org/10.1016/j.paid.2009.05.001

Dunn, T. J., Baguley, T., \& Brunsden, V. (2014). From alpha to omega: A practical solution to the pervasive problem of internal consistency estimation. British Journal of Psychology, 105(3), 399-412. https://doi.org/10.1111/bjop.12046

Ellis, B. J., Del Giudice, M., Dishion, T. J., Figueredo, A. J., Gray, P., Griskevicius, V., ... Volk, A. A. (2012). The evolutionary basis of risky adolescent behavior: implications for science, policy, and practice. Developmental Psychology, 48(3), 598.

Ellis, B. J., Figueredo, A. J., Brumbach, B. H., \& Schlomer, G. L. (2009). Fundamental Dimensions of Environmental Risk. Human Nature (Vol. 20). https://doi.org/10.1007/s12110-009-9063-7

Faulkner, J., Schaller, M., Park, J. H., \& Duncan, L. A. (2004). Evolved Disease-Avoidance Mechanisms and Contemporary Xenophobic Attitudes. Group Processes \& Intergroup Relations, 7(4), 333-353. https://doi.org/10.1177/1368430204046142

Fazio, R. H., \& Olson, M. A. (2003). Implicit Measures in Social Cognition Research: Their Meaning and Use. Annual Review of Psychology, 54(1), 297-327. https://doi.org/10.1146/annurev.psych.54.101601.145225

Figueredo, A. J., Cabeza de Baca, T., Woodley, M. A., de Baca, T., \& Woodley, M. A. (2012). The measurement of Human Life History strategy. Personality and Individual Differences, 55(3), 251-255. https://doi.org/10.1016/j.paid.2012.04.033

Figueredo, A. J., Vasquez, G., Brumbach, B. H., \& Schneider, S. M. R. (2007). The K-factor, Covitality, and personality. Human Nature, 18(1), 47-73. https://doi.org/10.1007/BF02820846

Figueredo, A. J., Vasquez, G., Brumbach, B. H., Sefcek, J. A., Kirsner, B. R., \& Jacobs, W. J. (2005). The K-factor: Individual differences in life history strategy. Personality and Individual Differences, 39(8), 1349-1360. https://doi.org/10.1016/j.paid.2005.06.009

Fishbein, M. (2008). A reasoned action approach to health promotion. Medical Decision Making: An International Journal of the Society for Medical Decision Making, 28(6), 83444. https://doi.org/10.1177/0272989X08326092

Fishbein, M., \& Ajzen, I. (1975). Belief, attitude, attitude, intention and behavior: An introduction to theory of research. Reading, MA : Addison-Wesley Addison-Wesley, 578.

Fishbein, M., \& Ajzen, I. (2010). Predicting and changing behaviour: The reasoned action approach. New York: Taylor and Francis. 
Floyd, D. L., Prentice-Dunn, S., \& Rogers, R. W. (2000). A Meta-analysis of research on protection motivation theory. Journal of Applied Social Psychology.

https://doi.org/10.1111/j.1559-1816.2000.tb02323.x

Fortenberry, J. D. (1997). Health care seeking behaviors related to sexually transmitted diseases among adolescents. American Journal of Public Health, 87(3), 417-420. https://doi.org/10.2105/ajph.87.3.417

Fortenberry, J. D., McFarlane, M., Bleakley, A., Bull, S., Fishbein, M., Grimley, D. M., ... Stoner, B. P. (2002). Relationships of Stigma and Shame to Gonorrhea and HIV Screening. American Journal of Public Health, 92(3), 378-381. https://doi.org/10.2105/AJPH.92.3.378

Francis, R. C. (1990). Causes, proximate and ultimate. Biology and Philosophy, 5(4), 401415. https://doi.org/10.1007/BF02207379

Frankenhuis, W. ., Panchanathan, K., \& Nettle, D. (2016). Cognition in harsh and unpredictable environments. Current Opinion in Psychology. https://doi.org/10.1016/j.copsyc.2015.08.011

Frankenhuis, W. E., \& de Weerth, C. (2013). Does Early-Life Exposure to Stress Shape or Impair Cognition? Current Directions in Psychological Science, 22(5), 407-412. https://doi.org/10.1177/0963721413484324

French, D. P., \& Hankins, M. (2003). The expectancy-value muddle in the theory of planned behaviour - and some proposed solutions. British Journal of Health Psychology, 8(1), 37-55. https://doi.org/10.1348/135910703762879192

Gangestad, S. W., \& Grebe, N. M. (2014). Pathogen avoidance within an integrated immune system: Multiple components with distinct costs and benefits. Evolutionary Behavioral Sciences, 8(4), 226-234. https://doi.org/10.1037/ebs0000023

Glanz, K., \& Bishop, D. B. (2010). The Role of Behavioral Science Theory in Development and Implementation of Public Health Interventions. Annual Review of Public Health, 31(1), 399-418. https://doi.org/10.1146/annurev.publhealth.012809.103604

Godin, G., \& Kok, G. (1996). The theory of planned behavior: a review of its applications to health-related behaviors. American Journal of Health Promotion, 11(2), 87-98.

Goldenberg, J. L., \& Arndt, J. (2008). The implications of death for health: a terror management health model for behavioral health promotion. Psychological Review, 115(4), 1032.

Green, L., Fry, A. F., \& Myerson, J. (1994). Discounting of Delayed Rewards: A Life-Span Comparison. Psychological Science, 5(1), 33-36. https://doi.org/10.1111/j.14679280.1994.tb00610.x

Griskevicius, V., Delton, A. W., Robertson, T. E., \& Tybur, J. M. (2011). Environmental Contingency in Life History Strategies: The Influence of Mortality and Socioeconomic Status on Reproductive Timing. Journal of Personality and Social Psychology, 100(2), 241-254. https://doi.org/Doi 10.1037/A0021082

Griskevicius, V., Tybur, J. M., Delton, A. W., \& Robertson, T. E. (2011). The influence of mortality and socioeconomic status on risk and delayed rewards: A life history theory approach. Journal of Personality and Social Psychology, 100(6), 1015-1026. https://doi.org/10.1037/a0022403

Griskevicius, V., Tybur, J. M., \& Van den Bergh, B. (2010). Going Green to Be Seen: Status, Reputation, and Conspicuous Conservation. Journal of Personality and Social Psychology, 98(3), 392-404. https://doi.org/Doi 10.1037/A0017346

Gruijters, S. L. K. (2016). Baseline comparisons and covariate fishing: Bad statistical habits we should have broken yesterday. European Health Psychologist, 18(5), 205-209.

Gruijters, S. L. K. (2017). The reasoned actions of an espresso machine: a comment on Peters and Crutzen (2017). Health Psychology Review, 11(2), 125-129. https://doi.org/10.1080/17437199.2017.1306716

Gruijters, S. L. K., \& Fleuren, B. P. I. (2017). Measuring the Unmeasurable: The Psychometrics of Life History Strategy. Human Nature, 29(1), 1-12. https://doi.org/10.1007/s12110-017-9307-x 
Gruijters, S. L. K., \& Peters, G.-J. Y. (2017). Quantifying the effectiveness of behavior change interventions. https://doi.org/10.17605/OSF.IO/2BAU7

Gruijters, S. L. K., Tybur, J. M., Massar, K., \& Ruiter, R. A. C. (2018). Function and Fitness: The added value of an evolutionary psychology in explaining health behavior. Manuscript in Preparation.

Gruijters, S. L. K., Tybur, J. M., Ruiter, R. A. C., \& Massar, K. (2016). Sex, germs, and health: pathogen-avoidance motives and health-protective behaviour. Psychology \& Health, 31(8), 959-975. https://doi.org/10.1080/08870446.2016.1161194

Hagger, M. S. (2009). Theoretical integration in health psychology: Unifying ideas and complementary explanations. British Journal of Health Psychology, 14(2), 189-194. https://doi.org/10.1348/135910708X397034

Hagger, M. S., \& Chatzisarantis, N. L. D. (2009). Integrating the Theory of Planned Behaviour and Self-Determination Theory in health behaviour: A meta-analysis. British Journal of Health Psychology, 14, 275-302. https://doi.org/10.1348/135910708X373959

Haig, D. (2013). Proximate and ultimate causes: How come? and what for? Biology and Philosophy, 28(5), 781-786. https://doi.org/10.1007/s10539-013-9369-z

Hamilton, W. (1964). The genetical evolution of social behaviour, I and II. J. Theor. Biol., 7, 1. Hamilton, W. D., Axelrod, R., \& Tanese, R. (1990). Sexual reproduction as an adaptation to resist parasites (a review). Proceedings of the National Academy of Sciences of the United States of America, 87(9), 3566-73. https://doi.org/10.1073/pnas.87.9.3566

Hardy, C. L., \& Van Vugt, M. (2006). Nice Guys Finish First: The Competitive Altruism Hypothesis. Personality and Social Psychology Bulletin, 32(10), 1402-1413. https://doi.org/10.1177/0146167206291006

Harreveld, F., Pligt, J., Vries, N. K., \& Andreas, S. (2000). The structure of attitudes: Attribute importance, accessibility and judgment. British Journal of Social Psychology, 39(3), 363-380. https://doi.org/10.1348/014466600164543

Haselton, M. G., Bryant, G. A., Wilke, A., Frederick, D. A., Galperin, A., Frankenhuis, W. E., $\&$ Moore, T. (2009). Adaptive rationality: An evolutionary perspective on cognitive bias. Social Cognition, 27(5), 733-763.

Haselton, M. G., \& Buss, D. M. (2010). Error management theory and the evolution of misbeliefs. Behavioral and Brain Sciences, 32(6), 522. https://doi.org/10.1017/S0140525X09991440

Haselton, M. G., Buss, D. M., MG, H., DM, B., Haselton, M. G., \& Buss, D. M. (2000). Error management theory: a new perspective on biases in cross-sex mind reading. Journal of Personality and Social Psychology, 78(1), 81. https://doi.org/10.1037//00223514.78.1.81

Hayes, A. F., \& Scharkow, M. (2013). The relative trustworthiness of inferential tests of the indirect effect in statistical mediation analysis: does method really matter?

Psychological Science, 24(10), 1918-1927. https://doi.org/10.1177/0956797613480187

Hill, C. A., \& Preston, L. K. (1996). Individual differences in the experience of sexual motivation: Theory and measurement of dispositional sexual motives. The Journal of Sex Research, 33(1), 27-45. https://doi.org/10.1080/00224499609551812

Hill, E. M., \& Chow, K. (2002). Life-history theory and risky drinking. Addiction, 97(4), 401413. https://doi.org/10.1046/j.1360-0443.2002.00020.x

Hill, E. M., Jenkins, J., \& Farmer, L. (2008). Family unpredictability, future discounting, and risk taking. The Journal of Socio-Economics, 37(4), 1381-1396. https://doi.org/10.1016/j.socec.2006.12.081

Hill, S. E., \& Durante, K. M. (2011). Courtship, Competition, and the Pursuit of Attractiveness: Mating Goals Facilitate Health-Related Risk Taking and Strategic Risk Suppression in Women. Personality and Social Psychology Bulletin, 37(3), 383-394. https://doi.org/10.1177/0146167210395603 
Hill, S. E., Prokosch, M. L., DelPriore, D. J., Griskevicius, V., \& Kramer, A. (2016). Low Childhood Socioeconomic Status Promotes Eating in the Absence of Energy Need. Psychological Science, 27(3), 354-364. https://doi.org/10.1177/0956797615621901

Holbrook, C. (2016). Branches of a twisting tree: Domain-specific threat psychologies derive from shared mechanisms. Current Opinion in Psychology. https://doi.org/10.1016/j.copsyc.2015.08.006

Hone, L. S. E., Carter, E. C., \& McCullough, M. E. (2013). Drinking Games as a Venue for Sexual Competition. Evolutionary Psychology, 11(4), 147470491301100. https://doi.org/10.1177/147470491301100413

Hone, L. S. E., \& McCullough, M. (2015). Sexually Selected Sex Differences in Competitiveness Explain Sex Differences in Changes in Drinking Game Participation. Evolutionary Psychology, 13(2), 147470491501300. https://doi.org/10.1177/147470491501300206

Houben, K., Nederkoorn, C., \& Jansen, A. (2014). Eating on impulse: The relation between overweight and food-specific inhibitory control. Obesity, 22(5). https://doi.org/10.1002/oby.20670

Houben, K., Nederkoorn, C., Wiers, R. W., \& Jansen, A. (2011). Resisting temptation: Decreasing alcohol-related affect and drinking behavior by training response inhibition. Drug and Alcohol Dependence, 116(1-3), 132-136. https://doi.org/10.1016/j.drugalcdep.2010.12.011

Hu, L. T., \& Bentler, P. M. (1999). Cutoff criteria for fit indexes in covariance structure analysis: Conventional criteria versus new alternatives. Structural Equation Modeling: A Multidisciplinary Journal, 6(1), 1-55. https://doi.org/Doi $10.1080 / 10705519909540118$

Janz, N. K., \& Becker, M. H. (1984). The health belief model: A decade later. Health Education \& Behavior, 11(1), 1-47.

Jha, P., Ramasundarahettige, C., Landsman, V., Rostron, B., Thun, M., Anderson, R. N., ... Peto, R. (2013). 21st-Century Hazards of Smoking and Benefits of Cessation in the United States. New England Journal of Medicine, 368(4), 341-350. https://doi.org/10.1056/NEJMsa1211128

Joffe, G. P., Foxman, B., Schmidt, A. J., Farris, K. B., Carter, R. J., Neumann, S., ... Walters, A. M. (1992). Multiple partners and partner choice as risk factors for sexually transmitted disease among female college students. Sex Transm Dis, 19(5), 272-278.

Joreskog, K. G., \& Goldberger, A. S. (1975). Estimation of a Model with Multiple Indicators and Multiple Causes of a Single Latent Variable. Journal of the American Statistical Association, 70(351), 631-639. https://doi.org/10.2307/2285946

Kaplan, H. S., \& Gangestad, S. W. (2005). Life history theory and evolutionary psychology. The Handbook of Evolutionary Psychology, 2(3), 68-95. https://doi.org/10.1002/evan.1360020303

Kelly, S., \& Dunbar, R. I. M. (2001). Who dares, wins. Human Nature, 12(2), 89-105. https://doi.org/10.1007/s12110-001-1018-6

Kenrick, D. T., Griskevicius, V., Neuberg, S. L., \& Schaller, M. (2010). Renovating the pyramid of needs contemporary extensions built upon ancient foundations. Perspectives on Psychological Science, 5(3), 292-314. https://doi.org/Doi $10.1177 / 1745691610369469$

Kenrick, D. T., Neuberg, S. L., Griskevicius, V., Becker, D. V, \& Schaller, M. (2010). GoalDriven Cognition and Functional Behavior: The Fundamental-Motives Framework. Current Directions in Psychological Science, 19(1), 63-67. https://doi.org/Doi $10.1177 / 0963721409359281$

Kirkpatrick, L. A., \& Navarrete, C. D. (2006). Reports of my death anxiety have been greatly exaggerated: A critique of terror management theory from an evolutionary perspective. Psychological Inquiry, 17(4), 288-298. https://doi.org/10.1080/10478400701366969 
Kreager, D. A., Staff, J., Gauthier, R., Lefkowitz, E. S., \& Feinberg, M. E. (2016). The Double Standard at Sexual Debut: Gender, Sexual Behavior and Adolescent Peer Acceptance. Sex Roles, 75(7-8), 377-392. https://doi.org/10.1007/s11199-016-0618-x

Kurzban, R., Burton-Chellew, M. N., \& West, S. A. (2015). The Evolution of Altruism in Humans. Annual Review of Psychology, 66(1), 575-599. https://doi.org/10.1146/annurev-psych-010814-015355

Kurzban, R., Dukes, A., \& Weeden, J. (2010). Sex, drugs and moral goals: reproductive strategies and views about recreational drugs. Proceedings of the Royal Society B: Biological Sciences, 277(1699), 3501-3508. https://doi.org/10.1098/rspb.2010.0608

Kurzban, R., \& Leary, M. R. (2001). Evolutionary origins of stigmatisation: the functions of social exclusion. Psychological Bulletin, 127(2), ? https://doi.org/10.1037//00332909.127.2.187

Kurzban, R., Tooby, J., \& Cosmides, L. (2001). Can race be erased? Coalitional computation and social categorization. Proceedings of the National Academy of Sciences, 98(26), 15387-15392. https://doi.org/10.1073/pnas.251541498

Laland, K. N., Sterelny, K., Odling-Smee, J., Hoppitt, W., \& Uller, T. (2011). Cause and Effect in Biology Revisited: Is Mayr's Proximate-Ultimate Dichotomy Still Useful? Science, 334(6062), 1512-1516. https://doi.org/10.1126/science.1210879

Landau, M. J., Solomon, S., Pyszczynski, T., \& Greenberg, J. (2007). On the compatibility of terror management theory and perspectives on human evolution. Evolutionary Psychology, 5(3), 476-519. https://doi.org/10.1177/147470490700500303

Lieberman, D. L., Tybur, J. M., \& Latner, J. D. (2012). Disgust Sensitivity, Obesity Stigma, and Gender: Contamination Psychology Predicts Weight Bias for Women, Not Men. Obesity, 20(9), 1803-1814. https://doi.org/10.1038/oby.2011.247

Lim, S. S., Vos, T., Flaxman, A. D., Danaei, G., Shibuya, K., Adair-Rohani, H., ... Ezzati, M. (2012). A comparative risk assessment of burden of disease and injury attributable to 67 risk factors and risk factor clusters in 21 regions, 1990-2010: a systematic analysis for the Global Burden of Disease Study 2010. The Lancet, 380(9859), 2224-2260. https://doi.org/10.1016/S0140-6736(12)61766-8

Lindberg, C., Lewis-Spruill, C., \& Crownover, R. (2006). Barriers to sexual and reproductive health care: urban male adolescents speak out. Issues in Comprehensive Pediatric Nursing, 29(2), 73-88 16p. Retrieved from http:/ / search.ebscohost.com/login.aspx?direct=true\&db=rzh\&AN=106310999\&site=e host-live

Lindell, M. K., \& Whitney, D. J. (2001). Accounting for common method variance in crosssectional research designs. Journal of Applied Psychology, 86(1), 114-121. https://doi.org/10.1037/0021-9010.86.1.114

Lippke, S., Nigg, C. R., \& Maddock, J. E. (2012). Health-promoting and health-risk behaviors: Theory-driven analyses of multiple health behavior change in three international samples. International Journal of Behavioral Medicine, 19(1), 1-13. https://doi.org/10.1007/s12529-010-9135-4

Lorenc, T., Marrero-Guillamon, I., Aggleton, P., Cooper, C., Llewellyn, A., Lehmann, A., \& Lindsay, C. (2011). Promoting the uptake of HIV testing among men who have sex with men: systematic review of effectiveness and cost-effectiveness. Sexually Transmitted Infections, 87(4), 272-278. https://doi.org/10.1136/sti.2010.048280

MacCallum, R. C., \& Austin, J. T. (2000). Applications of structural equation modeling in psychological research. Annual Review of Psychology, 51, 201-226. https://doi.org/10.1146/annurev.psych.51.1.201

Maestripieri, D., \& Roney, J. R. (2006). Evolutionary developmental psychology: Contributions from comparative research with nonhuman primates. Developmental Review, 26(2), 120-137.

Malek, A. M. (2013). Delay in Seeking Care for Sexually Transmitted Diseases in Young Men and Women Attending a Public STD Clinic. The Open AIDS Journal, 7(1), 7-13. https://doi.org/10.2174/1874613620130614002 
Maner, J. K., Gailliot, M. T., Rouby, D. A., \& Miller, S. L. (2007). Can't take my eyes off you: Attentional adhesion to mates and rivals. Journal of Personality and Social Psychology, 93(3), 389-401. https://doi.org/10.1037/0022-3514.93.3.389

Massar, K., \& Buunk, A. P. (2009). Rivals in the mind's eye: Jealous responses after subliminal exposure to body shapes. Personality and Individual Differences, 46(2), 129134. https://doi.org/10.1016/j.paid.2008.09.016

Mayr, E. (1961). Cause and Effect in Biology: Kinds of causes, predictability, and teleology are viewed by a practicing biologist. Science, 134(3489), 1501-1506. https://doi.org/10.1126/science.134.3489.1501

McAloney, K., Graham, H., Law, C., \& Platt, L. (2013). A scoping review of statistical approaches to the analysis of multiple health-related behaviours. Preventive Medicine, 56(6), 365-371. https://doi.org/10.1016/j.ypmed.2013.03.002

Meertens, R., Branković, I., Ruiter, R. A. C., Lohstroh, E., Schaalma, H. P., Brankovic, I., ... Schaalma, H. P. (2013). Dirty love: the effect of cleanliness of the environment on perceived susceptibility for sexually transmitted infections. Journal of Applied Social Psychology, 43(S1), E56-E63. https://doi.org/Doi 10.1111/Jasp.12051

Méjean, C., Droomers, M., van der Schouw, Y. T., Sluijs, I., Czernichow, S., Grobbee, D. E., ... Beulens, J. W. J. (2013). The contribution of diet and lifestyle to socioeconomic inequalities in cardiovascular morbidity and mortality. International Journal of Cardiology, 168(6), 5190-5195. https://doi.org/10.1016/j.ijcard.2013.07.188

Mevissen, F. E. F., Ruiter, R. a C., Meertens, R. M., Zimbile, F., \& Schaalma, H. P. (2011). Justify your love: testing an online STI-risk communication intervention designed to promote condom use and STI-testing. Psychology \& Health, 26(2), 205-21. https://doi.org/10.1080/08870446.2011.531575

Michie, S., \& Johnston, M. (2012). Theories and techniques of behaviour change: Developing a cumulative science of behaviour change. Health Psychology Review, 6(1), 1-6. https://doi.org/10.1080/17437199.2012.654964

Michie, S., Johnston, M., Francis, J., Hardeman, W., \& Eccles, M. (2008). From Theory to Intervention: Mapping Theoretically Derived Behavioural Determinants to Behaviour Change Techniques. Applied Psychology, 57(4), 660-680. https://doi.org/10.1111/j.1464-0597.2008.00341.x

Morris, J. L., Lippman, S. A., Philip, S., Bernstein, K., Neilands, T. B., \& Lightfoot, M. (2014). Sexually Transmitted Infection Related Stigma and Shame Among African American Male Youth: Implications for Testing Practices, Partner Notification, and Treatment. AIDS Patient Care and STDs, 28(9), 499-506. https://doi.org/10.1089/apc.2013.0316

Murray, D. R., Jones, D. N., \& Schaller, M. (2013). Perceived threat of infectious disease and its implications for sexual attitudes. Personality and Individual Differences, 54(1), 103-108. https://doi.org/10.1016/j.paid.2012.08.021

Navarrete, C. D., \& Fessler, D. M. T. (2006). Disease avoidance and ethnocentrism: the effects of disease vulnerability and disgust sensitivity on intergroup attitudes. Evolution and Human Behavior, 27(4), 270-282. https://doi.org/10.1016/j.evolhumbehav.2005.12.001

Nederkoorn, C., Smulders, F. T. Y., Havermans, R. C., Roefs, A., \& Jansen, A. (2006). Impulsivity in obese women. Appetite, 47(2), 253-256. https://doi.org/10.1016/j.appet.2006.05.008

Nesse, R. M. (2005). Natural selection and the regulation of defenses: A signal detection analysis of the smoke detector principle. Evolution and Human Behavior, 26(1), 88-105. https://doi.org/DOI 10.1016/j.evolhumbehav.2004.08.002

Nettle, D. (2006). The evolution of personality variation in humans and other animals. American Psychologist, 61(6), 622-631. https://doi.org/10.1037/0003-066X.61.6.622

Nettle, D. (2009a). Evolution and Genetics for Psychology. New York, US.: Oxford University Press.

Nettle, D. (2009b). Social class through the evolutionary lens. Psychologist, 22(11), 934-937. 
Nettle, D. (2010a). Dying young and living fast: variation in life history across English neighborhoods. Behavioral Ecology, 21(2), 387-395.

https://doi.org/10.1093/beheco/arp202

Nettle, D. (2010b). Why Are There Social Gradients in Preventative Health Behavior? A Perspective from Behavioral Ecology. PLoS ONE, 5(10), e13371. https://doi.org/10.1371/journal.pone.0013371

Nettle, D., Andrews, C., \& Bateson, M. (2016). Food insecurity as a driver of obesity in humans: The insurance hypothesis. Behavioral and Brain Sciences, 1-34. https://doi.org/10.1017/S0140525X16000947

Nettle, D., \& Bateson, M. (2015). Adaptive developmental plasticity: what is it, how can we recognize it and when can it evolve? Proc $R$ Soc B, 282(1812), 20151005-. https://doi.org/10.1098/rspb.2015.1005

Nettle, D., Frankenhuis, W. ., \& Rickard, I. J. (2013). The evolution of predictive adaptive responses in human life history. Proc Biol Sci, 280(1766), 20131343. https://doi.org/10.1098/rspb.2013.1343

Nettle, D., Gibson, M. A., Lawson, D. W., \& Sear, R. (2013). Human behavioral ecology: Current research and future prospects. Behavioral Ecology. https://doi.org/10.1093/beheco/ars222

Neuberg, S. L., Kenrick, D. T., \& Schaller, M. (2010). Evolutionary Social Psychology. In Handbook of Social Psychology. Hoboken, NJ, USA, NJ, USA: John Wiley \& Sons, Inc. https://doi.org/10.1002/9780470561119.socpsy002021

Neuberg, S. L., Kenrick, D. T., \& Schaller, M. (2011). Human threat management systems: Self-protection and disease avoidance. Neuroscience \& Biobehavioral Reviews, 35(4), 1042-1051. https://doi.org/10.1016/j.neubiorev.2010.08.011

Neuberg, S. L., \& Schaller, M. (2016). An evolutionary threat-management approach to prejudices. Current Opinion in Psychology, 7, 1-5. https://doi.org/10.1016/j.copsyc.2015.06.004

Neuberg, S. L., Smith, D. M., \& Asher, T. (2000). Why people stigmatize: Toward a biocultural framework. In T. F. Heatherton, K. R. E., M. R. Hebl, \& J. G. Hull (Eds.), The social psychology of stigma (pp. 31-61). New York: Guilford Press.

Newton, J. D., Ewing, M. T., Burney, S., \& Hay, M. (2012). Resolving the theory of planned behaviour's "expectancy-value muddle" using dimensional salience. Psychology \& Health, 27(5), 588-602. https://doi.org/10.1080/08870446.2011.611244

Newton, J. D., Newton, F. J., \& Ewing, M. T. (2014). The dimensional salience solution to the expectancy-value muddle: An extension. Psychology \& Health, 29(12), 1458-1475. https://doi.org/10.1080/08870446.2014.950657

Noble, N., Paul, C., Turon, H., \& Oldmeadow, C. (2015). Which modifiable health risk behaviours are related? A systematic review of the clustering of Smoking, Nutrition, Alcohol and Physical activity ("SNAP") health risk factors. Preventive Medicine, 81, 1641. https://doi.org/10.1016/j.ypmed.2015.07.003

Nunn, C. L., \& Barton, R. A. (2001). Comparative methods for studying primate adaptation and allometry. Evolutionary Anthropology, 10(3), 81-98. https://doi.org/10.1002/evan.1019

O' Sullivan, B., McGee, H., \& Keegan, O. (2008). Comparing solutions to the expectancyvalue muddle in the theory of planned behaviour. British Journal of Health Psychology, 13(4), 789-802. https://doi.org/10.1348/135910708X278306

Oaten, M. J., Stevenson, R. J., \& Case, T. I. (2009). Disgust as a disease-avoidance mechanism. Psychological Bulletin, 135(2), 303-321. https://doi.org/10.1037/a0014823

Oaten, M. J., Stevenson, R. J., \& Case, T. I. (2011). Disease avoidance as a functional basis for stigmatization. Philosophical Transactions of the Royal Society B: Biological Sciences, 366, 3433-3452. https://doi.org/10.1098/rstb.2011.0095 
Okada, K. (2013). Is omega squared less biased? A comparison of three major effect size indices in one-way ANOVA. Behaviormetrika, 4O(2), 129-147. https://doi.org/10.2333/bhmk.40.129

Olatunji, B. O. (2008). Disgust, scrupulosity and conservative attitudes about sex: Evidence for a mediational model of homophobia. Journal of Research in Personality, 42(5), 1364-1369. https://doi.org/10.1016/j.jrp.2008.04.001

Olatunji, B. O., Adams, T., Ciesielski, B., David, B., Sarawgi, S., \& Broman-Fulks, J. (2012). The Three Domains of Disgust Scale: Factor Structure, Psychometric Properties, and Conceptual Limitations. Assessment, 19(2), 205-225. https://doi.org/10.1177/1073191111432881

Oppenheimer, D. M., Meyvis, T., \& Davidenko, N. (2009). Instructional manipulation checks: Detecting satisficing to increase statistical power. Journal of Experimental Social Psychology, 45(4), 867-872.

https://doi.org/http://dx.doi.org/10.1016/j.jesp.2009.03.009

Park, J. H. (2003). Evolved Disease-Avoidance Processes and Contemporary Anti-Social Behavior: Prejudicial Attitudes and Avoidance of People with Physical Disabilitiestle. Journal of Nonverbal Behavior, 27(2), 65-87. https://doi.org/10.1023/A:1023910408854

Park, J. H., Schaller, M., \& Crandall, C. S. (2007). Pathogen-avoidance mechanisms and the stigmatization of obese people. Evolution and Human Behavior, 28(6), 410-414. https://doi.org/10.1016/j.evolhumbehav.2007.05.008

Parsons, J. T., Siegel, A. W., \& Cousins, J. H. (1997). Late adolescent risk-taking: Effects of perceived benefits and perceived risks on behavioral intentions and behavioral change. Journal of Adolescence, 20(4), 381-392.

Patrick, M. E., Maggs, J. L., Cooper, M. L., \& Lee, C. M. (2011). Measurement of Motivations For and Against Sexual Behavior. Assessment, 18(4), 502-516. https://doi.org/10.1177/1073191110372298

Peer, E., Brandimarte, L., Samat, S., \& Acquisti, A. (2017). Beyond the Turk: Alternative platforms for crowdsourcing behavioral research. Journal of Experimental Social Psychology, 70, 153-163. https://doi.org/10.1016/j.jesp.2017.01.006

Penke, L., \& Asendorpf, J. B. (2008). Beyond Global Sociosexual Orientations: A More Differentiated Look at Sociosexuality and Its Effects on Courtship and Romantic Relationships. Journal of Personality and Social Psychology, 95(5), 1113-1135. https://doi.org/Doi 10.1037/0022-3514.95.5.1113

Pepper, G. V., \& Nettle, D. (2014). Socioeconomic disparities in health behaviour: An evolutionary perspective. In Applied Evolutionary Anthropology: Darwinian Approaches to Contemporary World Issues (pp. 225-239). https://doi.org/10.1007/978-1-49390280-4_10

Pepper, G. V, \& Nettle, D. (2017). The behavioural constellation of deprivation: causes and consequences. Behavioral and Brain Sciences, 1-72. https://doi.org/10.1017/S0140525X1600234X

Peters, G.-J. Y. (2014). The alpha and the omega of scale reliability and validity. The European Health Psychologist, 16(2), 56-69.

Peters, G.-J. Y., \& Crutzen, R. (2017). Pragmatic Nihilism: How a Theory of Nothing can Help Health Psychology Progress. Health Psychology Review, 1-39. https://doi.org/10.1080/17437199.2017.1284015

Peters, G.-J. Y., \& Kok, G. (2016). All models are wrong, but some are useful: a comment on Ogden (2016). Health Psychology Review, 10(3), 265-268. https://doi.org/10.1080/17437199.2016.1190658

Peters, G.-J. Y., Ruiter, R. A. C., \& Kok, G. (2013). Threatening communication: a critical re-analysis and a revised meta-analytic test of fear appeal theory. Health Psychology Review, 7(sup1), S8-S31. https://doi.org/10.1080/17437199.2012.703527 
Phelan, J. C., Link, B. G., \& Dovidio, J. F. (2008). Stigma and prejudice: One animal or two? Social Science \& Medicine, 67(3), 358-367.

https://doi.org/10.1016/j.socscimed.2008.03.022

Pietraszewski, D., Cosmides, L., \& Tooby, J. (2014). The Content of Our Cooperation, Not the Color of Our Skin: An Alliance Detection System Regulates Categorization by Coalition and Race, but Not Sex. PLoS ONE, 9(2), e88534. https://doi.org/10.1371/journal.pone.0088534

Pligt, J. van der, \& De Vries, N. K. (1998). Expectancy-Value models of health behaviour: The role of salience and anticipated affect. Psychology \& Health, 13(2), 289-305. https://doi.org/10.1080/08870449808406752

Podsakoff, P. M., MacKenzie, S. B., Lee, J.-Y., \& Podsakoff, N. P. (2003). Common method biases in behavioral research: A critical review of the literature and recommended remedies. Journal of Applied Psychology, 88(5), 879-903.

https://doi.org/10.1037/0021-9010.88.5.879

Pyszczynski, T., Greenberg, J., \& Solomon, S. (1997). Why do we need what we need? A terror management perspective on the roots of human social motivation. Psychological Inquiry, 8(1), 1-20.

Richardson, D., Maple, K., Perry, N., Ambler, E., Jurd, C., \& Fisher, M. (2010). A pilot qualitative analysis of the psychosocial factors which drive young people to decline chlamydia testing in the UK: implications for health promotion and screening. International Journal of STD \& AIDS, 21(3), 187-190. https://doi.org/10.1258/ijsa.2009.009053

Ridley, M. (2004). Evolution (3rd ed.). Oxford, UK. : Blackwell Science Ltd.

Robb, K. A., Simon, A. E., \& Wardle, J. (2009). Socioeconomic Disparities in Optimism and Pessimism. International Journal of Behavioral Medicine, 16(4), 331-338. https://doi.org/10.1007/s12529-008-9018-0

Robbins, R. N., \& Bryan, A. (2004). Relationships Between Future Orientation, Impulsive Sensation Seeking, and Risk Behavior Among Adjudicated Adolescents. Journal of Adolescent Research, 19(4), 428-445. https://doi.org/10.1177/0743558403258860

Rogers, R. W., \& Prentice-Dunn, S. (1997). Protection motivation theory. Handbook of Health Behavior Research 1: Personal and Social Determinants, 113-132. https://doi.org/10.1057/ejis.2009.11

Romer, D. (2010). Adolescent Risk Taking, Impulsivity, and Brain Development: Implications for Prevention. Developmental Psychobiology, 52(3), 263-276. https://doi.org/10.1002/dev.20442

Ronay, R., \& Hippel, W. v. (2010). The Presence of an Attractive Woman Elevates Testosterone and Physical Risk Taking in Young Men. Social Psychological and Personality Science, 1(1), 57-64. https://doi.org/10.1177/1948550609352807

Ronay, R., \& Tybur, J. M. (2017). The wolf will live with the lamb. Behavioral and Brain Sciences, 40, e42. https://doi.org/10.1017/S0140525X16000637

Rosenstock, I. M. (1974). The health belief model and preventive health behavior. Health Education \& Behavior, 2(4), 354-386.

Ryan, R. M., \& Deci, E. L. (2000). Self-determination theory and the facilitation of intrinsic motivation, social development, and well-being. American Psychologist, 55(1), 68-78. https://doi.org/10.1037//0003-066X.55.1.68

Sagebin Bordini, G., \& Sperb, T. M. (2013). Sexual Double Standard: A Review of the Literature Between 2001 and 2010. Sexuality and Culture. https://doi.org/10.1007/s12119-012-9163-0

Sales, J. M., DiClemente, R. J., Rose, E. S., Wingood, G. M., Klein, J. D., \& Woods, E. R. (2007). Relationship of STD-Related Shame and Stigma to Female Adolescents' Condom-Protected Intercourse. Journal of Adolescent Health, 40(6), 573.e1-573.e6. https://doi.org/10.1016/j.jadohealth.2007.01.007 
Schaller, M. (2011). The behavioural immune system and the psychology of human sociality. Philosophical Transactions of the Royal Society B-Biological Sciences, 366(1583), 34183426. https://doi.org/DOI 10.1098/rstb.2011.0029

Schaller, M. (2014). When and how disgust is and is not implicated in the behavioral immune system. Evolutionary Behavioral Sciences, 8(4), 251-256. https://doi.org/10.1037/ebs0000019

Schaller, M., \& Duncan, L. A. (2007). The behavioral immune system: Its evolution and social psychological implications. Evolution and the Social Mind: Evolutionary Psychology and Social Cognition, 293-307.

Schaller, M., Kenrick, D. T., Neel, R., \& Neuberg, S. L. (2017). Evolution and human motivation: A fundamental motives framework. Social and Personality Psychology Compass, 11(6), e12319. https://doi.org/10.1111/spc3.12319

Schaller, M., \& Park, J. H. (2011). The behavioral immune system (and why it matters). Current Directions in Psychological Science, 20(2), 99-103. https://doi.org/10.1177/0963721411402596

Schaller, M., \& Park, J. H. (2011). The Behavioral Immune System (and Why It Matters). Current Directions in Psychological Science, 20(2), 99-103. https://doi.org/10.1177/0963721411402596

Scott-Phillips, T. C., Dickins, T. E., \& West, S. a. (2011). Evolutionary Theory and the Ultimate-Proximate Distinction in the Human Behavioral Sciences. Perspectives on Psychological Science, 6(1), 38-47. https://doi.org/10.1177/1745691610393528

Scott-Sheldon, L. A. J., Huedo-Medina, T. B., Warren, M. R., Johnson, B. T., \& Carey, M. P. (2011). Efficacy of Behavioral Interventions to Increase Condom Use and Reduce Sexually Transmitted Infections. JAIDS Journal of Acquired Immune Deficiency Syndromes, 58(5), 489-498. https://doi.org/10.1097/QAI.0b013e31823554d7

Sheeran, P., Maki, A., Montanaro, E., Avishai-Yitshak, A., Bryan, A., Klein, W. M. P. P., ... Rothman, A. J. (2016). The impact of changing attitudes, norms, and self-efficacy on health-related intentions and behavior: A meta-analysis. Health Psychology, 35(11), 1178-1188. https://doi.org/10.1037/hea0000387

Simpson, J. A., Griskevicius, V., Kuo, S. I., Sung, S., \& Collins, W. A. (2012). Evolution, stress, and sensitive periods: the influence of unpredictability in early versus late childhood on sex and risky behavior. Dev Psychol, 48(3), 674-686. https://doi.org/10.1037/a0027293

Sprengelmeyer, R., Young, A. W., Pundt, I., Sprengelmeyer, A., Calder, A. J., Berrios, G., ... Przuntek, H. (1997). Disgust implicated in obsessive-compulsive disorder. Proceedings of the Royal Society B: Biological Sciences, 264(1389), 1767-1773. https://doi.org/10.1098/rspb.1997.0245

Stearns, S. (1989). Trade-offs in life-history evolution. Functional Ecology, 3(3), 259-268. https://doi.org/10.2307/2389364

Stearns, S. (1992). The evolution of life histories. Oxford: Oxford University Press.

Steinberg, L. (2008). A social neuroscience perspective on adolescent risk-taking. Developmental Review, 28(1), 78-106. https://doi.org/10.1016/j.dr.2007.08.002

Strecher, V. J., Champion, V. L., \& Rosenstock, I. M. (1997). The health belief model and health behavior.

Strecher, V. J., \& Rosenstock, I. M. (1997). The health belief model. Cambridge Handbook of Psychology, Health and Medicine, 113-117.

Stretcher, V., \& Rosenstock, I. M. (1997). The Health Belief Model. Health Behavior and Health Education: Theory, Research and Practice. https://doi.org/10.1111/j.13652648.2010.05450.x

Stringhini, S., Carmeli, C., Jokela, M., Avendaño, M., Muennig, P., Guida, F., ... Zins, M. (2017). Socioeconomic status and the $25 \times 25$ risk factors as determinants of premature mortality: a multicohort study and meta-analysis of 1.7 million men and women. The Lancet, 389(10075), 1229-1237. https://doi.org/10.1016/S01406736(16)32380-7 
Stringhini, S., Sabia, S., Shipley, M., Brunner, E., Nabi, H., Kivimaki, M., \& Singh-Manoux, A. (2010). Association of socioeconomic position with health behaviors and mortality. JAMA : The Journal of the American Medical Association, 303(12), 1159-1166. https://doi.org/10.1001/jama.2010.297

Suls, J., \& Rothman, A. (2004). Evolution of the Biopsychosocial Model: Prospects and Challenges for Health Psychology. Health Psychology, 23(2), 119-125. https://doi.org/10.1037/0278-6133.23.2.119

Taylor, S. E., Fiske, S. T., Etcoff, N. L., \& Ruderman, A. J. (1978). Categorical and contextual bases of person memory and stereotyping. Journal of Personality and Social Psychology, 36(7), 778-793. https://doi.org/10.1037/0022-3514.36.7.778

ten Hoor, G. A., Plasqui, G., Schols, A. M. W. J., \& Kok, G. (2014). Combating adolescent obesity. Current Opinion in Clinical Nutrition and Metabolic Care, 17(6), 521-524. https://doi.org/10.1097/MC0.0000000000000099

Tilson, E. C., Sanchez, V., Ford, C. L., Smurzynski, M., Leone, P. A., Fox, K. K., ... Miller, W. C. (2004). Barriers to asymptomatic screening and other STD services for adolescents and young adults: focus group discussions. BMC Public Health, 4(1), 21. https://doi.org/10.1186/1471-2458-4-21

Tinbergen, N. (1963). On aims and methods of Ethology (as reprinted in Animal Biology, 2005). Zeitschrift Für Tierpsychologie, 20, 410-433.

Tooby, J., \& Cosmides, L. (1990). The past explains the present. Emotional adaptations and the structure of ancestral environments. Ethology and Sociobiology, 11(4-5), 375-424. https://doi.org/10.1016/0162-3095(90)90017-Z

Trafimow, D. (2017). Why I Am Not a Fan of Pragmatic Nihilism. Health Psychology Review, 1-5. https://doi.org/10.1080/17437199.2017.1306717

Turkheimer, E. (2000). Three Laws of Behavior Genetics and What They Mean. Current Directions in Psychological Science, 9(5), 160-164. https://doi.org/10.1111/14678721.00084

Tybur, J. M., Bryan, A. D., \& Hooper, A. E. C. (2012). An evolutionary perspective on health psychology: new approaches and applications. Evolutionary Psychology : An International Journal of Evolutionary Approaches to Psychology and Behavior, 10(5), 855-67. Retrieved from http://www.ncbi.nlm.nih.gov/pubmed/23253791

Tybur, J. M., Bryan, A. D., Magnan, R. E., \& Hooper, A. E. C. (2011). Smells Like Safe Sex: Olfactory Pathogen Primes Increase Intentions to Use Condoms. Psychological Science, 22(4), 478-480. https://doi.org/Doi 10.1177/0956797611400096

Tybur, J. M., \& de Vries, R. E. (2013). Disgust sensitivity and the HEXACO model of personality. Personality and Individual Differences, 55(6), 660-665. https://doi.org/10.1016/j.paid.2013.05.008

Tybur, J. M., Frankenhuis, W. E., \& Pollet, T. V. (2014). Behavioral immune system methods: Surveying the present to shape the future. Evolutionary Behavioral Sciences, 8(4), 274-283. https://doi.org/10.1037/ebs0000017

Tybur, J. M., Inbar, Y., Aarøe, L., Barclay, P., Barlow, F. K., de Barra, M., ... Žeželj, I. (2016). Parasite stress and pathogen avoidance relate to distinct dimensions of political ideology across 30 nations. Proceedings of the National Academy of Sciences, 113(44), 12408-12413. https://doi.org/10.1073/pnas. 1607398113

Tybur, J. M., Inbar, Y., Güler, E., \& Molho, C. (2015). Is the relationship between pathogen avoidance and ideological conservatism explained by sexual strategies? Evolution and Human Behavior, 36(6), 489-497. https://doi.org/10.1016/j.evolhumbehav.2015.01.006

Tybur, J. M., \& Lieberman, D. (2016). Human pathogen avoidance adaptations. Current Opinion in Psychology. https://doi.org/10.1016/j.copsyc.2015.06.005

Tybur, J. M., Lieberman, D., \& Griskevicius, V. (2009). Microbes, mating, and morality: Individual differences in three functional domains of disgust. Journal of Personality and Social Psychology, 97(1), 103-122. https://doi.org/10.1037/a0015474 
Tybur, J. M., Lieberman, D., Kurzban, R., \& DeScioli, P. (2013). Disgust: evolved function and structure. Psychological Review, 120(1), 65-84.

https://doi.org/10.1037/a0030778

Tybur, J. M., Merriman, L. A., Hooper, A. E. C., McDonald, M. M., \& Navarrete, C. D. (2010). Extending the Behavioral Immune System to Political Psychology: Are Political Conservatism and Disgust Sensitivity Really Related? Evolutionary Psychology, 8(4), 147470491000800. https://doi.org/10.1177/147470491000800406

van der Pligt, J., de Vries, N. K., Pligt, J., \& Vries, N. K. (1998). Belief Importance in Expectancy-Value Models of Attitudes1. Journal of Applied Social Psychology, 28(15), 1339-1354. https://doi.org/10.1111/j.1559-1816.1998.tb01680.x

Van Lier, J., Revlin, R., \& De Neys, W. (2013). Detecting Cheaters without Thinking: Testing the Automaticity of the Cheater Detection Module. PLoS ONE, 8(1), e53827. https://doi.org/10.1371/journal.pone.0053827

van Schaik, C. P., Isler, K., \& Burkart, J. M. (2012). Explaining brain size variation: From social to cultural brain. Trends in Cognitive Sciences. https://doi.org/10.1016/j.tics.2012.04.004

van Vugt, M., \& Hardy, C. L. (2009). Cooperation for reputation: Wasteful contributions as costly signals in public goods. Group Processes \& Intergroup Relations, 13(1), 101-111. https://doi.org/10.1177/1368430209342258

Vanneste, S., Verplaetse, J., Verhiel, A., \& Braeckman, J. (2007). Attention bias toward noncooperative people. A dot probe classification study in cheating detection. Evolution and Human Behavior, 28(4), 272-276.

https://doi.org/10.1016/j.evolhumbehav.2007.02.005

Voorspoels, W., Bartlema, A., \& Vanpaemel, W. (2014). Can race really be erased? A preregistered replication study. Frontiers in Psychology, 5. https://doi.org/10.3389/fpsyg.2014.01035

Wang, X. T., Kruger, D. J., \& Wilke, A. (2009). Life history variables and risk-taking propensity. Evolution and Human Behavior, 30(2), 77-84. https://doi.org/10.1016/j.evolhumbehav.2008.09.006

Webb, R. H., \& Wilshire, H. G. (1983). Environmental Effects of Off-Road Vehicles. (R. H. Webb \& H. G. Wilshire, Eds.). New York, NY: Springer New York. https://doi.org/10.1007/978-1-4612-5454-6

Webb, T. L., \& Sheeran, P. (2006). Does changing behavioral intentions engender behavior change? A meta-analysis of the experimental evidence. Psychological Bulletin, 132(2), 249-268. https://doi.org/10.1037/0033-2909.132.2.249

White, A. E., Kenrick, D. T., \& Neuberg, S. L. (2013). Beauty at the Ballot Box: Disease Threats Predict Preferences for Physically Attractive Leaders. Psychological Science. https://doi.org/10.1177/0956797613493642

Wiers, R. W., Rinck, M., Kordts, R., Houben, K., \& Strack, F. (2010). Retraining automatic action-tendencies to approach alcohol in hazardous drinkers. Addiction, 105(2), 279287. https://doi.org/10.1111/j.1360-0443.2009.02775.x

Wilke, A., Hutchinson, J. M. C., Todd, P. M., \& Kruger, D. J. (2006). Is Risk Taking Used as a Cue in Mate Choice? Evolutionary Psychology, 4(1), 147470490600400. https://doi.org/10.1177/147470490600400130

World Health Organization. (2016). Sexually transmitted infections (STIs). Retrieved from http://www.who.int/mediacentre/factsheets/fs 110/en/

Young, S. D., Nussbaum, A. D., \& Monin, B. (2007). Potential Moral Stigma and Reactions to Sexually Transmitted Diseases: Evidence for a Disjunction Fallacy. Personality and Social Psychology Bulletin, 33(6), 789-799. https://doi.org/10.1177/0146167207301027

Zaikman, Y., Marks, M. J., Young, T. M., \& Zeiber, J. A. (2016). Gender role violations and the sexual double standard. Journal of Homosexuality, 1-22. 
SUMMARY 
People often engage knowingly and willingly in behaviors that negatively affect health. Understanding the psychology behind health behaviors - the processes that generate behavior - is one of the main objectives for health psychologists. Understanding these psychological processes is a necessary condition for researchers' ability to craft successful health behavior interventions - and, in turn, understanding is built on a foundation of empirical tests of theory. Hence, one important goal for health psychology is to continuously improve, broaden, and solidify the theory-base used to understand health behaviors. The main focus of this dissertation is to explore whether - and, if so - how an evolutionary perspective can add to theory in health psychology. We do so by applying this perspective foremost to a specific health behavior - screening for sexually-transmitted infections (STI).

In Chapter 2 the evolutionary psychological literature relevant for understanding health behavior is reviewed. The chapter starts with a discussion on, and examples of, the ultimate-proximate distinction used to demarcate evolutionary approaches from the standard psychological disciplines. This chapter further reviews how evolutionary theory may be well-suited to inform researchers about the a priori feasibility of proximate hypotheses, and how it can serve meta-theoretical function for HBT. A key claim made in this chapter is that evolution is unlikely to have equipped human psychology with a motivation - or 'drive', 'instinct'- to be healthy. The chapter further reviews how evolution can help make sense of variation in (and clusters of) unhealthy behaviors resulting from circumstances in early child development.

Chapter 3 describes the results of two cross-sectional empirical studies. The studies examine how trait pathogen-avoidance motivation part of what has been referred to as the behavioral immune system - relate to health-protective behaviors. The studies used self-reported measures of attitude and intention to test for STI, and history of casual sex encounters, as indicators of health-protective behavior in a structural equation model. Data collected in Study 1 revealed that pathogen-avoidance motivation related to participants' attitude and intention towards STI screening. High levels of pathogen-avoidance motivation were also related to having had fewer sexual partners, which partially mediated the effect of pathogenavoidance variables on testing motivation. Study 2 extended these findings by showing moderate associations between pathogen-avoidance motivation and a broad range of health-protective behaviors, including but not limited to pathogen-related health concerns. 
Chapter 4 reports work that addresses how state-activation of pathogen-avoidance relates to health-protective motivation. We hypothesized that temporarily activating a pathogen-avoidance motivation, through eliciting pathogen-disgust, would influence participants' attitude towards STI testing. Given that attitudes are seen to arise from specific behavioral beliefs (the consequences of a behavior), we also examined whether beliefs relating to pathogen-avoidance would more strongly predict attitudes - as compared to motivationally irrelevant beliefs. Results partially supported hypotheses, showing changes in belief importance between-groups. However, our pathogen-avoidance manipulation failed to induce changes in the direct measures of STI testing attitude. We conclude that state-level motivations may contribute to understanding which beliefs shape individuals' attitude towards STI-testing.

In Chapter $\mathbf{5}$ it is examined whether pathogen-avoidance motivations may underlie negativity towards people who get tested for STI. We hypothesized, and compared the relevance of, two such motivations: avoidance of infectious and promiscuous individuals. By designing vignettes describing various different individuals, this chapter explored whether merely getting tested for STI already increases negativity towards those getting tested, and - if so - which motivation relates to negative attitudes and attributed shame most clearly. Study $1 \quad(N=245)$ found positive associations between perceptions of a target's infectiousness and promiscuity and negative attitudes and attributed shame toward that target - but contrary to expectation, STD-testing decreased perceived infectiousness and promiscuity. Additionally, STD-testing by the target decreased participants' negative attitudes and attributed shame. Study 2 $(N=793)$ replicated these effects by experimentally manipulating information regarding target infectiousness and promiscuity. We conclude that STDtesting does not increase perceptions of infectiousness and promiscuity, but that these variables are independent sources of negative attitudes and attributed shame. 

SAMENVATTING 
Mensen vertonen willens en wetens gedragingen die de gezondheid beschadigen. Het begrijpen van de psychologie achter gezondheidsgedrag, de processen die het gedrag sturen, is een van de hoofddoelstellingen voor gezondheidspsychologen. Om succesvolle interventies op het gebied van gezondheidsgedrag te kunnen creëren is het begrijpen van deze psychologische processen een noodzakelijke voorwaarde. Dit begrip is gebaseerd op een basis van empirische toetsing van theoretische voorspellingen. Een belangrijk doel voor de gezondheidspsychologie is daarom om de theoretische kern van de discipline te verbeteren, te verbreden en te versterken. Het hoofddoel van dit proefschrift is om te onderzoeken of en hoe een evolutionair psychologisch perspectief kan bijdragen aan de theoretische fundamenten van de gezondheidspsychologie. In deze dissertatie doen we dat door dit evolutionair psychologische perspectief toe te passen op een specifiek gezondheidsgedrag: de motivatie van individuen om te testen voor seksueel overdraagbare infecties (SOA).

In hoofdstuk 2 wordt evolutionair-psychologische literatuur die relevant is voor het begrijpen van gezondheidsgedrag besproken en geëvalueerd. Verder staat in dit hoofdstuk de vraag wat een evolutionair perspectief kan bijdragen aan het begrip van gezondheidsgedrag centraal. Het hoofdstuk begint met een discussie over (en voorbeelden van) het zogenaamde 'ultimaat-proximaat' onderscheid; waarmee evolutionaire benaderingen zich afbakenen van de standaard psychologische disciplines. In dit hoofdstuk wordt verder besproken hoe geschikt de evolutietheorie is om onderzoekers te informeren over de redelijkheid van theoretische voorspellingen. Bovendien bespreken we hoe de evolutionaire psychologie een meta-theoretische functie voor gezondheidspsychologische theorie kan hebben. Een belangrijke bewering in dit hoofdstuk is dat evolutie waarschijnlijk niet geleid heeft tot een menselijke motivatie - of 'drive', of 'instinct', om gezond te zijn. In dit hoofdstuk wordt verder betoogd dat evolutietheorie kan bijdragen aan het begrip van variatie in (en clusters van) ongezond gedrag. Hier ligt de nadruk op de omstandigheden in de vroege ontwikkeling van een kind en hoe deze op een functioneel adaptieve manier de volwassen psyche vormgeven.

Hoofdstuk 3 beschrijft de resultaten van twee cross-sectionele empirische studies. De studies onderzoeken hoe een infectievermijdingsmotivatie relateert aan gezondheid beschermend gedrag. De studies gebruikten zelfrapportage maten, met betrekking tot attitude en intentie om te testen voor SOA, en geschiedenis van losse seksuele contacten, als indicatoren van gezondheid beschermend gedrag. Data verzameld in Studie 1 onthulden dat infectie-vermijdingsmotivatie relateert aan de attitude en de intentie van deelnemers om te testen op SOA. Hogere niveaus van infectie-vermijdingsmotivatie waren ook gerelateerd aan het hebben van minder losse seksuele partners. De hoeveelheid losse seksuele partners medieerde het effect van infectie-vermijdingsvariabelen op attitude en intentie ook gedeeltelijk. In Studie 2 werden deze bevindingen 
gerepliceerd, maar ook uitgebreid door het vaststellen van associaties tussen infectie-vermijdingsmotivering en een breed scala aan gezondheid beschermende gedragingen (zoals de intentie om een vreemd uitziende moedervlek te laten onderzoeken).

Hoofdstuk 4 rapporteert werk dat onderzocht hoe tijdelijke activatie van infectie-vermijdingmotieven gezondheid beschermende motivatie beïnvloedt. We verwachtten dat tijdelijk activatie van een infectie-vermijdingsmotivatie (door middel van het induceren van walging), invloed op de attitudes ten aanzien van SOA testen zou hebben. Gezien het feit dat attitudes verondersteld worden te ontstaan op basis van specifieke overtuigingen, hebben we ook bekeken of bepaalde overtuigingen belangrijkere voorspellers van attitude werden dan andere door de walgingsmanipulatie. De resultaten ondersteunden de hypothesen gedeeltelijk. Hoewel de walgingsmanipulatie geen directe invloed had op de attitudes van proefpersonen, vonden wij wel dat specifieke overtuigingen belangrijkere voorspellers van attitude werden. We sluiten dit hoofdstuk af met de suggestie dat overtuigingen op flexibele wijze vormgeven aan attitudes van mensen, ofwel, dat het belang van bepaalde ideeën, kennis en overtuigingen afhangt van de motivationele toestand waarin mensen zich bevinden.

In hoofdstuk 5 wordt onderzocht of infectie-vermijdingsmotivatie een onderliggende oorzaak is van negatieve houdingen naar mensen die zich laten testen op SOA. We onderzochten of mensen die zich laten testen op SOA als een bedreiging gezien worden: ofwel omdat testers gezien worden als besmettelijk, ofwel omdat zij gezien worden als promiscue. Door het ontwerpen van zogenaamde vignetten (hypothetische beschrijvingen van personen), wordt in dit hoofdstuk bekeken of alleen al het gaan testen op SOA, de negatieve houdingen ten opzichte van degenen die zich laat testen verhoogt. Ook bekijken we welke motivatie (vermijden van infecties, of vermijden van seksueel losbandige individuen) ten grondslag ligt aan die negatieve houdingen. Studie 1 vond positieve associaties tussen percepties van het besmettingsrisico van een persoon en promiscuiteit enerzijds en negatieve beoordelingen ten opzichte van de omschreven persoon anderzijds. Een onverwachte vondst was dat mensen die zich laten testen op SOA, als minder besmettelijk en promiscue worden beoordeeld. Daarnaast verminderden SOAtesten de negatieve attitudes van deelnemers en toegeschreven schaamte. In Studie 2 hebben we de informatie m.b.t. besmettelijkheid en promiscuïteit experimenteel gemanipuleerd, en werden de resultaten uit Studie 1 gerepliceerd. Op basis van deze studies concluderen we dat een SOA-test niet de percepties van besmettelijkheid en promiscuïteit versterkt, maar dat deze variabelen onafhankelijke bronnen van negatieve houdingen zijn. Het proefschrift concludeert met de stelling dat de evolutionaire psychologie een nieuw perspectief op gezondheidsgedrag kan bieden, en mogelijk een bron voor nieuwe hypotheses over gezondheidsgedrag. 

VALORIzation 
Science is - for a large part - about moving beyond observable facts. To achieve an understanding of phenomena, a common method for scientists is to let go of what is apparent and to find underlying mechanisms or 'hidden essences' - as such, it involves 'carving nature at its joints' (cf. Phaedrus, Plato). Physicists attempt to explain to behavior of middle-sized objects by delving deeper and deeper into the structure of atoms (e.g. strings). Biologists try to understand disease by carving a way into the structure of cells, up until the DNA. And similarly, psychologists try to explain behavior by unravelling the structure and architecture of the human mind - its biological basis (e.g. neurons) or its cognitive design underlying psychological determinants of behavior (e.g. people's desires, beliefs and attitudes). Thus, often, these kinds of reductionist efforts to understanding the non-obvious, hidden, mechanisms that could illuminate why things happen, inherently involves a move towards a theoretical realm. This makes much of science by definition an anti-practical undertaking. The realm of scientific explanation is often very much detached from the things society cares about. For example, how does understanding how attitudes are formed help us address a societal problem? Why is an understanding of particular psychological mechanisms relevant to society? This is the question of valorization, and answering it requires us to inflate research results from the realm of abstract theoretical terms to the level of observable effects.

Describing the relevance and societal importance of scientific findings is fraught with difficulty. For one, a priori, relevance as it is apparent now can be very much detached from the description of relevance given to it $a$ posteriori - time must tell. Historically, this is illustrated by the discovery of DNA (Watson \& Crick, 1953) - the ultimate valorization of this discovery lies in discoveries decades later (including understanding and screening for genetic disease) and more valorization is probably yet to come. And second, it is often a subjective matter whether an invention is relevant or of value. But for this dissertation, valorization was embedded in the goal of the project, so I will attempt to lay-out some key points. I will focus on the relevance of the project - as I see it.

In this dissertation, we have tried to examine how evolutionary psychology can help understand health behavior. This project was abstract in the sense that integration of two scientific disciplines - evolutionary psychology and health psychology - was the broader project goal. A more distal goal, however, is that this integration is attempted to expand the theoretical foundations of health psychology, which in turn should lead to an increased understanding of health behavior. We did so by focusing on one health behavior in particular- peoples' attitudes towards and intention to test for sexually-transmitted infections (STI). Infectious disease pose challenges to health and well-being. Despite the many efforts to promote safe sexual practices (see Scott-Sheldon et al., 2011), STI are prevalent and a source of health problems (World Health Organization, 2016). Available 
treatments for STI (either curative or palliative) can have no effect when people remain undiagnosed - therefore, actively seeking health care services and getting screened are critical steps needed to be taken by individuals. The relevance of trying to understand how people's inclination to test for STI arises is clear: testing for STI is a societal relevant and beneficial behavior as it has the potential to decrease the disease burden caused by these infections. The societal burden caused by disease is twofold. First, disease caused by STI can decrease people's quality of life which is a cost to society as a whole. Second, decreasing disease prevalence also decreases an economic burden on society.

If we know the relevant processes, variables and triggers of healthprotective behaviors, this knowledge can be applied to benefit health promotion campaigns. As part of our evolutionary approach, we have looked at processes that we believe are part of human's "instinctive", evolved health psychology - as opposed to things that we learn to stay away from. An important component of our evolved health psychology, we proposed, may include pathogen-disgust- and we found some evidence that this emotion relates to differences in health behavior. This knowledge is potentially relevant in that it could mean that for example health promotion campaigns, aiming to increase testing behavior, could leverage this specific emotion to promote testing. Additionally, it may find application outside the domain of STD in promoting various other health behaviors. There is no good reason that the aspects of our evolved health psychology we examined (i.e. pathogen-avoidance) do not have the potential to improve societal health behavior in other domains - example in designing effective persuasive communication methods to warn people about the risk of particular behavior (e.g. smoking, drinking, etc.). Indeed, results reported in Chapter 3 of this dissertation indicate that pathogen-avoidance motivation (e.g. pathogen disgust) is likely related to a broad array of health-protective behaviors. A question that needs to be further examined, though, is how health promotion programs can best utilize this motivational system in order to promote healthy behavior. Some findings reported in this dissertation, hopefully, provide a foundation for future research which may translate to health psychologists' ability to promote health behavior, and perhaps eventually to decrease the societal burden causes by disease. 

Curriculum Vitae 
Stefan L.K. Gruijters was born on September 13, 1986, in Geleen, a small city in the southern province of Limburg the Netherlands. In 1999 he attended high school at Graaf Huyn College, of which he dropped out in the $4^{\text {th }}$ grade. After a brief intermezzo, which included cooking school and fulltime bar tending, Stefan pursued adult education and received a higher secondary education degree in 2006. After a move to the city of Tilburg in 2006, he obtained higher-education propaedeutics in applied psychology in 2007. Since 2007, Stefan has been living in Maastricht, where he received a bachelor's degree in cognitive psychology, and a master's degree in developmental psychology - both obtained at Maastricht University. After his master's studies he briefly worked as a research and teaching assistant at the Department of Work and Social Psychology, where he started a PhD project. In addition to working on his $\mathrm{PhD}$-project, he developed and taught bachelor courses in the psychology program, and published several methodological projects. Currently, Stefan is employed as a lecturer at the Open University of the Netherlands in Heerlen. 
Publication list 


\section{$\underline{\text { Published }}$}

Gruijters, S.L.K., \& Fleuren, B.P.I. (2018). Measuring the Unmeasurable: The psychometrics of life history strategy. Human Nature, 29 (1), 1-12.

Gruijters, S.L.K. (2017). The reasoned actions of an espresso machine: A comment on Peters \& Crutzen (2017). Health Psychology Review, 11 (2), 121-125.

Gruijters, S.L.K. (2017). Waarom we niet wegrennen voor giftige paddenstoelen [Why we don't flee from poisonous mushrooms]. De Psycholoog, 52 (7), 32-39.

Gruijters, S.L.K. (2016). Baseline comparisons and covariate fishing: Bad statistical habits we should have broken yesterday. European Health Psychologist, 18(5), 205-209.

Gruijters, S. L. K., Tybur, J. M., Ruiter, R. A. C., \& Massar, K. (2016). Sex, germs, and health: pathogen-avoidance motives and health-protective behaviour. Psychology \& Health, 31(8), 959-975.

Massar, K., Buunk, A. P., \& Gruijters, S. L. K. (2013). Pregnant Women's View on Their Relationship: A Comparison With Nonpregnant Women. Interpersona, 7(2), 272.

\section{Under Review}

Gruijters, S.L.K., Tybur, J. M., Massar,K., \& Ruiter, R.A.C. (under review). What is negative about testing for sexually-transmitted infections?

Gruijters, S.L.K., Ruiter, R. A. C., Massar, K., \& Tybur, J. M. (under review). Attitude in a motivational context: The effect of elicited pathogenavoidance motivation on screening attitude and belief importance.

Gruijters, S.L.K., \& Peters, G-J.Y. (revising). Quantifying the effectiveness of behavior change interventions: Introduction to a practical measure of effect size.

\section{Unpublished Work}

Gruijters, S.L.K., Tybur, J. M., Massar, K., \& Ruiter, R. A. C. (2018). Function and fitness: The added value of an evolutionary psychological perspective in explaining health behavior. Manuscript in preparation.

Gruijters, S.L.K., Massar, K., Pletzers, J. \& Kok, G. (2013). Effectevaluatie 'Can You Fix It?' Eindrapport. Work \& Social Psychology. Universiteit Maastricht. 


\section{$\underline{\text { Presentations }}$}

Gruijters, S.L.K, Tybur, J. M., Ruiter, R. A. C., \& Massar, K. (2017). Attributed shame and negative attitudes towards STI-testers: A threat management perspective. Poster presented at the European Health Psychology Society conference 2017.

Gruijters, S.L.K \& Peters, G-J.Y. (2017) Introducing the Numbers Needed for Change (NNC): An effect size that connects research to practice. Talk presented at the European Health Psychology Society conference 2017.

Peters, G-J. Y., Crutzen, R., Gruijters, S.L.K. (2017). Sample sizes required for successful intervention research. Talk presented at the European Health Psychology Society conference 2017.

Gruijters, S.L.K, Tybur, J. M., Ruiter, R. A. C., \& Massar, K. Pathogens, sex and health. Talk presented at the annual meeting of the Human Behavior \& Evolution Society, Vancouver, Canada (2016).

Gruijters, S.L.K, Tybur, J. M., Ruiter, R. A. C., \& Massar, K. Sex, Germs and health. Talk presented at the annual research day of Maastricht University. (2015). 

ACKNOWLEDGEMENTS 
Completing a PhD-project is an emotional, cognitive, and sometimes a physical marathon - initially fueled by intrinsic motivations and ideals, but inevitably requiring the occasional sunk-cost-effect top-ups. Along PhD-way, however, a lot of academic gasoline is also provided by the people you work with, family, and friends. This is the place where I would like to thank some of these people for doing so.

To my supervision team in Maastricht (Rob and Karlijn), thank you for giving me the chance to start a $\mathrm{PhD}$, and for investing your time and effort in supervising me over these past four years. Both of you have made a considerable effort to get this project going, and I'm very thankful for that. Josh - although we have perhaps seen too little of each other during the project, thank you for always joining arms with Rob and Karlijn in providing me guidance from a distance. I want to add some special thanks - Josh - for the extensive and consistent comments on my English. Your papers set an example of fine academic prose, and I'm fortunate to have had your guidance in writing.

I would like to mention some people that did not contribute to the completion of this dissertation - Arie, Gjalt-Jorn, Rob dV, Harry, and Michael. You have all been mentors to me in activities and topics that hugely distracted me from doing actual empirical research (as one should), but are perhaps so much more fun to do - Teaching, Philosophy, Theory, and Methods (or, TPTM, as an abbreviation-fancying academic would put it). Arie, you asked me to be a tutor in your course when I was still a bachelor student, later supervised my master thesis - an internship that almost literally ended with me in the gutter - gave me the opportunity to give my first lecture, and so on. These experiences were decisive in setting me on an academic path towards both teaching and research - and I'm thankful for that, and all the guidance and support you have given me over these last years. Gjalt-Jorn, thanks for fueling and supporting my enthusiasm in methodology and for being a scientific mentor. Rob dV, thank you for 'philosophically ruining me as a scientist', as you once put it, by introducing me to philosophy of mind. Harry, you have mentored me in evolution theory and introduced me (i.e. gave me regular mini-lectures) to interesting issues and ideas in evolutionary biology / genetics - a baggage I'm very grateful to carry with me. Michael thank you for the support and putting trust in my teaching abilities.

My paranympho's. Bram (Pronounced - Brèm), it is hard to put a number on how many hours we have bounced ideas of each other with regard to teaching, talked about evolution, and brainstormed about methodology. The - intellectual - stimulation and personal support you provided as an office mate, on an almost daily basis, have been critical to me in finishing this project. And Mart, you have been a late but very pleasant addition to our office. Thank you for often brightening the mood in the office with your many fun stories, and also for your support during my last year of the project. 
To my WSP colleagues, there are too many names to mention, but thank you for all the enjoyable lunch conversations and the many laughs. Gerjo, thank you for always helping me to find nuance in situations. Fred, thank you for minding my office hygiene. A long-gone colleague, Birthe, thanks for all the nice memories, and the many fun music and beer nights in Maastricht. Joa, thanks for getting me, and keeping me for a while, in good physical shape over these last years - and Tobias thanks for countering these efforts with the regular BBQs. To Ellen, thanks for the supporting talks and chats - often outside in the cold. Also, my new colleagues at OU, Jannes in particular, thanks for the warm and supportive welcome. And Alicia, thanks for all the good talks and fun times, I'm happy that I get to share rides with one of my favorite colleagues to the new work place - I hope many good talks on the way are yet to come.

Tot slot, dudes. Ilja, Niek, Thijs, Tjibbe, Mike - dank voor de regelmatige luchtige verzetjes en vermaeck.

Mijn ouders, voor wie dit proefschrift er vooral ligt, zelfs nadat ik een niveautje lager werd gezet, het blijven zitten in de 4de klas, het volledig stoppen met de middelbare school in de $4^{\text {de }}$ klas, het gerook, gedrink en geruzie, leken jullie er eigenlijk nog wel vertrouwen in te houden. Bijzonder. Dank voor de niet minder wordende steun over de jaren heen. De broers en schoonzussen, ook jullie dank voor de steun tijdens de vele downs de afgelopen jaren.

And finally, Rachel, who has been a tower of strength, or - as we say in Dutch - my rock in the surf. It helps so much to have you around. 
